\title{
Kinetic Inference Resolves Epigenetic Mechanism of Drug Resistance in Melanoma
}

Yapeng Su, ${ }^{1,2,3,14}$ Xiang Lu, ${ }^{4,14}$ Guideng Li, ${ }^{3}$ Chunmei Liu, ${ }^{1}$ Yan Kong, ${ }^{5}$ Jihoon W. Lee, ${ }^{2}$ Rachel $\mathrm{Ng},{ }^{2}$ Stephanie Wong, ${ }^{3}$ Lidia Robert, ${ }^{6}$ Charles Warden, ${ }^{7}$ Victoria Liu, ${ }^{2}$ Jie Chen, ${ }^{8}$ Zhuo Wang, ${ }^{8}$ Yezi Yang, ${ }^{4}$ Hanjun Cheng, ${ }^{1}$ Alphonsus H. C. Ng, ${ }^{1}$ Guangrong Qin, ${ }^{1}$ Songming Peng, ${ }^{2}$ Min Xue, ${ }^{2}$ Dazy Johnson, ${ }^{4}$ Yu Xu, ${ }^{9}$ Jinhui Wang, ${ }^{7}$ Xiwei Wu, ${ }^{7}$ Ilya Shmulevich, ${ }^{1}$ Qihui Shi, ${ }^{8,10}$ Raphael Levine, ${ }^{4,11}$ Antoni Ribas, ${ }^{4,6,12,13}$ David Baltimore, ${ }^{3}$ Jun Guo, ${ }^{5,15}$ James R. Heath, ${ }^{1,2,15, *}$ and Wei Wei ${ }^{1,4,13,15,16, *}$

${ }^{1}$ Institute for Systems Biology, Seattle, WA 98109, USA

${ }^{2}$ Division of Chemistry and Chemical Engineering, California Institute of Technology, Pasadena CA 91125, USA

${ }^{3}$ Division of Biology and Biological Engineering, California Institute of Technology, Pasadena CA 91125, USA

${ }^{4}$ Department of Molecular and Medical Pharmacology, University of California - Los Angeles, Los Angeles CA 90095, USA

${ }^{5}$ The Key Laboratory of Carcinogenesis and Translational Research (Ministry of Education), Department of Renal Cancer and Melanoma, Peking University Cancer Hospital and Institute, Beijing, China

${ }^{6}$ Department of Medicine, University of California - Los Angeles, Los Angeles CA 90095, USA

${ }^{7}$ Department of Molecular and Cellular Biology, City of Hope, Duarte, CA 91010, USA

${ }^{8}$ Key Laboratory of Systems Biomedicine (Ministry of Education), Shanghai Center for Systems Biomedicine, Shanghai Jiao Tong University, Shanghai, China.

${ }^{9}$ Fudan University Shanghai Cancer Center, Shanghai, China

${ }^{10}$ Key Laboratory of Medical Epigenetics and Metabolism, Institute of Biomedical Sciences, Fudan University, Shanghai, China

${ }^{11}$ The Fritz Haber Research Center, The Hebrew University, Jerusalem, Israel

${ }^{12}$ Department of Surgery, University of California - Los Angeles, Los Angeles, CA 90095, USA

${ }^{13}$ Jonsson Comprehensive Cancer Center, University of California - Los Angeles, Los Angeles, CA 90095, USA

${ }^{14}$ The authors contributed equally

${ }^{15}$ Senior author

${ }^{16}$ Lead Contact

*Correspondence: jheath@systemsbiology.org (J.R.H.), wwei@ systemsbiology.org (W.W.) 


\section{Summary}

We resolved a mechanism connecting tumor epigenetic plasticity with non-genetic adaptive resistance to therapy, with MAPK inhibition of $B R A F$-mutant melanomas providing the model. These cancer cells undergo multiple, reversible drug-induced cell-state transitions, ultimately yielding a drug-resistant mesenchymal-like phenotype. A kinetic series of transcriptome and epigenome data, collected over two months of drug treatment and release, revealed changing levels of thousands of genes and extensive chromatin remodeling. However, a 3-step computational algorithm greatly simplified the interpretation of these changes, and revealed that the whole adaptive process was controlled by a gene module activated within just three days of treatment, with RelA driving chromatin remodeling to establish an epigenetic program encoding long-term phenotype changes. These findings were confirmed across several patient-derived cell lines and in melanoma patients under MAPK inhibitor treatment. Co-targeting BRAF and histone-modifying enzymes arrests adaptive transitions towards drug tolerance in epigenetically plastic melanoma cells and may be exploited therapeutically. 


\section{Introduction}

The acquisition of therapy resistance in cancer patients remains a major clinical challenge (Arkenau et al., 2011; Brock et al., 2009; Pritchard et al., 2012; Salgia and Kulkarni, 2018). While various genetic mutations have been reported to cause resistance (Nazarian et al., 2010; Wagle et al., 2011), recent literature also points to the importance of epigenetic modulations to drugtolerance that can precede the emergence of drug-resistant genotypes in a variety of tumor types (Bai et al., 2019; Hata et al., 2016; Hugo et al., 2015; Pisco et al., 2013; Rambow et al., 2018; Ramirez et al., 2016; Sharma et al., 2010; Wang et al., 2018). For such epigenetic processes, tumor cells adapt to the drug treatment by orchestrating master transcription factors and chromatin remodelers within a regulatory network. The resultant changes of chromatin profile via histone modifications eventually establish specific gene expression programs of the drug-tolerant state (Brown et al., 2014; Dawson and Kouzarides, 2012; Fang et al., 2016; Flavahan et al., 2017; Kelly et al., 2010; Knoechel et al., 2014; Liau et al., 2017; Shaffer et al., 2017; Strub et al., 2018; Suvà et al., 2014). Unlike genetic mechanisms, epigenetic cell-state transitions can be reversed upon drug removal (Sharma et al., 2010; Su et al., 2017; Sun et al., 2014). Indeed, such reversibility has been observed in in vitro and in vivo tumor models (Roesch et al., 2010; Sharma et al., 2010; Su et al., 2017; Sun et al., 2014; Das Thakur et al., 2013) and increasingly reported in clinical settings (Cara and Tannock, 2001; Kurata et al., 2004; Lee, 2012; Mackiewicz-Wysocka et al., 2014). Nevertheless, the mechanistic details of these epigenetic modulations remain incompletely understood, thus limiting the options for therapeutic interventions designed to arrest the nongenetic resistance.

Accumulating evidence suggests that phenotypic plasticity is an essential characteristic associated with non-genetic resistance (Flavahan et al., 2017; Hoek et al., 2008; Kemper et al., 2014; Landsberg et al., 2012). Phenotypic plasticity, defined as the ability of cells to reside in distinct phenotypes and switch between them without genomic alterations, is an intrinsic property of cells to survive stressful conditions. Cancer cells can also exploit plasticity to survive drug treatment by transitioning from a drug-sensitive phenotype to drug-tolerant phenotypes (Flavahan et al., 2017; Kochanowski et al., 2018). Paradigmatic examples include certain BRAF-mutant melanomas under MAPK pathway inhibitor (MAPKi) treatment. The drug-naïve melanoma cells initially reside as drug-sensitive melanocytic phenotypes MITF $^{\text {high }}$ and elevated pigmentation genes). Upon continuous MAPK inhibition, they can evolve into a transient, slow-cycling, neuralcrest-like phenotype (MITF ${ }^{\text {low }} / \mathrm{NGFR}^{\text {high }}$ ) (Fallahi-Sichani et al., 2017; Su et al., 2017) and eventually towards a mesenchymal phenotype $\left(\mathrm{MITF}^{\text {low }}, \mathrm{SOX} 10^{\text {low }}\right.$, and elevated mesenchymal markers) (Kemper et al., 2014; Müller et al., 2014; Su et al., 2017; Tsoi et al., 2018). The mesenchymal phenotype is notorious for its resistance to MAPKi as well as many other treatment regimens including immunotherapy (Hugo et al., 2015, 2016; Ramsdale et al., 2015). 
Several studies have explored the molecular markers associated with the drug-tolerant or drugresistant phenotypes including: down-regulation of SOX10 (Shaffer et al., 2017; Sun et al., 2014) and upregulation of JNK/c-JUN (Fallahi-Sichani et al., 2015, 2017; Ramsdale et al., 2015) in the mesenchymal phenotype, as well as upregulation of KDM5 (Roesch et al., 2010, 2013) in a slowcycling (likely neural crest) drug-tolerant phenotype. However, there remains a clear unmet need to identify the early-stage adaptive processes that are triggered immediately following the drug exposure to lead the transition towards drug-tolerant phenotypes. Such an understanding may unveil the molecular nature of phenotypic plasticity and compelling drug targets that can arrest the entire adaptive resistance process prior to the establishment of resistant phenotypes.

We sought to resolve the early-acting regulatory process of adaptive resistance through kinetic characterizations of the transcriptome and functional epigenome of patient-derived $B R A F$-mutant melanoma cell lines featuring varying degrees of phenotypic plasticity. Systems-level analysis of the transition dynamics, followed by experimental validations, discerned the critical transcription factors and chromatin remodelers within a regulatory network that initiated and drove the adaptive cell state transition. The mechanism informed the design of combination therapies to disrupt the chromatin remodeling and to arrest the adaptive transition at a very early stage. The phenotypic plasticity was found to correlate with the efficacy of the drug combinations across multiple melanoma cell lines, implicating that the plasticity may be epigenetically encoded in the baseline chromatin profiles. The signatures of the adaptive mechanism were also found in sequential patient biopsies. Together, our study resolved an early-acting epigenetic mechanism of non-genetic resistance, which may be exploited to prevent targeted therapy resistance in melanoma.

\section{Results}

\section{Reversibility of the adaptive transition in patient-derived $B R A F$-mutant melanoma cell lines}

We used an epigenetically plastic $B R A F^{\mathrm{V} 600 \mathrm{E}}$-mutant melanoma cell line $\mathrm{M} 397$ as a model system to interrogate the kinetics of the adaptive cell state transition in response to continuous BRAF inhibition. We treated the cells with a BRAF inhibitor (BRAFi) for a month, then separated them into two sets: one with an additional month of continuous drug treatment, and the other untreated for one month (Figure 1A). Cells were collected for a time-series transcriptome and functional characterization (Figures 1 and S1; Table S1). The resultant gene expression data after 29 days (D29) of drug exposure showed significant enrichments of mesenchymal signatures, cellular invasiveness, migration, and loss of MITF targets (Figures 1C and S1D; Table S2). The adapted cells were slow-cycling, as evidenced by reduced proportion of cells in the S and G2/M phases (Figure 1E). An additional month of drug exposure maintained the cells in a steady state with a relatively stable transcriptome profile (Figures 1B and S1C). Drug removal (DR) triggered a reversion to a state with a transcriptome profile that resembled the untreated (D0) state (Figures 1B, S1C and S1D), as illustrated by the fact that the molecular signature enrichments of D29 vs. D0 and DR30 vs. D29 were essentially mirror images of each other (Figure 1C). Furthermore, the reverted cells recovered their proliferative and cell cycle characteristics, and were re-sensitized to 
BRAF inhibition (Figures 1D-1F). These results suggest a fully reversible adaptive transition at the transcriptomic, phenotypic, and functional levels. We further confirmed the existence of such reversibility, at the functional level, in other $B R A F$-mutant melanoma cell lines with varying sensitivities to BRAFi (Figures 1D, 1F, S1A, and S1D). Therefore, the BRAFi-induced adaptive resistance is reversible at both transcriptomic and functional levels with generality across a panel of patient-derived $B R A F$-mutant melanoma cell lines.

\section{Information theoretic analysis of the transcriptome kinetics resolved two gene modules associated with the reversible transition}

To extract the underlying regulatory modules that change coordinately in the reversible transition, we applied information theory-based surprisal analysis to the time-course transcriptome data (Eq. 1). Surprisal analysis was initially formulated to understand the dynamics of nonequilibrium systems (Levine, 1978). It has been extended, in multiple publications (Kravchenko-Balasha et al., 2012, 2014, 2016b, 2016a; Poovathingal et al., 2016; Remacle et al., 2010; Zadran et al., 2013, 2014), to characterize biological processes in living cells. It approximates quantum state distributions of molecular species within a cell's molecular ensemble in order to assess the maximum entropy of those biomolecules. Particularly, for a system characterized by a kinetic series of transcriptome, Equation 1 from surprisal analysis can deconvolute the changes of thousands of genes into one unchanged gene expression baseline and a series of gene expression modules. Each module contains a group of genes that are coordinately changing together across time points (an example gene list for module-1 is visualized in dashedline, circled regions in Figure 2A). We applied this analysis, and then used the resulting gene modules to computationally estimate and visualize cell-state transition trajectories (STAR Methods).

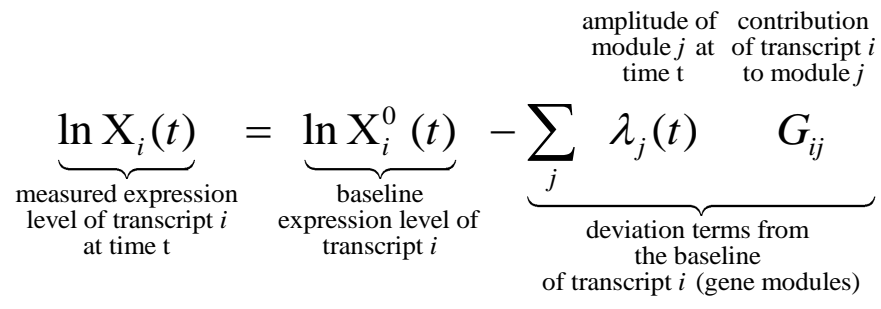

Specifically, in Equation 1, $\ln \mathrm{X}_{i}(t)$, the natural logarithm of the measured level of transcript $i$ at time $t$, is defined as the expression baseline of transcript $i\left(\ln X_{i}^{0}\right)$, minus the sum of gene module alterations weighted by the relative contribution to each module by transcript $i$ $\left(\sum_{j} \lambda_{j}(t) G_{i j}\right.$ ). Each gene module is represented by a time-dependent module amplitude (or score $\left.\lambda_{j}(t)\right)$ that denotes the importance of the gene module $j$ to the global transcriptome at time $t$. Module-specific contribution scores from each transcript $G_{i j}$ denote weight of gene $i$ on module $j$. Thus, the biological functions of each module $j$ can be inferred from genes with higher positive or 
negative module-specific contribution scores $G_{i j}$. The gene expression baseline is the reference point for the entire transition.

The application of Eq. 1 to the time-series of transcriptome data de-convoluted them into two time-dependent gene modules plus the gene expression baseline. To visualize the kinetic changes of the entire transcriptome and each gene module, we plotted the time-series transcriptome profiles and de-convoluted gene modules as self-organizing mosaic maps (SOMs) (Figures 2A and S2A). The gene expression baseline calculated from the surprisal analysis (top row of SOMs in Figures $2 \mathrm{~A}$ and S2A) shows no time dependence. This demonstrates the validity of surprisal analysis in analyzing this system (Remacle et al., 2010). The second and third rows are plots of the two resolved, time-varying gene modules. The SOMs for D0 and DR30 appear nearly identical, reflecting the reversibility of the adaptive response at the transcriptome level as well as at the resolved gene module level. When the baseline state and the two regulatory gene modules are summed (the row labeled 'sum' in Figures 2A and S2A), the resultant SOMs from surprisal analysis closely matched the experimental transcriptome data (bottom row of Figures 2A and S2A). Thus, the expression change of thousands of genes during the reversible transition can be delineated by a time-invariant expression baseline, plus the changes of two time-varying gene modules.

The reduction of the transcriptome kinetic series into two gene modules enables visualization of drug-adaptation trajectories taken by the cells. This is achieved by projecting the time-series transcriptome onto the 2-D cell-state space defined by the gene modules, with each axis representing the module score of each gene module. The plot is a cyclic loop (Figure 2B) comprised of a forward trajectory (blue), and a drug-removal, reverse trajectory (green), which indicates that the cells undertake a different return path to the original drug-sensitive state. This cyclic shape suggests that the two gene modules operate sequentially. The first module, Mearly, was fully activated within the first 3 days of drug treatment (y-axis of Figure 2B), while the second module, $\mathrm{M}_{\text {late, }}$ (x-axis of Figure 2B) gradually activated between days 3 and 29 (D3 and D29). Continued treatment beyond D29 caused minimal change in either module (blue dash line circled region of Figure 2B), in agreement with the stable transcriptome profile observed from D29 to D59 (Figures 1B and S2A). Interestingly, upon drug removal, there was an immediate reduction in the first module, followed by a gradual reversion of the second module to its original pretreatment module score. The different operational dynamics of these modules resulted in the cyclic transition trajectories that the cells took.

To further investigate the biological meaning of the two gene modules, we conducted gene set enrichment analysis (GSEA) on genes ranked by their module-specific contribution scores $\left(G_{i j}\right)$. $\mathrm{M}_{\text {late }}$ was positively associated with melanocytic signatures (e.g. MITF targets) and negatively correlated with mesenchymal signatures, cell invasiveness, and NFKB, TGF $\beta$, and JNK signaling pathways (Figure 2B; Table S3). Consequently, the gradual change of the $\mathrm{M}_{\text {late }}$ score from positive to negative values between D3 and D29 indicates that the drug-treated cells de-differentiated towards neural-crest and mesenchymal phenotypes, with loss of melanocytic signatures, and an 
increase of NFKB, TGF $\beta$, and JNK signaling. Similarly, $\mathrm{M}_{\text {early }}$ was positively associated with HDAC1 activity and negatively associated with cell cycle regulation (Figure 2B), suggesting that the initial drug exposure led to an immediate histone deacetylation and cell cycle arrest. Therefore, the two gene modules resolved from surprisal analysis delineated the stepwise, reversible dynamic changes of cellular functions during the cyclic transitions associated with adaptive resistance development.

\section{Dynamic system modeling discerned the regulatory relationship between the early- and late- gene modules}

A possible implication of the sequential operation of $\mathbf{M}_{\text {early }}$ and $\mathbf{M}_{\text {late }}$ is that the biological processes associated with the two modules are coupled where the completion of the Mearly gene program triggers the expression of $\mathrm{M}_{\text {late }}$ genes. This implies a co-dependency of these two modules. We tested this hypothesis by fitting the dynamic dependence of the two modules to a coarsegrained model resembling a simple two-gene feedback circuit (Eq. 2). This approximation yields an estimate for how the two gene modules are coupled (Figure 2C; see STAR Methods).

$$
\begin{aligned}
& \frac{d\left[\text { gene }_{\mathrm{M}_{\text {early }}}\right]}{d t}=B_{e}+M_{e-e}\left[\text { gene }_{\mathrm{M}_{\text {early }}}\right]+M_{l-e}\left[\text { gene }_{\mathrm{M}_{\text {late }}}\right] \\
& \frac{d\left[\text { gene }_{\mathrm{M}_{\text {late }}}\right]}{d t}=B_{l}+M_{e-l}\left[\text { gene }_{\mathrm{M}_{\text {early }}}\right]+M_{l-l}\left[\text { gene }_{\mathrm{M}_{\text {late }}}\right]
\end{aligned}
$$

Here, the brackets denote the averaged expression level of module-associated genes at a given time point. $B_{e}$ and $B_{l}$ represent the basal production of $\mathrm{M}_{\text {early- }}$ and $\mathrm{M}_{\text {late-associated genes, }}$ respectively. Coefficients $M_{e-e}$ and $M_{l-l}$ reflect self-regulation of $\mathrm{M}_{\text {early }}$ and the $\mathrm{M}_{\text {late }}$ expression, respectively, while coefficients $M_{l-e}$ and $M_{e-l}$ reflect the $\mathrm{M}_{\text {late }}$ regulation over the expression of Mearly genes and vice versa.

The ODE fitting of gene expression associated with $\mathrm{M}_{\text {late }}$ and $\mathrm{M}_{\text {early }}$ for both forward and reverse trajectories revealed that $\mathbf{M}_{\text {early }}$ exerted significant control over both itself and $\mathrm{M}_{\text {late. }}$ This is evidenced by the significantly larger pre-factors $M_{e-e}$ and $M_{e-l}$ (Figures 2D and S3; Table S4). By contrast, $M_{\text {late }}$ yielded only minimal influence over itself or $M_{\text {early. The strong influence of }}$ $\mathrm{M}_{\text {early }}$ applied to both the drug-treated forward and drug-release reverse trajectories (Figures 2D, 2E, S2B and S3). In other words, $\mathrm{M}_{\text {early }}$ dominated the entirety of the forward D0-D29 transition path towards drug resistance as well as the drug removal trajectory back to the drug-sensitive state. Further, the dependence of $\mathbf{M}_{\text {late }}$ on $\mathbf{M}_{\text {early }}$ suggested an orchestrated process whereby certain important transcription factors associated with Mearly played a key role in regulating downstream genes associated with $\mathrm{M}_{\text {late, }}$ ultimately driving the adaptive transition towards the drug resistant phenotype. Thus, dynamic system modeling revealed the strong influence of the early-acting gene module on the late-acting gene module, and implied that $\mathbf{M}_{\text {early }}$ may contain the key regulators that initiated the adaptive cell state transition. 


\section{Inference of critical early-acting upstream transcriptional regulators based upon the inter- dependence of the two gene modules}

Guided by the importance of $\mathrm{M}_{\text {early }}$ module in driving the adaptive resistance (Figure 2D), we hypothesized that certain key transcription factors (TFs) or co-factors within $\mathrm{M}_{\text {early }}$ regulate the downstream genes within $\mathrm{M}_{\text {late }}$ and thus drive the adaptive transition. To test this hypothesis, we used two complementary approaches to infer the early-acting TFs in M397. For the first approach (Figures 3A and S4A), we hypothesized that functionally relevant TFs associated with Mearly should have their target effector genes enriched in the gene set associated with the subsequent action of $\mathrm{M}_{\text {late }}$. Therefore, we first filtered out all the possible TFs and co-factors within $\mathrm{M}_{\text {early }}$ and then acquired their downstream targets from the TF targets database, followed by assessing their enrichment in the genes associated with $\mathrm{M}_{\text {late }}$ (STAR Methods). This identified that subset of

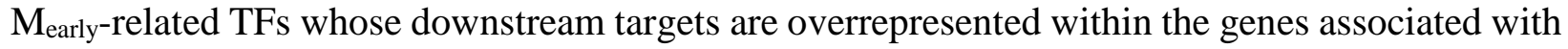
$\mathrm{M}_{\text {late }}$ module. Five statistically enriched TFs and co-factors were identified, with Pearson correlation coefficients $\rho>0.9$ (Figures 3B, panel i). These include MEIS3, which is required for neural-crest invasion (Uribe and Bronner, 2015), NKX3-2, which mediates the epithelialmesenchymal transition in neural crest development (Lim and Thiery, 2012), and LEF1 whose down-regulation is related to non-genomic MAPKi resistance in melanomas (Hugo et al., 2015). These enriched TFs may regulate the cancer cell phenotype changes associated with the forward and reverse transitions (Figure 3C). Most interestingly, the histone modifying enzyme KDM5B (H3K4 demethylase), whose expression displayed a sharp increase by D3, was also found to have many target genes overrepresented in $\mathrm{M}_{\text {late }}$ (Figure 3D). Importantly, this histone modifier has been previously associated with reversible drug-tolerant states in several tumor types, including melanomas (Hinohara et al., 2018; Roesch et al., 2010, 2013).

For the second inference approach, we performed enrichment analysis of cis-regulatory elements in the promoter regions of all genes strongly associated with $\mathrm{M}_{\text {late }}$ (Figure $\mathrm{S} 4 \mathrm{~B}$ ). We ranked these elements according to their statistical significance (Figure 3B, panel ii). The top one ranked element was the binding motif of the NFKB family member RelA, which was recently identified as an important regulator associated with this adaptive transition ( $\mathrm{Su}$ et al., 2017). The expression kinetics of RelA was not associated with either $\mathbf{M}_{\text {late }}$ or $\mathbf{M}_{\text {early. }}$ Instead, the expression level of RelA gradually increased from the start of BRAF inhibition to D29, implying its consistent activity over the entire adaptive transition towards the mesenchymal state (Figure 3D). Interestingly, NFKBIE, which is highly anti-correlated with $\mathrm{M}_{\text {early }}(\rho=-0.88)$ and represses NFKB activation by preventing RelA nuclear translocation, displayed a sharp drop by D3 (Figure 3D). This suggests that activation of RelA and associated downstream genes in $\mathrm{M}_{\text {late }}$ might be mediated by the immediate down-regulation of NFKBIE, thus releasing RelA into the nucleus. A second transcription regulator similarly identified was AP- $2 \alpha$, which has been reported to be involved in melanoma progression and metastasis (Berger et al., 2005). Taken together, these analyses greatly simplified the interpretation of the kinetic transcriptome data by inferring a few controlling, earlyacting TFs and co-factors (Figures 3B and S4B), including RelA and KDM5B, from the large 
numbers of transcripts altered during the course of BRAF inhibition and drug release (Figures S5A and S5B).

\section{Reversibility of chromatin accessibility and histone modification profiles shed light on downstream transcription factors associated with the adaptive transition}

Information theory analysis and dynamic ODE modeling of transcriptome kinetics pinpointed a few key early-acting TFs that likely trigger the initiation of the cell state transition towards resistance. To obtain a complete mechanistic picture, we seek to further identify the late-acting driver regulators that are the downstream targets of those early-acting TFs via cellular epigenome characterization at different stages of the reversible transition (Figure 5A).

Since the previous inference and enrichment analyses pointed to the fast activation of histone modifiers KDM5B and HDAC1 in Mearly that represses activation histone marks (Figures $2 \mathrm{~B}$ and 3B), we first accessed the regulatory regions associated with open chromatin. We accomplished this via transposase-accessible chromatin with high-throughput sequencing (ATAC-seq) and chromatin immunoprecipitation sequencing (ChIP-seq) against two activation histone marks, H3K4me3 and H3K27ac, which are targets of KDM5B and HDAC1. Similar to the transcriptome profiles, the overall chromatin accessibility displayed reversible changes during drug treatment and removal. That accessibility gradually decreased following BRAFi exposure, but after a month of drug removal reverted to a profile similar to that of untreated cells (Figures 4A, 4B and S5C). Overall modification levels of the two histone marks displayed the same reversibility (Figure S5D and S5E). However, the drop in these marks after 3-day BRAFi exposure was large relative to the corresponding small reduction of the ATAC-seq signal (Figures 4A and S5D), suggesting that these histone modifications may precede and perhaps drive the changes in chromatin accessibility. Further, the promoter region of many well-reported regulators for the adaptive resistance in melanoma also displayed reversible changes that aligned well with their reversible expression patterns (Figure S5E). We also tabulated the numbers of differential peaks of H3K4me3 and $\mathrm{H} 3 \mathrm{~K} 27 \mathrm{ac}$ that changed between day 0 and subsequent time points (Figure 4C). The differences between states at long-term drug removal (DR30) and at day 0 were minimal. This was especially true for $\mathrm{H} 3 \mathrm{~K} 27 \mathrm{ac}$, where only two peaks reflected acetylation differences between day 0 and longterm drug removal. These data demonstrated the genome-scale chromatin landscape underwent reversible changes upon drug treatment and removal, thus supporting a potential epigenetic mechanism of the reversible adaptive transition.

To search for causal TFs driving the dynamic changes of the chromatin landscape, we used K-means clustering to analyze the genome-wide chromatin restructuring at four time points spread across the reversible transition (Figure 4B). We identified four clusters of chromatin accessibility peaks with unique kinetics, plus a fifth, time-invariant cluster. For these four clusters, we mined the underlying DNA sequences and searched for over-represented TF binding motifs. The highly enriched motifs in the reversible transition (right side of Figure 4B) contain binding motifs of certain TFs reported previously to be involved in the adaptive resistance of melanomas, including 
MITF (Sáez-Ayala et al., 2013), SOX10 (Sun et al., 2014), Jun-AP1 (Fallahi-Sichani et al., 2015, 2017; Ramsdale et al., 2015), and RelA (Su et al., 2017). Some of these TFs, such as RelA and AP-2, were overlapping with those inferred from the transcriptome data (Figure S4B). To further resolve whether these modifications were modulated by RelA whose motif is ranked top one in the common cis-regulatory element inference (Figures 3B and S4B), we quantified the H3K4me3 and H3K27ac ChIP-seq signals across all the RelA binding sites and found marked reduction after 3 days of drug exposure, with recovery upon drug removal (Figure 4D). It suggests that RelA binds primarily to distal sequences containing both activation histone marks $\mathrm{H} 3 \mathrm{~K} 4 \mathrm{me} 3$ and $\mathrm{H} 3 \mathrm{~K} 27 \mathrm{ac}$, and might regulate them through interactions with the KDM5B and HDAC1 during the adaptive transition.

In addition to confirming RelA as a critical early-acting regulator that may cause the epigenome changes, we further mined downstream regulators that may be the direct targets of RelA and showed consistent epigenome alteration patterns at the RelA binding region across the reversible transition. We achieved this by quantifying the changes in chromatin accessibility and two activation histone marks of all TFs/co-factors associated with $\mathrm{M}_{\text {late }}$ that contain RelA binding motifs (STAR Methods). SOX10 was identified to display the most significant changes across all three epigenome alterations at the RelA binding region (Table S5). Consider the importance of SOX10 in regulating mesenchymal phenotype in melanoma (Shaffer et al., 2017; Sun et al., 2014; Verfaillie et al., 2015), we hypothesized that SOX10 is likely one key downstream regulator for the cell state transition towards resistance. Taken together, these data illustrated the reversibility of adaptive resistance at the level of the global chromatin landscape. The kinetics of the reversible epigenome profiles further pointed to a collection of early-acting and downstream-effector TFs, particularly RelA and SOX10, in regulating such adaptive epigenetic resistance.

\section{Mechanistic regulatory network of adaptive resistance}

Based on the transcriptional regulators inferred from gene module interactions and epigenome profiling, we tied these inferences together with prior knowledge and formed a mechanistic regulatory network (Figure 5A). We hypothesized that for drug-naïve cells, NFKBIE and SOX10 are both epigenetically activated and that downstream TGF $\beta$ signaling is repressed (Sun et al., 2014). BRAF inhibition triggers a sharp drop in NFKBIE expression and a sharp increase in the expression of the histone demethylase KDM5B (Figure 3D). The reduction of NFKBIE would promote the nuclear translocation of RelA (Whiteside, 1997). In the nucleus, RelA would then recruit KDM5B and HDAC1 to repress SOX10 and NFKBIE expression by erasing the activation histone marks in their promoter regions, consistent with the rapid decrease of activation histone marks at RelA binding sites (Figures 4D). The downregulation of SOX10 expression has been reported to promote BRAFi adaptive resistance through promoting the up-regulation of TGF $\beta$ signaling and mesenchymal transition (Figure 3C) (Shaffer et al., 2017; Sun et al., 2014). Our mechanistic hypothesis provides a rationale for how this happens, and further indicates how the downregulation of NFKBIE promotes RelA nuclear translocation, thus establishing a positive feedback loop (Figure 5A). Drug removal reverses this process, starting with the gradual recovery 
of SOX10 expression (Figure 3D), the loss of mesenchymal signatures (Figure 3C), and the eventual re-opening of the chromatin (Figure 4A). We extensively tested this mechanism in the following ways.

We first examined the change in overall chromatin accessibility and levels of the histone marks $\mathrm{H} 3 \mathrm{~K} 4 \mathrm{me} 3$ and $\mathrm{H} 3 \mathrm{~K} 27 \mathrm{ac}$ at the promoter regions of SOX1O and NFKBIE over the course of the adaptive transition. We found reduction of the overall chromatin accessibility and of the levels of both histone marks upon BRAF inhibition, and a recovery of these signals upon drug removal (Figure 5B). These observations confirmed the involvement of chromatin alterations in the gene expression changes of SOX10 and NFKBIE. We next explored the role of RelA in recruiting histone remodelers. We tested whether RelA, KDM5B and HDAC1 simultaneously bind to the promoter regions of SOX10 and NFKBIE. We performed ChIP-PCR experiments on untreated cells using primers targeting the promoter regions of $S O X 10$ and NFKBIE. The results confirmed the co-localization of RelA, KDM5B, HDAC1, and the two histone marks (Figure 5C). Quantitative assessment of binding profiles via ChIP-qPCR further revealed that BRAFi treatment elevated binding of RelA, KDM5B, and HDAC1 to the SOX1O and NFKBIE promoter sites and consequently diminished $\mathrm{H} 3 \mathrm{~K} 4 \mathrm{me} 3$ and $\mathrm{H} 3 \mathrm{~K} 27 \mathrm{ac}$ histone marks (Figure 5D). These binding enrichment profiles reverted to the levels of the untreated cells after drug removal (Figure 5D). Co-immunoprecipitation (Co-IP) assays confirmed the binding of RelA to KDM5B and HDAC1 (Figure 5E), suggesting that RelA can form a complex with either KDM5B or HDAC1. These findings support the role of RelA in recruiting histone remodelers in regulating the expression of SOX10 and NFKBIE.

To further validate that SOX10 and NFKBIE are directly repressed by the recruitment of KDM5B and HDAC1 through RelA, we sought to perturb RelA with a drug that inhibits its nuclear translocation. According to our hypothesis, such inhibition should decrease the recruitment of the histone-modifying enzymes KDMB5 and HDAC1, which, in turn, will increase the H3K4me3 and $\mathrm{H} 3 \mathrm{~K} 27 \mathrm{ac}$ levels at the promoter regions of SOX10 and NFKBIE. We first treated the M397 cells with BRAFi for 21 days to induce adaptive resistance with reduced SOX10 expression (Figure 5F). Under continued BRAFi treatment, we added a drug (JSH-23) to inhibit RelA nuclear translocation (Shin et al., 2004), and monitored short-term interval changes in SOX10 expression. SOX10 expression rapidly increased following JSH-23 treatment and returned to the level originally observed at D0 after 24 hours of drug exposure (Figure 5F). Furthermore, we observed barely detectable binding of RelA, KDM5B, and HDAC1 and consequently increased binding of $\mathrm{H} 3 \mathrm{~K} 4 \mathrm{me} 3$ and H3K23ac at the SOX10 and NFKBIE promoter regions after 24 hours of JSH-23 treatment (Figure 5G). The immediate increase of SOX10 expression and decrease of HDAC1 and KDM5B enrichment within 24 hours of JSH-23 exposure further validated our hypothesis that the repression of SOX10 and NFKBIE is directly through RelA-mediated epigenetic silencing.

We performed additional independent perturbations to validate the proposed molecular mechanism using genetically engineered M397 cells. We first triggered RelA nuclear translocation through CRISPR knockout (KO) of NFKBIE to release the cytoplasmic retention of RelA 
(Spiecker et al., 2000). According to our proposed mechanism, this translocation should in turn enhance recruitment of the histone modifying enzymes HDAC1 and KDM5B, and thus diminish levels of activation histone marks $\mathrm{H} 3 \mathrm{~K} 4 \mathrm{me} 3$ and $\mathrm{H} 3 \mathrm{~K} 27 \mathrm{ac}$ at the promoter regions of SOX10. This prediction was confirmed by ChIP-qPCR result (Figure 5H). The actual expression of SOX10 was consequently reduced in NFKBIE-KO cells relative to control cells (Figure 5I). Similarly, knockout of KDM5B partially upregulated H3K4me3 levels at the SOX10 and NFKBIE promoters (Figure 5J), thus increasing SOX10 and NFKBIE expression (Figure 5K). In addition, we also observed elevated H3K27ac at the SOX10 and NFKBIE promoters, possibly through the decreased recruitment of HDAC1 by RelA due to the elevated NFKBIE expression. As a functional validation, we tested SOX10-KO and NFKBIE-KO M397 cells, expecting that both engineered cells would develop drug tolerance to BRAFi more rapidly than the wild type counterpart. These engineered cell lines indeed behaved as expected according to our proposed mechanism (Figure 5L). Collectively, these experiments provide strong evidences for the proposed mechanism. They validate the critical role of RelA as a rapid-acting regulator of resistance development by recruiting KDM5B and HDAC1 to epigenetically suppressed SOX10 and NFKBIE expression and consequently induce BRAFi drug tolerance (Figure 5A).

\section{Baseline epigenome states correlate with the phenotypic plasticity and drug response across melanoma cell lines}

We now turn towards understanding whether the mechanism of adaptive response of M397 cells to BRAF inhibition can be generalized to other $B R A F$-mutant melanoma cell lines that exhibit varying degrees of phenotypic plasticity and baseline level of resistance to BRAFi. We first tested the generality of $\mathrm{M}_{\text {early }}$ and $\mathrm{M}_{\text {late }}$ modules in other melanoma cell by evaluating the co-occurrence of gene sets associated with two modules across the CCLE melanoma cell lines. We observed higher co-occurrence score relative to random gene permutation (Figure S6A, see STAR Method) suggesting the various gene sets associated with two modules are not specific to the cell line we studied, but similarly modulated and co-expressed across other cohorts of melanoma cells.

Furthermore, we investigated the BRAFi-induced transcriptome changes in additional six patient-derived $B R A F$-mutant cell lines, each with a unique drug-naïve phenotypic composition that varies from largely mesenchymal (M381) to in-between neural crest and melanocytic (M263), to mostly melanocytic (M229) (Su et al., 2017). In order to permit comparisons between different cells, we projected the whole transcriptome kinetic data of each cell line onto the two-dimensional space defined by $\mathrm{M}_{\text {early }}$ and $\mathrm{M}_{\text {late, }}$, similar to the two-dimensional plot in Figure 2B (Figure 6A). Cell lines at the left side displayed much higher baseline IC50 value than those at the right side, suggesting that cells at the BRAFi-induced dedifferentiated state are intrinsically resistant to BRAF inhibition. Similar trends were also observed across various $B R A F$-mutant melanoma cell lines from the GDSC database (Figure S6B), indicating cells with higher $\mathbf{M}_{\text {early }}$ and lower $\mathbf{M}_{\text {late }}$ scores are generally resistant to BRAFi. Similar to M397, upon BRAF inhibition, each cell line exhibited an initial change in the positive direction along $\mathbf{M}_{\text {early }}$, followed by a motion along the negative direction of $\mathrm{M}_{\text {late }}$ (Figure 6A), which implied some mechanistic similarities between the 
cells. However, different cell lines also exhibited widely different amplitudes of motion along this 2D landscape, reflecting large variations in transcriptome plasticity (Figure 6A). Our hypothesis was that these different amplitudes of transcriptome motion, particularly the motion along the controlling module $\mathrm{M}_{\text {early }}$, were related to the pre-treatment (baseline) epigenome state of the cells.

Mearly was enriched with early-acting epigenetic modulations that dictate the subsequent adaptive transition in M397 (Figure 2B-2E). Thus, for comparison across cell lines, motion along $\mathrm{M}_{\text {early }}$ was chosen as a surrogate of transcriptome plasticity. We correlated the BRAFi-induced motion along $\mathrm{M}_{\text {early }}$ with the baseline epigenetic characteristics of the cells. These correlations included the average chromatin accessibility (Figure 6B), the average levels of the two histone marks across all the enriched domains (Figures 6C, 6D and S6C), and the levels of two histone marks on the TSS region of SOX10 (Figures 6E, 6F and S6D). The strong correlations, particularly with $S O X 10$-specific $\mathrm{H} 3 \mathrm{~K} 4 \mathrm{me} 3 / \mathrm{H} 3 \mathrm{~K} 27$ ac signals, suggest that cellular plasticity is associated with the baseline chromatin state of the drug-naïve cells and implicate the generality of the chromatin remodeling mechanism in the adaptive resistance of melanoma cells. These findings also imply that cellular plasticity that permits adaptation to BRAFi may be encoded in cells before treatment through general and specific structural details of the chromatin.

The relationships between cellular plasticity, chromatin accessibility (Figure 6G), and adaptive resistance to BRAFi suggest that drug targeting the chromatin remodeling machinery in combination with BRAFi would arrest the adaptive transition and inhibit the development drug resistance in the most plastic cell lines (e.g. M397 or M262) but should have little effect on the least plastic lines (e.g. M381). We used a recently-developed KDM5 inhibitor CPI-455 (Vinogradova et al., 2016) and a second generation HDAC1 inhibitor Quisinostat (Arts et al., 2009) to treat the cells in combination with BRAFi vemurafenib. We employed the minimal doses of CPI-455 and Quisinostat that were sufficient to inhibit KDM5B and HDAC1 without significant cytotoxicity (Figures S6E and S6F). Clonogenic assays revealed that, in comparison with BRAFi monotherapy, both of the dual drug combinations (BRAFi + KDM5Bi or BRAFi + HDACi) could lead to a sustained growth inhibition across several epigenetically plastic melanoma cell lines, including M397 (Figure 6H). These results demonstrate the potential utility of co-targeting the driver oncogene $B R A F$ along with chromatin-remodeling machinery to treat certain melanomas which demonstrate significant epigenetic changes upon BRAF inhibition. Importantly, the M381 and M233 cell lines, which exhibited the lowest levels of plasticity (Figure 6A) and chromatin accessibility (Figure 6G), did not respond to the therapy combinations (Figures 6H). Thus, the responsiveness towards combination therapy with epigenetic drugs can be predicted through the degree of cellular plasticity, which may be encoded by the baseline epigenome of cells prior to treatment. Collectively, the strong associations across different cell lines between transcriptome changes and the baseline chromatin permissiveness suggest both a generality and predictable limitations of the molecular mechanism (Figure 5A), and also inform the use of epigenetic drugs across melanoma cells of different phenotypes.

\section{The presence of the epigenetic resistance mechanism in MAPKi-treated melanoma patients}


To determine whether the adaptive resistance mechanism identified in melanoma cell lines is present in melanoma patient samples, we collected paired tumor biopsies from patients bearing $B R A F^{V 600}$-mutant tumors prior to MAPKi treatment and at the onset of therapy resistance. Paraffinembedded cross sections were stained for MITF, SOX10, NFKBIE, and KDM5B. Prior to the therapy, these four protein markers showed relatively uniform spatial distributions (Figure 7A). After MAPKi exposure, some regions of the tumor tissues retained similar MITF, SOX10 and NFKBIE expression and loss of KDM5B, while other regions showed elevated KDM5B but loss of MITF and SOX10 (Figures 7A and 7B). The mutually exclusive spatial distribution of KDM5B and SOX10/MITF was consistent with the chromatin remodeling-mediated adaptive resistance mechanism observed (Figure 5A), and suggested the presence of the adaptive resistance mediated by the epigenetic reprogramming in melanoma patients undergoing MAPKi treatments.

To investigate the generality of our findings, we interrogated the expression levels of phenotypic markers and critical TFs using published transcriptome data of $B R A F$-mutant melanoma patients (Hugo et al., 2015; Kwong et al., 2015). Gene expression levels from seven paired samples before and after MAPKi treatment were compared and enriched against curated gene sets (Figures 7C, and S7A; Table S6; STAR Methods). The reduced expression of MITF, NFKBIE, SOX10 and other melanocytic genes as well as the elevated expression of $K D M 5 B, J U N$, and other mesenchymalrelated genes after treatment suggested the existence of the chromatin remodeling-mediated adaptive resistance in these patients. Furthermore, we also analyzed the published transcriptome data (Kwong et al., 2015) of the paired melanoma patient samples by projecting them onto the twodimensional plot defined by $\mathbf{M}_{\text {early }}$ and $\mathbf{M}_{\text {late }}$ and calculating the changes of $\mathbf{M}_{\text {early }}$ and $\mathbf{M}_{\text {late }}$ score upon MAPKi treatment. Like M397, $\mathbf{M}_{\text {early }}$ score increased and $\mathbf{M}_{\text {late }}$ score decreased after treatment, indicating the gene signatures associated with $\mathrm{M}_{\text {early }}$ and $\mathbf{M}_{\text {late }}$ displayed consistent changes with our cell line model (Figure S7B). In addition, we also evaluated the co-occurrence of gene sets associated with $\mathrm{M}_{\text {early }}$ and $\mathrm{M}_{\text {late }}$ modules across the TCGA melanoma patient samples (STAR Method). We observed higher co-occurrence score relative to random gene permutation (Figure S7C). This suggests that the various functional gene sets associated with two modules are not cell line specific, but similarly modulated and co-expressed across other cohorts of melanoma patient samples. We further performed Kaplan-Meier survival analysis using the melanoma dataset in TCGA (STAR Methods). Consistent with our mechanism, patients with either low baseline expression level of $K D M 5 B$ or higher level of $N F K B I E$ are less likely to develop adaptive resistance to MAPK inhibition and consequently have longer overall survival (Figure 7D). Taken together, these results confirmed the existence of adaptive resistance in melanoma patients, and validated the clinical relevance of the epigenetic mechanism.

\section{Discussion}

Epigenetic plasticity is precisely titrated during normal development to stabilize cell fate commitment and facilitate appropriate cellular responses to external cues (Brown et al., 2014; Flavahan et al., 2017; Li, 2002). Cancer cells with dysfunctional epigenome homeostasis can exploit this built-in chromatin plasticity to survive drug challenges and other stressful conditions 
(Dawson and Kouzarides, 2012; Easwaran et al., 2014; Lindroth et al., 2015; Wainwright and Scaffidi, 2017). Accumulating evidence indicates that epigenetic dysregulation can vary across different tumor cells and can also give rise to all the classic hallmarks of cancer (Flavahan et al., 2017). For example, for epigenetically plastic cancer cells, a highly permissive epigenetic landscape allows them to rapidly adapt to drug challenges by reversibly transitioning into a drugtolerant state that fuels malignant progression. Such adaptive transitions have been recently observed in clinical biospecimens of glioblastoma (Liau et al., 2017), breast cancer (Jordan et al., 2016), and many other tumor types (Knoechel et al., 2014; Roesch et al., 2010; Sharma et al., 2010). Despite the strong influence of epigenetic plasticity on therapy resistance, the mechanistic underpinnings of the drug-induced epigenetic reprogramming that initiates the adaptive transition are less clear. A systems-level characterization aimed at capturing the dynamic drug adaptation is a pressing need for solving this mechanistic puzzle.

Our goal was to establish a firm mechanistic link between epigenetic plasticity and the development of adaptive drug resistance in $B R A F$-mutant melanomas. Using BRAFi-treated patient-derived melanoma cell lines as models, our study revealed several properties of cellular plasticity. First, the drug-induced cell state changes were completely reversible upon drug removal at the transcriptome, epigenome, and functional levels. Second, the cell state changes proceeded via the sequential operations of two distinct gene expression programs, with the early-acting gene module setting in motion epigenetic and transcriptional programs that encode for longer-term changes associated with the late-acting gene module, ultimately yielding the drug-resistant mesenchymal-like phenotype. Finally, the activation of the early-acting module upon BRAF inhibition is extremely rapid. For M397 cells, activation involves nuclear translocation of the key transcription factor RelA, aided by rapid down-regulation of NFKBIE and coupled with the rapid recruitment of histone modifiers. These cells are thus poised with a 'hair-trigger' response to drug challenge.

A mechanistic link between epigenetic plasticity and the development of adaptive drug resistance was successfully established through a systems-level, multi-omics approach that focused on the kinetics of the adaptive response. We first acquired time-resolved transcriptome and epigenome data to track the kinetics of the reversible cell state transition over a two-month period. Critical regulators that underlie the adaptive transition were distilled from thousands of candidate TFs/co-factors through the sequential application of two distinct systems biology approaches followed by bioinformatics inferences. First, a top-down information theoretic analysis (Alter et al., 2000; Levine, 1978; Remacle et al., 2010; Zadran et al., 2013, 2014) simplified the transcriptome changes into the changes of two gene modules, one of which ( $\left.\mathrm{M}_{\text {early }}\right)$ was fully activated within the first 3 days of BRAF inhibition. Next, dynamic ODE modeling (Mutambara, 2017) precisely identified co-dependencies between two modules and uncovered the dominating influence of $\mathrm{M}_{\text {early }}$ over both itself and the second module, $\mathbf{M}_{\text {late. }}$ Guided by these gene module relationships, bioinformatic inference further identified key TFs and epigenetic regulators from which we extracted and experimentally validated a mechanistic regulatory network for the 
adaptive resistance. Such mechanistic understanding would be difficult to uncover without the synergistic integration of this three-part systems-level computational analysis. This analytic methodology could potentially be adapted for the understanding of cell state changes in other biological contexts.

We find that the adaptive response was not truly reversible, in that the trajectory that the cancer cells took upon drug exposure was not retraced following drug removal. Although drug release reversed the transcriptional changes, it did not reverse the order of the two modules, so that Mearly was deactivated first upon drug removal. Therefore, the cells took a cyclic route as they traversed from drug-naïve to drug-resistant and back (Figure 2B). Similar to the adaptive response to drugging, this finding implicates the involvement of $\mathbf{M}_{\text {early-associated epigenetic reprogramming }}$ in regulating the first step of the reverse transition as well. It emphasizes the importance of those epigenetic regulations that manifest as an early-acting transcriptional program for the rapid adaptation to therapeutic challenges in melanomas. Indeed, the sequential operation of $\mathrm{M}_{\text {early }}$ and $\mathrm{M}_{\text {late }}$ modules associated with cell state regression was generally observed across several other melanoma cell lines, although with different magnitudes of motion (Figure 6A). By interpreting that magnitude of motion as a metric of transcriptome plasticity, we found that the plasticity was strongly correlated with baseline chromatin accessibility and with the levels of activation histone marks of untreated melanoma cell lines (Figure 6B-6F). These findings imply that transcriptome plasticity may be epigenetically encoded prior to drug exposure. The sequential operations of $\mathrm{M}_{\text {early }}$ and $\mathbf{M}_{\text {late }}$ also resemble observations of sequential transcriptional waves that guide cell differentiation in other biological systems (Hnisz et al., 2017; Telley et al., 2016; Yosef et al., 2013; Zhang et al., 2019). The molecular causes of the differences in baseline epigenome profiles across cell lines are unclear. Deciphering the causes of the intertumoral epigenetic heterogeneity provides an important area for future investigation.

A distinct chromatin state was observed in the drug-induced resistant cells compared with untreated or long-term drug removal cells. We identified histone remodelers KDM5B and HDAC1 as important players for establishing the resistant epigenetic state. However, other epigenetic remodelers may also contribute to the cell state changes. In fact, the SIRT6 and BET protein families have been reported as regulators of MAPKi resistance in other melanoma systems (Fallahi-Sichani et al., 2017; Strub et al., 2018). Histone remodelers are generally recruited by TFs to regulate specific downstream genes. While other TFs could potentially recruit histone remodelers to regulate downstream resistance-associated genes, we identified RelA as a key player in regulating $S O X 10$, whose repression is well-documented to trigger the resistant mesenchymal phenotype in melanomas (Shaffer et al., 2017; Sun et al., 2014). In fact, the immediate recovery of SOX10 expression levels after inhibition of RelA nuclear translocation in drug-tolerant cells confirmed the critical role of RelA as a key upstream TF in regulating SOX10 expression and associated resistance development (Figure 5F). In addition, the rapid recruitment of histone remodelers by RelA to the promoter regions of SOX10 within as early as 3 days after BRAF inhibition confirmed the critical role of RelA for initiating the adaptive resistance early on. The 
question of how BRAF inhibition induces the subsequent RelA-dependent molecular circuit was not resolved. The elevated level of reactive oxygen species (ROS) in melanoma cells upon BRAF inhibition (Wang et al., 2018) might be relevant, since ROS is known to activate the proinflammatory NFאB signaling pathway (Gloire et al., 2006). Phosphoproteomics that can resolve early signaling events immediately after BRAF inhibition may prove useful for such endeavor (Lee et al., 2012; Wolf-Yadlin et al., 2007).

One of the most exciting aspects of epigenetic therapy is the ability to potentiate responses to existing therapies, which effectively multiplies the drug arsenal against cancer progression (Ellis et al., 2009). The intimate role of epigenetic dysregulation in therapy resistance development suggested that the epigenetic regulators KDM5B and HDAC1 would be attractive targets for combining with BRAFi for arresting the development of adaptive resistance at least in epigenetically plastic melanoma cells. This hypothesis was validated in clonogenic assays (Figure $6 \mathrm{H})$. It is worth noting that BRAF and HDAC inhibitors were reported to be used in sequential order to eliminate the melanoma cells that acquired resistance to BRAF inhibition by exploiting the lethal ROS levels (Wang et al., 2018). However, our results pointed to an alternative therapeutic strategy that using them in combination at the very beginning could retain tumor cells in the drug-sensitive stage and thus lead to sustained growth inhibition. While in vitro models may not fully recapitulate the cellular behavior in vivo, evidence of our epigenetic mechanism was also observed in melanoma tissue samples from patients under MAPK inhibitor treatments. This implies a potential role for these combination therapies in treating $B R A F$-mutant melanomas, with the provocative goal of disrupting the development of adaptive resistance against MAPKi (Figure 7A). In an interesting parallel, the adaptive regression in melanoma towards the drug-tolerant state has also been reported to contribute to resistance development in various immunotherapy regimens (Hugo et al., 2015; Landsberg et al., 2012; Mehta et al., 2018; Su et al., 2017). Indeed, by analyzing transcriptome data of melanoma patients from a recent trial of PD-1 checkpoint blockade (Hugo et al., 2016), we observed lower $\mathrm{M}_{\text {late }}$ module score in non-responders than responders (Figure S7D), implying the more dedifferentiated melanoma phenotype was less likely to respond to PD1 checkpoint blockade. With the increasing options of immunotherapy in treating metastatic melanomas in the clinic, combinations of epigenetic drugs with drugs targeting immune modulations may warrant further exploration. Moreover, how to sensitize the melanoma cells with minimal epigenetic plasticity (e.g. M381) to combinatory targeted inhibitions also requires further studies. 


\section{Acknowledgements}

We thank S. Huang (ISB) and L. Hood (ISB) for comments on the manuscript. We acknowledge the following funding agencies and foundations for support: NIH/NCI U01 CA217655 (to R.L., A.R., J.R.H., and W.W.), U54 CA199090 (to A.R., J.R.H., and W.W.), P01 CA168585 (to A.R.), R35 CA197633 (to A.R.), Andy Hill CARE Fund (to W.W.), and Phelps Family Foundation (to W.W.); Dr. Robert Vigen Memorial Fund, the Ressler Family Fund, and Ken and Donna Schultz (A.R.); the Jean Perkins Foundation (J.R.H.); ISB Innovator Award (Y.S.). National Key Research and Development Program Grant 2016YFC0900200 (to Q.S.), National Natural Science Foundation of China Grant 21775103 (to Q.S.), 81672696 (to J.G.), and 81772912 (to Y.K.). L.R. was supported by the V Foundation-Gil Nickel Family Endowed Fellowship and a scholarship from SEOM.

\section{Author Contributions}

J.R.H. and W.W. conceived the project and supervised the study. Y.S., X.L., G.L., C.L., R.N., S.W., L.R., V.L., V.L., J.C., Z.W., H.C., A.H.C.N., S.P., M.X., D.J., and J.W. performed the experiments. X.L., C.L., and C.W. performed bioinformatics analysis. Y.S., X.L., R.L., J.R.H., and W.W. established the computational models. Y.S., X.L., G.L., C.L., J.W.L., C.W., V.L., Z.W., Y.Y., G.Q., J.W., I.S., X.W., Q.S., R.L., A.R., D.B., J.G., J.R.H., and W.W. analyzed the data. Y.K., Y.X., and J.G. contributed the clinical specimens and expertise. Y.S., X.L., J.R.H., and W.W. wrote the paper. Everyone reviewed the paper.

\section{Declaration of Interests}

The authors declare no competing interests. 


\section{References}

Akbani, R., Akdemir, K.C., Aksoy, B.A., Albert, M., Ally, A., Amin, S.B., Arachchi, H., Arora, A., Auman, J.T., Ayala, B., et al. (2015). Genomic Classification of Cutaneous Melanoma. Cell.

Alter, O., Brown, P.O., and Botstein, D. (2000). Singular value decomposition for genome-wide expression data processing and modeling. Proc. Natl. Acad. Sci. 97, 10101-10106.

Anders, S., Pyl, P.T., and Huber, W. (2015). HTSeq-A Python framework to work with highthroughput sequencing data. Bioinformatics.

Arkenau, H.T., Kefford, R., and Long, G. V. (2011). Targeting BRAF for patients with melanoma. Br. J. Cancer 104, 392-398.

Arts, J., King, P., Mariën, A., Floren, W., Beliën, A., Janssen, L., Pilatte, I., Roux, B., Decrane, L., Gilissen, R., et al. (2009). JNJ-26481585, a novel "second-generation" oral histone deacetylase inhibitor, shows broad-spectrum preclinical antitumoral activity. Clin. Cancer Res. $15,6841-6851$.

Bai, X., Fisher, D.E., and Flaherty, K.T. (2019). Cell-state dynamics and therapeutic resistance in melanoma from the perspective of MITF and IFN $\gamma$ pathways. Nat. Rev. Clin. Oncol.

Barretina, J., Caponigro, G., Stransky, N., Venkatesan, K., Margolin, A.A., Kim, S., Wilson, C.J., Lehár, J., Kryukov, G. V, Sonkin, D., et al. (2012). The Cancer Cell Line Encyclopedia enables predictive modelling of anticancer drug sensitivity. Nature.

Benjamini, Y., and Hochberg, Y. (2018). Controlling the False Discovery Rate: A Practical and Powerful Approach to Multiple Testing. J. R. Stat. Soc. Ser. B.

Berger, A.J., Davis, D.W., Tellez, C., Prieto, V.G., Gershenwald, J.E., Johnson, M.M., Rimm, D.L., and Bar-Eli, M. (2005). Automated Quantitative Analysis of Activator Protein-2 $\alpha$ Subcellular Expression in Melanoma Tissue Microarrays Correlates with Survival Prediction. Cancer Res. 65, 11185-11192.

Brock, A., Chang, H., and Huang, S. (2009). Non-genetic heterogeneity a mutation-independent driving force for the somatic evolution of tumours. Nat. Rev. Genet. 10, 336-342.

Brown, R., Curry, E., Magnani, L., Wilhelm-Benartzi, C.S., and Borley, J. (2014). Poised epigenetic states and acquired drug resistance in cancer. Nat. Rev. Cancer 14, 747-753.

Buenrostro, J.D., Wu, B., Chang, H.Y., and Greenleaf, W.J. (2015). ATAC-seq: A method for assaying chromatin accessibility genome-wide. Curr. Protoc. Mol. Biol.

Cara, S., and Tannock, I.F. (2001). Retreatment of patients with the same chemotherapy: Implications for clinical mechanisms of drug resistance. Ann. Oncol. 12, 23-27.

Dawson, M.A., and Kouzarides, T. (2012). Cancer epigenetics: from mechanism to therapy. Cell $150,12-27$.

Denny, S.K., Yang, D., Chuang, C.H., Brady, J.J., Lim, J.S.S., Grüner, B.M., Chiou, S.H., Schep, A.N., Baral, J., Hamard, C., et al. (2016). Nfib Promotes Metastasis through a Widespread Increase in Chromatin Accessibility. Cell. 
Easwaran, H., Tsai, H.C., and Baylin, S.B. (2014). Cancer Epigenetics: Tumor Heterogeneity, Plasticity of Stem-like States, and Drug Resistance. Mol. Cell 54, 716-727.

Eichler, G.S., Huang, S., and Ingber, D.E. (2003). Gene Expression Dynamics Inspector (GEDI): for integrative analysis of expression profiles. Bioinformatics 19, 2321-2322.

Ellis, L., Atadja, P.W., and Johnstone, R.W. (2009). Epigenetics in cancer: Targeting chromatin modifications. Mol. Cancer Ther. 8, 1409-1420.

Fallahi-Sichani, M., Moerke, N.J., Niepel, M., Zhang, T., Gray, N.S., and Sorger, P.K. (2015). Systematic analysis of BRAFV600E melanomas reveals a role for JNK/c-Jun pathway in adaptive resistance to drug-induced apoptosis. Mol. Syst. Biol. 11, 797-797.

Fallahi-Sichani, M., Becker, V., Izar, B., Baker, G.J., Lin, J., Boswell, S.A., Shah, P., Rotem, A., Garraway, L.A., and Sorger, P.K. (2017). Adaptive resistance of melanoma cells to RAF inhibition via reversible induction of a slowly dividing de-differentiated state. Mol. Syst. Biol. 13,905 .

Fang, M., Hutchinson, L., Deng, A., and Green, M.R. (2016). Common BRAF(V600E)-directed pathway mediates widespread epigenetic silencing in colorectal cancer and melanoma. Proc. Natl. Acad. Sci. 113, 1250-1255.

Flavahan, W.A., Gaskell, E., and Bernstein, B.E. (2017). Epigenetic plasticity and the hallmarks of cancer. Science (80-. ). 357.

Forde, P.M., Chaft, J.E., Sloan, M., Cancer, K., Smith, K.N., Anagnostou, V., Cottrell, T.R., Hellmann, M.D., Sloan, M., Cancer, K., et al. (2018). Neoadjuvant PD-1 Blockade in Resectable Lung Cancer. N. Engl. J. Med.

Gloire, G., Legrand-Poels, S., and Piette, J. (2006). NF-кB activation by reactive oxygen species: Fifteen years later. Biochem. Pharmacol. 72, 1493-1505.

Hänzelmann, S., Castelo, R., and Guinney, J. (2013). GSVA: Gene set variation analysis for microarray and RNA-Seq data. BMC Bioinformatics.

Hata, A.N., Niederst, M.J., Archibald, H.L., Gomez-Caraballo, M., Siddiqui, F.M., Mulvey, H.E., Maruvka, Y.E., Ji, F., Bhang, H.E.C., Radhakrishna, V.K., et al. (2016). Tumor cells can follow distinct evolutionary paths to become resistant to epidermal growth factor receptor inhibition. Nat. Med. 22, 262-269.

Hinohara, K., Wu, H.J., Vigneau, S., McDonald, T.O., Igarashi, K.J., Yamamoto, K.N., Madsen, T., Fassl, A., Egri, S.B., Papanastasiou, M., et al. (2018). KDM5 Histone Demethylase Activity Links Cellular Transcriptomic Heterogeneity to Therapeutic Resistance. Cancer Cell 34, 939953.e9.

Hnisz, D., Shrinivas, K., Young, R.A., Chakraborty, A.K., and Sharp, P.A. (2017). A Phase Separation Model for Transcriptional Control. Cell.

Hoek, K.S., Eichhoff, O.M., Schlegel, N.C., Döbbeling, U., Kobert, N., Schaerer, L., Hemmi, S., and Dummer, R. (2008). In vivo switching of human melanoma cells between proliferative and invasive states. Cancer Res. 68, 650-656. 
Hsu, F., Kent, J.W., Clawson, H., Kuhn, R.M., Diekhans, M., and Haussler, D. (2006). The UCSC known genes. Bioinformatics.

Hugo, W., Shi, H., Sun, L., Piva, M., Song, C., Kong, X., Moriceau, G., Hong, A., Dahlman, K.B., Johnson, D.B., et al. (2015). Non-genomic and Immune Evolution of Melanoma Acquiring MAPKi Resistance. Cell 162, 1271-1285.

Hugo, W., Zaretsky, J.M., Sun, L., Song, C., Moreno, B.H., Hu-Lieskovan, S., Berent-Maoz, B., Pang, J., Chmielowski, B., Cherry, G., et al. (2016). Genomic and Transcriptomic Features of Response to Anti-PD-1 Therapy in Metastatic Melanoma. Cell 165, 35-44.

Iorio, F., Knijnenburg, T.A., Vis, D.J., Bignell, G.R., Menden, M.P., Schubert, M., Aben, N., Gonçalves, E., Barthorpe, S., Lightfoot, H., et al. (2016). A Landscape of Pharmacogenomic Interactions in Cancer. Cell.

Jordan, N.V., Bardia, A., Wittner, B.S., Benes, C., Ligorio, M., Zheng, Y., Yu, M., Sundaresan, T.K., Licausi, J.A., Desai, R., et al. (2016). HER2 expression identifies dynamic functional states within circulating breast cancer cells. Nature 537, 102-106.

Kelly, T.K., De Carvalho, D.D., and Jones, P.A. (2010). Epigenetic modifications as therapeutic targets. Nat. Biotechnol. 28, 1069-1078.

Kemper, K., De Goeje, P.L., Peeper, D.S., and Van Amerongen, R. (2014). Phenotype switching: Tumor cell plasticity as a resistance mechanism and target for therapy. Cancer Res. 74, 59375941.

Kim, D., Pertea, G., Trapnell, C., Pimentel, H., Kelley, R., and Salzberg, S.L. (2013). TopHat2: Accurate alignment of transcriptomes in the presence of insertions, deletions and gene fusions. Genome Biol. 14.

Knoechel, B., Roderick, J.E., Williamson, K.E., Zhu, J., Lohr, J.G., Cotton, M.J., Gillespie, S.M., Fernandez, D., Ku, M., Wang, H., et al. (2014). An epigenetic mechanism of resistance to targeted therapy in T cell acute lymphoblastic leukemia. Nat. Genet. 46, 364-370.

Kochanowski, K., Morinishi, L., Altschuler, S.J., and Wu, L.F. (2018). Drug persistence - From antibiotics to cancer therapies. Curr. Opin. Syst. Biol. 10, 1-8.

Kravchenko-Balasha, N., Levitzki, A., Goldstein, A., Rotter, V., Gross, A., Remacle, F., and Levine, R.D. (2012). On a fundamental structure of gene networks in living cells. Proc. Natl. Acad. Sci. 109, 4702-4707.

Kravchenko-Balasha, N., Wang, J., Remacle, F., Levine, R.D., and Heath, J.R. (2014). Glioblastoma cellular architectures are predicted through the characterization of two-cell interactions. Proc. Natl. Acad. Sci. 111, 6521-6526.

Kravchenko-Balasha, N., Shin, Y.S., Sutherland, A., Levine, R.D., and Heath, J.R. (2016a). Intercellular signaling through secreted proteins induces free-energy gradient-directed cell movement. Proc. Natl. Acad. Sci. 113, 5520-5525.

Kravchenko-Balasha, N., Johnson, H., White, F.M., Heath, J.R., and Levine, R.D. (2016b). A thermodynamic-based interpretation of protein expression heterogeneity in different glioblastoma multiforme tumors identifies tumor-specific unbalanced processes. J. Phys. Chem. 
B 120, 5990-5997.

Kurata, T., Tamura, K., Kaneda, H., Nogami, T., Uejima, H., Asai, G., Nakagawa, K., and Fukuoka, M. (2004). Effect of re-treatment with gefitinib ("Iressa", ZD1839) after acquisition of resistance [1]. Ann. Oncol. 15, 173.

Kwong, L.N., Boland, G.M., Frederick, D.T., Helms, T.L., Akid, A.T., Miller, J.P., Jiang, S., Cooper, Z.A., Song, X., Seth, S., et al. (2015). Co-clinical assessment identifies patterns of BRAF inhibitor resistance in melanoma. J. Clin. Invest. 125, 1459-1470.

Landsberg, J., Kohlmeyer, J., Renn, M., Bald, T., Rogava, M., Cron, M., Fatho, M., Lennerz, V., Wölfel, T., Hölzel, M., et al. (2012). Melanomas resist T-cell therapy through inflammationinduced reversible dedifferentiation. Nature 490, 412-416.

Langmead, B., and Salzberg, S.L. (2012). Fast gapped-read alignment with Bowtie 2. Nat. Methods.

Law, C.W., Chen, Y., Shi, W., and Smyth, G.K. (2014). Voom: Precision weights unlock linear model analysis tools for RNA-seq read counts. Genome Biol.

Lawrence, M., Huber, W., Pagès, H., Aboyoun, P., Carlson, M., Gentleman, R., Morgan, M.T., and Carey, V.J. (2013). Software for Computing and Annotating Genomic Ranges. PLoS Comput. Biol.

Lee (2012). Remarkable Effect of Gefitinib Retreatment in a Lung Cancer Patient With Lepidic Predominat Adenocarcinoma Who Had Experienced Favorable Results From Initial Treatment With Gefitinib: A Case Report. J. Clin. Med. Res.

Lee, M.J., Ye, A.S., Gardino, A.K., Heijink, A.M., Sorger, P.K., MacBeath, G., and Yaffe, M.B. (2012). Sequential application of anticancer drugs enhances cell death by rewiring apoptotic signaling networks. Cell 149, 780-794.

Levine, R.D. (1978). Information Theory Approach to Molecular Reaction Dynamics. Annu. Rev. Phys. Chem. 29, 59-92.

Li, E. (2002). Chromatin modification and epigenetic reprogramming in mammalian development. Nat. Rev. Genet. 3, 662-673.

Li, G., So, A.Y.L., Sookram, R., Wong, S., Wang, J.K., Ouyang, Y., He, P., Su, Y., Casellas, R., and Baltimore, D. (2018). Epigenetic silencing of miR-125b is required for normal B-cell development. Blood 131, 1920-1930.

Li, G., Bethune, M.T., Wong, S., Joglekar, A. V., Leonard, M.T., Wang, J.K., Kim, J.T., Cheng, D., Peng, S., Zaretsky, J.M., et al. (2019). T cell antigen discovery via trogocytosis. Nat. Methods.

Liau, B.B., Sievers, C., Donohue, L.K., Gillespie, S.M., Flavahan, W.A., Miller, T.E., Venteicher, A.S., Hebert, C.H., Carey, C.D., Rodig, S.J., et al. (2017). Adaptive Chromatin Remodeling Drives Glioblastoma Stem Cell Plasticity and Drug Tolerance. Cell Stem Cell 20, 233-246.e7.

Lim, J., and Thiery, J.P. (2012). Epithelial-mesenchymal transitions: insights from development. 
Development 139, 3471-3486.

Lindroth, A.M., Park, Y.J., and Plass, C. (2015). Epigenetic reprogramming in cancer. Epigenetics Hum. Heal. 4, 193-223.

Love, M.I., Huber, W., and Anders, S. (2014). Moderated estimation of fold change and dispersion for RNA-seq data with DESeq2. Genome Biol.

Mackiewicz-Wysocka, M., Krokowicz, Ł., Kocur, J., and Mackiewicz, J. (2014). Resistance to vemurafenib can be reversible after treatment interruption. Med. (United States) 93.

Mehta, A., Kim, Y.J., Robert, L., Tsoi, J., Comin-Anduix, B., Berent-Maoz, B., Cochran, A.J., Economou, J.S., Tumeh, P.C., Puig-Saus, C., et al. (2018). Immunotherapy resistance by inflammation-induced dedifferentiation. Cancer Discov. 8, 935-943.

Müller, J., Krijgsman, O., Tsoi, J., Robert, L., Hugo, W., Song, C., Kong, X., Possik, P.A., Cornelissen-Steijger, P.D.M., Foppen, M.H.G., et al. (2014). Low MITF/AXL ratio predicts early resistance to multiple targeted drugs in melanoma. Nat. Commun. 5.

Mutambara, A.G.O. (2017). Modeling of Dynamic Systems. In Design and Analysis of Control Systems, (CRC Press), pp. 49-148.

Nazarian, R., Shi, H., Wang, Q., Kong, X., Koya, R.C., Lee, H., Chen, Z., Lee, M.-K., Attar, N., Sazegar, H., et al. (2010). Melanomas acquire resistance to B-RAF(V600E) inhibition by RTK or N-RAS upregulation. Nature 468, 973-977.

Pisco, A.O., Brock, A., Zhou, J., Moor, A., Mojtahedi, M., Jackson, D., and Huang, S. (2013). Non-Darwinian dynamics in therapy-induced cancer drug resistance. Nat. Commun. 4.

Poovathingal, S.K., Kravchenko-Balasha, N., Shin, Y.S., Levine, R.D., and Heath, J.R. (2016). Critical Points in Tumorigenesis: A Carcinogen-Initiated Phase Transition Analyzed via SingleCell Proteomics. Small 12, 1425-1431.

Pritchard, J.R., Lauffenburger, D.A., and Hemann, M.T. (2012). Understanding resistance to combination chemotherapy. Drug Resist. Updat.

Rambow, F., Rogiers, A., Marin-Bejar, O., Aibar, S., Femel, J., Dewaele, M., Karras, P., Brown, D., Chang, Y.H., Debiec-Rychter, M., et al. (2018). Toward Minimal Residual Disease-Directed Therapy in Melanoma. Cell 174, 843-855.e19.

Ramirez, M., Rajaram, S., Steininger, R.J., Osipchuk, D., Roth, M.A., Morinishi, L.S., Evans, L., Ji, W., Hsu, C.H., Thurley, K., et al. (2016). Diverse drug-resistance mechanisms can emerge from drug-tolerant cancer persister cells. Nat. Commun. 7.

Ramírez, F., Dündar, F., Diehl, S., Grüning, B.A., and Manke, T. (2014). deepTools: a flexible platform for exploring deep-sequencing data. Nucleic Acids Res.

Ramsdale, R., Jorissen, R.N., Li, F.Z., Al-Obaidi, S., Ward, T., Sheppard, K.E., Bukczynska, P.E., Young, R.J., Boyle, S.E., Shackleton, M., et al. (2015). The transcription cofactor c-JUN mediates phenotype switching and BRAF inhibitor resistance in melanoma. Sci. Signal. 8, ra82.

Remacle, F., Kravchenko-Balasha, N., Levitzki, A., and Levine, R.D. (2010). Informationtheoretic analysis of phenotype changes in early stages of carcinogenesis. Proc. Natl. Acad. Sci. 
107, 10324-10329.

Robinson, J.T., Thorvaldsdóttir, H., Winckler, W., Guttman, M., Lander, E.S., Getz, G., and Mesirov, J.P. (2011). Integrative genomics viewer. Nat. Biotechnol.

Roesch, A., Fukunaga-Kalabis, M., Schmidt, E.C., Zabierowski, S.E., Brafford, P.A., Vultur, A., Basu, D., Gimotty, P., Vogt, T., and Herlyn, M. (2010). A Temporarily Distinct Subpopulation of Slow-Cycling Melanoma Cells Is Required for Continuous Tumor Growth. Cell 141, 583594.

Roesch, A., Vultur, A., Bogeski, I., Wang, H., Zimmermann, K.M., Speicher, D., Körbel, C., Laschke, M.W., Gimotty, P.A., Philipp, S.E., et al. (2013). Overcoming intrinsic multidrug resistance in melanoma by blocking the mitochondrial respiratory chain of slow-cycling JARID1Bhigh cells. Cancer Cell 23, 811-825.

Sáez-Ayala, M., Montenegro, M.F., Sánchez-del-Campo, L., Fernández-Pérez, M.P., Chazarra, S., Freter, R., Middleton, M., Piñero-Madrona, A., Cabezas-Herrera, J., Goding, C.R., et al. (2013). Directed Phenotype Switching as an Effective Antimelanoma Strategy. Cancer Cell 24, $105-119$.

Salgia, R., and Kulkarni, P. (2018). The Genetic/Non-genetic Duality of Drug 'Resistance' in Cancer. Trends in Cancer 4, 110-118.

Shaffer, S.M., Dunagin, M.C., Torborg, S.R., Torre, E.A., Emert, B., Krepler, C., Beqiri, M., Sproesser, K., Brafford, P.A., Xiao, M., et al. (2017). Rare cell variability and drug-induced reprogramming as a mode of cancer drug resistance. Nature 546, 431-435.

Sharma, S. V, Lee, D.Y., Li, B., Quinlan, M.P., Takahashi, F., Maheswaran, S., McDermott, U., Azizian, N., Zou, L., Fischbach, M.A., et al. (2010). A chromatin-mediated reversible drugtolerant state in cancer cell subpopulations. Cell 141, 69-80.

Shin, H.M., Kim, M.H., Kim, B.H., Jung, S.H., Kim, Y.S., Park, H.J., Hong, J.T., Min, K.R., and Kim, Y. (2004). Inhibitory action of novel aromatic diamine compound on lipopolysaccharide-

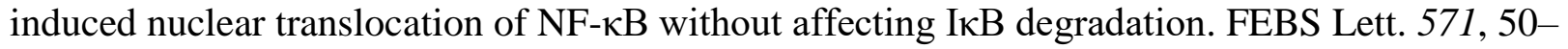
54.

Spiecker, M., Darius, H., and Liao, J.K. (2000). A Functional Role of I $\kappa$ B- $\varepsilon$ in Endothelial Cell Activation. J. Immunol. 164, 3316-3322.

Strub, T., Ghiraldini, F.G., Carcamo, S., Li, M., Wroblewska, A., Singh, R., Goldberg, M.S., Hasson, D., Wang, Z., Gallagher, S.J., et al. (2018). SIRT6 haploinsufficiency induces BRAF V600E melanoma cell resistance to MAPK inhibitors via IGF signalling. Nat. Commun. 9.

Su, Y., Wei, W., Robert, L., Xue, M., Tsoi, J., Garcia-Diaz, A., Homet Moreno, B., Kim, J., Ng, R.H., Lee, J.W., et al. (2017). Single-cell analysis resolves the cell state transition and signaling dynamics associated with melanoma drug-induced resistance. Proc. Natl. Acad. Sci. 114, 1367913684.

Subramanian, A., Tamayo, P., Mootha, V.K., Mukherjee, S., Ebert, B.L., Gillette, M.A., Paulovich, A., Pomeroy, S.L., Golub, T.R., Lander, E.S., et al. (2005). Gene set enrichment analysis: A knowledge-based approach for interpreting genome-wide expression profiles. Proc. Natl. Acad. Sci. 102, 15545-15550. 
Sun, C., Wang, L., Huang, S., Heynen, G.J.J.E., Prahallad, A., Robert, C., Haanen, J., Blank, C., Wesseling, J., Willems, S.M., et al. (2014). Reversible and adaptive resistance to BRAF(V600E) inhibition in melanoma. Nature 508, 118-122.

Suvà, M.L., Rheinbay, E., Gillespie, S.M., Patel, A.P., Wakimoto, H., Rabkin, S.D., Riggi, N., Chi, A.S., Cahill, D.P., Nahed, B. V., et al. (2014). Reconstructing and reprogramming the tumor-propagating potential of glioblastoma stem-like cells. Cell 157, 580-594.

Telley, L., Govindan, S., Prados, J., Stevant, I., Nef, S., Dermitzakis, E., Dayer, A., and Jabaudon, D. (2016). Sequential transcriptional waves direct the differentiation of newborn neurons in the mouse neocortex. Science (80-. ). 351, 1443-1446.

Das Thakur, M., Salangsang, F., Landman, A.S., Sellers, W.R., Pryer, N.K., Levesque, M.P., Dummer, R., McMahon, M., and Stuart, D.D. (2013). Modelling vemurafenib resistance in melanoma reveals a strategy to forestall drug resistance. Nature 494, 251-255.

Tsoi, J., Robert, L., Paraiso, K., Galvan, C., Sheu, K.M., Lay, J., Wong, D.J.L., Atefi, M., Shirazi, R., Wang, X., et al. (2018). Multi-stage Differentiation Defines Melanoma Subtypes with Differential Vulnerability to Drug-Induced Iron-Dependent Oxidative Stress. Cancer Cell 33, 890-904.e5.

Uribe, R.A., and Bronner, M.E. (2015). Meis3 is required for neural crest invasion of the gut during zebrafish enteric nervous system development. Mol. Biol. Cell 26, 3728-3740.

Vasudevan, S., Flashner-Abramson, E., Remacle, F., Levine, R.D., and Kravchenko-Balasha, N. (2018). Personalized disease signatures through information-theoretic compaction of big cancer data. Proc. Natl. Acad. Sci. 115, 7694-7699.

Verfaillie, A., Imrichova, H., Atak, Z.K., Dewaele, M., Rambow, F., Hulselmans, G., Christiaens, V., Svetlichnyy, D., Luciani, F., Van den Mooter, L., et al. (2015). Decoding the regulatory landscape of melanoma reveals TEADS as regulators of the invasive cell state. Nat. Commun. 6, 6683.

Vinogradova, M., Gehling, V.S., Gustafson, A., Arora, S., Tindell, C.A., Wilson, C., Williamson, K.E., Guler, G.D., Gangurde, P., Manieri, W., et al. (2016). An inhibitor of KDM5 demethylases reduces survival of drug-tolerant cancer cells. Nat. Chem. Biol. 12, 531-538.

Wagle, N., Emery, C., Berger, M.F., Davis, M.J., Sawyer, A., Pochanard, P., Kehoe, S.M., Johannessen, C.M., MacConaill, L.E., Hahn, W.C., et al. (2011). Dissecting therapeutic resistance to RAF inhibition in melanoma by tumor genomic profiling. J. Clin. Oncol. 29, 30853096.

Wainwright, E.N., and Scaffidi, P. (2017). Epigenetics and Cancer Stem Cells: Unleashing, Hijacking, and Restricting Cellular Plasticity. Trends in Cancer 3, 372-386.

Wang, L., Leite de Oliveira, R., Huijberts, S., Bosdriesz, E., Pencheva, N., Brunen, D., Bosma, A., Song, J.Y., Zevenhoven, J., Los-de Vries, G.T., et al. (2018). An Acquired Vulnerability of Drug-Resistant Melanoma with Therapeutic Potential. Cell 173, 1413-1425.e14.

Warden, C.D., Yuan, Y.-C., and Wu, X. (2013). Optimal Calculation of RNA-Seq Fold-Change Values. Int. J. Comput. Bioinforma. Silico Model. 
Whiteside, S.T. (1997). I kappa B epsilon, a novel member of the Ikappa B family, controls RelA and cRel NF-kappa B activity. EMBO J. 16, 1413-1426.

Wilkerson, M.D., and Hayes, D.N. (2010). ConsensusClusterPlus: A class discovery tool with confidence assessments and item tracking. Bioinformatics.

Wolf-Yadlin, A., Hautaniemi, S., Lauffenburger, D.A., and White, F.M. (2007). Multiple reaction monitoring for robust quantitative proteomic analysis of cellular signaling networks. Proc. Natl. Acad. Sci. 104, 5860-5865.

Yosef, N., Shalek, A.K., Gaublomme, J.T., Jin, H., Lee, Y., Awasthi, A., Wu, C., Karwacz, K., Xiao, S., Jorgolli, M., et al. (2013). Dynamic regulatory network controlling T H 17 cell differentiation. Nature 496, 461-468.

Zadran, S., Remacle, F., and Levine, R.D. (2013). miRNA and mRNA cancer signatures determined by analysis of expression levels in large cohorts of patients. Proc. Natl. Acad. Sci. 110, 19160-19165.

Zadran, S., Arumugam, R., Herschman, H., Phelps, M.E., and Levine, R.D. (2014). Surprisal analysis characterizes the free energy time course of cancer cells undergoing epithelial-tomesenchymal transition. Proc. Natl. Acad. Sci. 111, 13235-13240.

Zhang, K., Wang, M., Zhao, Y., and Wang, W. (2019). Taiji: System-level identification of key transcription factors reveals transcriptional waves in mouse embryonic development. Sci. Adv. 5, eaav3262.

Zhang, Y., Liu, T., Meyer, C.A., Eeckhoute, J., Johnson, D.S., Bernstein, B.E., Nussbaum, C., Myers, R.M., Brown, M., Li, W., et al. (2008). Model-based analysis of ChIP-Seq (MACS). Genome Biol. 


\section{EXPERIMENTAL MODEL AND SUBJECT DETAILS}

\section{Cell culturing}

M-series patient-derived cell lines used in this study were generated under UCLA institutional review board approval \# 11-003254. Cells were cultured in a water-saturated incubator at $37^{\circ} \mathrm{C}$ with $5 \% \mathrm{CO}_{2}$ in RPMI 1640 with L-glutamine (Life Technologies), supplemented with 10\% fetal bovine serum (Omega), and 0.2\% antibiotics (MycoZapTM Plus-CL from Lonza). The cell lines were tested for mycoplasma and were periodically authenticated to its early passage using GenePrint 10 System (Promega). BRAF inhibitor (vemurafenib), KDM5B inhibitor (CPI-455), HDAC inhibitor (Quisinostat) and RelA translocation inhibitor (JSH-23), all from Selleck Chemicals LLC, were dissolved in DMSO at designated concentrations before applying to cell culture media. Cells were plated in $10 \mathrm{~cm}$ tissue culture plate at $60 \%$ confluency and treated with certain drugs for the specified numbers of days. M397 cells were treated with $3 \mu \mathrm{M}$ of vemurafenib for 59 days or for 29 days followed by drug removal removed and cell culture with normal medium for another 35 days. Gender of the patients from whom the cell lines were derived: M397, female; M229, male; M262, female; M249, female; M263, female; M233, male; M381, male.

\section{Patient samples}

Melanoma samples before treatment were obtained from surplus biopsies stored in the melanoma biobank at the Peking University Cancer Hospital and Institute (Beijing, China). The patient \#1 received vemurafenib and patient \#2 received dabrafenib and trametinib combinations. Both patients exhibited partial response (PR) to these MAPK inhibitors. The secondary biopsies were performed when patients showed progressive disease (PD). The patients consented to the use of their biopsy materials for scientific studies and all research was conducted in accordance to the guidelines and protocols approved by the institutional ethics review committee and abiding by all local laws for research on human derived tissue. Gender of the reported patient samples: patient\#1, female; patient\#2, female.

\section{METHOD DETAILS}

\section{RNA-seq}

Total RNA was extracted from cell pellets using RNeasy Mini Kit (Qiagen). RNA sequencing libraries were prepared with Kapa RNA mRNA HyperPrep kit (Kapa Biosystems) according to the manufacturer's protocol. Briefly, $100 \mathrm{ng}$ of total RNA from each sample was used for polyA 
RNA enrichment using magnetic oligo-dT beads. The enriched mRNA underwent fragmentation using heat and magnesium, and the first-strand cDNA was made using random priming. The combined 2nd cDNA synthesis with dUTP and A-tailing reaction generated the resulting ds cDNA with dAMP to the 3' ends. The barcoded adaptors (Illumina) finally were ligated to the ds cDNA fragments. A 10-cycle of PCR was performed to produce the final sequencing library. The libraries were validated with the Agilent Bioanalyzer DNA High Sensitivity Kit and quantified with Qubit.

\section{ATAC-seq}

A previously published protocol (Buenrostro et al., 2015) was used for cell lysis, tagmentation, and DNA purification. The Tn5 treated DNA was amplified with a 5-cycle PCR in 50 4 l reaction volumes. The tubes were removed from thermocycler and used $5 \mu \mathrm{l}$ of a partially amplified library to perform qPCR to determine how many additional PCR cycles were needed. For the samples in this study, an additional 4-5 cycles of PCR was performed on the remaining 45ul of each partially amplified product. 1.8X AmpurXP beads purification was used for the final PCR cleanup. The libraries were validated with the Agilent Bioanalyzer DNA High Sensitivity Kit, and quantified with qPCR.

\section{ChIP-seq, ChIP-PCR, and ChIP-qPCR}

H3K4me3, H3K27ac, NFkB p65, KDM5B, and HDAC1 ChIP were performed by using Magna ChIP A/G Chromatin Immunoprecipitation Kit. Briefly, cells were cultured to $\sim 80 \%$ confluency in a petri dish containing $10 \mathrm{~mL}$ of growth media and then fixed in $1 \%$ formaldehyde by adding $275 \mu \mathrm{l}$ of $37 \%$ formaldehyde for 10 minutes to cross-link protein-DNA complexes at room temperature. The unreacted formaldehyde was quenched by adding glycine to a final concentration 0.125 M. Gently swirl dish to mix. The nuclear pellet was isolated with Cell Lysis Buffer. The pellet was resuspended with $500 \mu \mathrm{l}$ SDS Lysis Buffer containing 1X Protease Inhibitor Cocktail II before sonication for 4 min (10 s on, $30 \mathrm{~s}$ off, $10 \%$ strength in a Bioruptor to yield DNA fragments of $0.2-1.0 \mathrm{~kb}$ in length. The lysates were cleared by centrifugation $\left(12,000 \mathrm{~g}\right.$ for $10 \mathrm{~min}$ at $\left.4{ }^{\circ} \mathrm{C}\right)$ and diluted tenfold in ChIP dilution buffer to decrease the concentration of SDS. After keeping $10 \%$ of the sample as input, $500 \mu \mathrm{l}$ supernatant was incubated overnight at $4{ }^{\circ} \mathrm{C}$ with antibody and $20 \mu \mathrm{L}$ of fully resuspended protein $\mathrm{A} / \mathrm{G}$ magnetic beads. The washing, elution, reverse crosslinking, and purification steps were performed according to the manufacturer's description. Eluted DNA was quantified by Qubit dsDNA HS Assay Kit, and used for further PCR, qPCR or ChIPseq library preparation.

ChIP-seq libraries were prepared with Kapa DNA HyperPrep Kit (Kapa, Cat KK 8700) according to the manufacturer's protocol. Briefly, 5-10 ng of immunoprecipitated DNA was underwent endrepaired, A tailing and adaptor ligation. A 10 cycles of PCR was performed to produce the final 
sequencing library. The libraries were validated with the Agilent Bioanalyzer DNA High Sensitivity Kit and quantified with Qubit.

ChIP-PCR was performed by using KAPA Taq ReadyMix PCR Kit on CFX96 Real Time PCR Detection System without adding plate read. ChIP qPCR was performed by using SsoAdvanced Universal SYBR Green Supermix on CFX96 Real-Time PCR Detection System. In each $\mathrm{PCR} / \mathrm{qPCR}$ reaction, $2 \mu$ eluted DNA was added.

\section{Sequencing of RNA-seq, ChIP-seq, and ATAC-seq library}

RNA and ChIP-seq library templates were prepared for sequencing using Illumina HiSeq SR Cluster V4 Kit. Sequencing runs were performed on Illumina Hiseq 2500 in the single read mode of 51cycle of read1 and 7 cycles of index read with SBS V4 Kits. ATAC-seq library templates were prepared for sequencing with Illumina HiSeq PE Cluster V4 Kit, sequencing runs were performed in the paired-end mode of 101cycle on Illumina HiSeq 2500 with HiSeq SBS V4 Kits. Real-time analysis (RTA) 2.2.38 software was used to process the image analysis and base calling.

\section{CellTiter-Glo}

5000k cells were seeded onto each well of a 96well plate and were treated with indicated drug concentrations for 72hours. ATP-based CellTiter-Glo (Promega) luminescent cell viability assay was utilized to quantify the cell number for constructing dose-response curves. IC50 values were calculated as standard from at least three biological replicates.

\section{Cell cycle and apoptosis assays}

For cell cycle analysis, 500k cells were plated and were then treated with EdU. After treatment, cells were washed with PBS and fixed. Next, cells were processed for EdU detection using the Click-iT EdU Alexa Fluor 488 Flow Cytometry Assay Kit (Thermo Fisher) according to the manufacturer's protocol. DNA content was visualized using SYTOX AADvanced (Thermo Fisher). Gates were determined using an unstained control. All experiments were performed with at least two biological replicates.

Cell apoptosis assays were performed by treating indicated cell lines cultured under respective conditions. Cells were stained with Annexin V-FITC and propidium iodide for 15 minutes at room temperature before flow cytometry analysis. Gates were determined using an unstained control. All experiments were performed with at least two biological replicates. 


\section{Fluorescence imaging of cell lines}

Fluorescent micrographs of cells were acquired with a Nikon C2plus confocal microscope (Ti) using Plan Apo $\lambda 20 \times$ objective (Nikon Inc., Melville, NY) controlled by NIS elements AR software (4.51.00) with the following settings: $30 \mu \mathrm{m}$ pin hole, 12-bit acquisition, 25-30 PMT gain, and laser power of $0.7 \%(405 \mathrm{~nm}), 1.0 \%(488 \mathrm{~nm})$, or $0.4 \%(640 \mathrm{~nm})$. The cells adhered on gelatin-coated glass surfaces in 96-well glass bottom plates (Greiner Sensoplate Plus, Cat\# 655892). To prepare the surface, $100 \mu \mathrm{L}$ of $0.1 \%$ gelatin solution was incubated in each well at room temperature for 10 minutes. After incubation, the solution was removed, and the wells were air-dried for at least 15 minutes. Typically, 10,000 cells were seeded per well in $100 \mu \mathrm{L}$ culture media and grown to $\sim 70 \%$ confluency. To fix the cells, equal volumes of $4 \%$ PFA solution was gently added to each well. After fixing for 20 minutes at room temperature, the cells were washed twice in wash buffer (0.1\% BSA in PBS), and blocked and permeabilized in blocking buffer (10\% normal donkey serum, $0.3 \%$ Triton X-100 in PBS) for 45 minutes at room temperature. After removing blocking buffer, cells were incubated in mouse anti-MITF primary antibodies (Thermo Fisher Scientific, Cat\# MA5-14154) diluted to $5 \mu \mathrm{g} / \mathrm{mL}$ in antibody diluent (1\% BSA, 1\% normal donkey serum, $0.3 \%$ Triton X-100 in PBS) for 4 hours at room temperature. After washing twice in wash buffer, cells were incubated in donkey anti-Mouse $\mathrm{IgG}$, Alexa Fluor 647 secondary antibody (Thermo Fisher Scientific, Cat\# A31571, RRID:AB_162542) diluted to $4 \mu \mathrm{g} / \mathrm{mL}$ in antibody diluent for 1 hour at room temperature. After washing twice in washer buffer, cells were counterstained for $20 \mathrm{~min}$ at room temperature with Alexa Fluo 488 Phalloidin (Thermo Fisher Scientific Cat\# A12379), as per manufacturer's instructions. After washing twice in wash buffer, cells were further counterstained for $5 \mathrm{~min}$ with 4',6-Diamidino-2-Phenylindole (DAPI) (Thermo Fisher Scientific Cat\# D1306) diluted to $1 \mu \mathrm{g} / \mathrm{mL}$ in PBS. Finally, after washing twice in PBS, the wells were filled with $78 \%$ glycerol.

\section{Western blotting}

Histone proteins were extracted using the Histone Extraction Kit (ab113476). The Invitrogen precast gel system NuPAGE was used for SDS-PAGE. The 4-12\% Bis-Tris gels were loaded with samples. After blotting, the membranes were blocked in 5\% BSA with TBS $+0.1 \%$ Tween-20 (TBST) mix for at least 1 hour at room temperature. Membranes were then incubated overnight with the primary antibody in $5 \%$ BSA with TBST at $4{ }^{\circ} \mathrm{C}$. The next day, membranes were washed three times for $5 \mathrm{~min}$ in TBST, incubated with a suitable HRP-coupled secondary antibody for 1 hour at room temperature, washed three times and proteins were visualized with SuperSignal ${ }^{\mathrm{TM}}$ West Pico PLUS Chemiluminescent Substrate (Cat.No.34577) using the ChemiDoc ${ }^{\text {TM }}$ XRS+ System. 


\section{RT-qPCR}

For quantitative reverse transcription-polymerase chain reaction (qRT-PCR), total RNA was extracted by TRIzol ${ }^{\mathrm{TM}}$ Plus RNA Purification Kit (Cat.No.12183555) and reversed to cDNAs. Real-time PCR was performed with gene-specific primers on the two-color real-time PCR detection system (BIO-RAD) using the SsoAdvanced ${ }^{\mathrm{TM}}$ Universal SYBR ${ }^{\circledR}$ Green Supermix (Cat.No.1725272) to represent the relative expression levels.

\section{Co-IP and protein detection}

For cell lysis, cells were cultured to $\sim 80 \%$ confluency in a petri dish containing $10 \mathrm{~mL}$ of growth media and were washed with ice-cold PBS three times. Then the cells were collected with a scraper in $1 \mathrm{~mL}$ ice-cold PBS supplemented with $1 \mathrm{X}$ proteinase inhibitor cocktail (Cell Signalling) and centrifuged. The cell pellets were resuspended in cell lysis buffer containing $50 \mathrm{mM}$ Tris- $\mathrm{HCl} \mathrm{pH}$ 7.5, $250 \mathrm{mM} \mathrm{NaCl}, 1 \mathrm{mM}$ EDTA, $0.5 \%$ Triton X-100, 10\% glycerol, and $1 \mathrm{X}$ proteinase inhibitor cocktail (Cell Signalling). The resuspended cell pellets were incubated in a cold room $\left(4^{\circ} \mathrm{C}\right)$ for $30 \mathrm{~min}$ and sonicated in ice-water bath three times for 5-second pulses each. Then the cell lysates were cleared by $10,000 \times \mathrm{g}$ centrifuge at $4^{\circ} \mathrm{C}$ for $10 \mathrm{~min}$. The protein was quantified by Qubit Protein Assay Kit (Invitrogen).

For cross-linking antibody to magnetic beads, $20 \mu \mathrm{l}$ magnetic protein A/G beads (Millipore) were washed with cell lysis buffer twice and resuspended in $100 \mu$ cell lysis buffer without glycerol. 5 $\mu \mathrm{g}$ Anti-NFkB p65 (RelA) antibody was coupled to Magnetic protein A/G beads by incubation at $4^{\circ} \mathrm{C}$ overnight on a rotator. The RelA antibody-coupled Protein A/G beads were washed three times in $200 \mu \mathrm{L}$ Conjugation Buffer (20 mM Sodium Phosphate, 0.15M NaCl, pH 7.5). Then the RelA antibody-coupled beads were suspended in $250 \mu \mathrm{L} 5 \mathrm{mM}$ BS3 with conjugation buffer and incubated at room temperature for $30 \mathrm{~min}$ with rotation. The cross-linking reaction was quenched by adding $12.5 \mu \mathrm{l} 1 \mathrm{M}$ Tris- $\mathrm{HCl}(\mathrm{pH} \mathrm{7.5)}$ and incubated at room temperature for $15 \mathrm{~min}$ with rotation. The RelA antibody conjugated protein A/G beads were washed with Cell Lysis buffer three times.

For co-immunoprecipitation (Co-IP) experiment, $200 \mu \mathrm{l}$ pre-cleared cell lysates were added to RelA antibody conjugated protein $\mathrm{A} / \mathrm{G}$ beads and incubated overnight at $4{ }^{\circ} \mathrm{C}$ with rotation. The beads were then washed 5 times with $500 \mu \mathrm{l}$ cell lysis buffer without glycerol. The pellet beads were collected by a magnetic stand and resuspended in $65 \mu \mathrm{l}$ SDS buffer (50mM Tris-HCl pH6.8, $2 \%$ SDS, $10 \%$ glycerol, $1 \% \beta$-mercaptoethanol).

For immunoblotting, the elutes were boiled for $10 \mathrm{~min}$ at $95^{\circ} \mathrm{C}$. The $20 \mu \mathrm{l}$ boiled elutes were electrophoresed on 10\% Mini-PROTEAN TGX Precast Gels with running buffer containing SDS. 
Then the gels were transferred on the PVDF membranes in Bio-Rad Wet Blotting Systems. The membranes were blocked with 5\% non-fat dried milk (Bio-Rad) dissolved in PBS for $1 \mathrm{~h}$ at room temperature and incubated at $4{ }^{\circ} \mathrm{C}$ overnight with the following primary antibodies: JARID1B/KDM5B (Bethyl Lab \#A301-813A), NFkB p65 (Millipore \# 17-10060), HDAC1(Millipore \# 17-608). After incubating with secondary Goat anti-Mouse/Rabbit antibodies coupled with HRP (Thermo), membranes were visualized by ChemiDoc XRS+ Imaging Systems.

\section{CRISPR engineering of cell lines}

LentiCRISPR v2 plasmids targeting the coding sequence of $K D M 5 B$ or NFKBIE, and control LentiCRISPR v2 plasmid were purchased from GenScript. Lentiviruses were produced in HEK293T cells by transient transfection of LentiCRISPR v2 plasmid and their packaging vectors psPAX2 and pMD2.G as previously described ( $\mathrm{Li}$ et al., 2019). The virus was collected, filtered through a $0.45 \mu \mathrm{m}$ syringe filter after 48 hours and the M397 cells were spin-infected with viral supernatant supplemented with $10 \mu \mathrm{g} / \mathrm{mL}$ polybrene at $2,500 \mathrm{rpm}$ and $30^{\circ} \mathrm{C}$ for $90 \mathrm{~min}$. The transduced cells were selected using puromycin, starting at 3 days post-transduction. Genome editing in the respective locus was examined using a surveyor assay, which was performed according to the manufacturer's instructions (Integrated DNA Technologies) (Li et al., 2018).

\section{Clonogenic assay}

Melanoma cells were plated onto six-well plates with fresh media at an optimal confluence. The media (with drug or DMSO) were replenished every two days. Upon the time of staining, $4 \%$ paraformaldehyde was applied onto colonies to fix the cells and $0.05 \%$ crystal violet solution was used for staining the colonies.

\section{Patient multiplexed IHC and quantification}

Multiplexed IHC staining was performed on FFPE tissue samples from melanoma primary tumors and metastatic lesions. Multiplexed IHC staining and antibody validation were performed by PerkinElmer. Briefly, the slides were firstly deparaffinized in xylene, followed by treatment with microwave for epitope recovery. Hematoxylin and eosin (H\&E) staining was performed for histopathological evaluation and multiplexed IHC staining was then conducted on the slides via an Opal 7-Color IHC Kit (NEL811001KT, PerkinElmer) and a panel of antibodies including antiKDM5B (Sigma-Aldrich), anti-MITF (Sigma-Aldrich), anti-SOX10 (Sigma-Aldrich) and antiNFKBIE (Sigma-Alrich). The protocol was based on the manual of PerkinElmer Opal staining Kit and previous studies (Forde et al., 2018). Finally, DAPI (PerkinElmer) was stained to visualize cell nuclei. Images were acquired using a Vectra Polaris Multispectral Imaging System (PerkinElmer) for whole-slide scanning. inForm Image Analysis software (inForm 2.4, 
PerkinElmer) was used to process and analyze all images. Image $\mathrm{J}$ was employed to quantitate the fluorescence intensities of cells in the designated areas.

\section{QUANTIFICATION AND STATISTICAL ANALYSIS}

\section{RNA-seq analysis}

Reads were aligned against the human genome (hg19) using TopHat2 (Kim et al., 2013). Read counts were quantified using htseq-count (Anders et al., 2015), with known gene annotations from UCSC (Hsu et al., 2006) with anti-sense (AS) genes removed. Fold-change values were calculated from Fragments Per Kilobase per Million reads(FPKM) (Warden et al., 2013) normalized expression values, which were also used for visualization (following a $\log 2$ transformation). Aligned reads were counted using GenomicRanges (Lawrence et al., 2013). Separate comparison p-values were calculated from raw counts using limma-voom (Law et al., 2014), and false discovery rate (FDR) values were calculated using the method of Benjamini and Hochberg (Benjamini and Hochberg, 2018). Prior to p-value calculation, genes were filtered to only include transcripts with an FPKM expression level of 0.1 (after a rounded log2-transformation) in at least $50 \%$ of samples (Warden et al., 2013). Genes were defined as differentially expressed if they had a $\mid$ fold-change $\mid>1.5$ and FDR $<0.05$. Candidate genes were selected based upon the inverse overlap between the early and late time series. The "Early Drug" time series included 5 samples from Day 3 to Day 29 (with active drug treatment). The "Late No-Drug" had 6 time-points after drug removal at Day 29 (4-35 days post-drug removal). There were also 3 samples with active drug treatment after Day 29, but no genes were differentially expressed for that comparison consistent with the expectation of similar gene expression patterns after developing resistance; however, those 3 late drug samples (along with an untreated control sample) were used for visualization in a heatmap of candidate genes (defined as genes with an significant increase in expression with drug treatment and a significant decrease in expression after drug removal, or a significant decrease in expression with drug treatment and a significant increase in expression after drug removal).

A heatmap of $\log _{2}(\mathrm{FPKM}+0.1)$ standardized expression (mean of 0 , standard deviation of 1 , pergene) was visualized using the 'ggplots' package in R. More specifically, standardized expression was limited to be within the range of -2 and 2 (so, all values less than -2 were set to -2 , and all values greater than 2 were set to 2), and clustering was only performed by genes (with samples ordered by time, within each category). Hierarchical clustering was performed using Euclidian Distance as the distance metric. 


\section{Consensus clustering of M397 transcriptome}

To inspect the similarity of the transcriptome of M397 in different time points, we applied consensus clustering using the R package of ConsensusClusterPlus (Wilkerson and Hayes, 2010) to define clusters. The top 3,000 most varying genes were used for consensus clustering with the hierarchical clustering method.

\section{Analysis of differentially expressed transcription factors}

To annotate differentially expressed transcription factors/co-factors, the differential expression analysis was performed by Cuffdiff among D0, DR30, DR35, D29, and D33 (FDR $\leq$ 0.05). Then we downloaded TFs/co-factors list from AnimalTFDB 3.0, and got the up/down-regulated TFs/cofactors by screened from the differential expression analysis. The result was visualized by a volcano plot.

\section{ChIP-seq analysis}

Reads were mapped to the human genome hg19 by bowtie2 (Langmead and Salzberg, 2012). The identical aligned reads were deduplicated to avoid PCR duplicates. Peaks were called on the merged set of all ChIP-seq reads of M397 using MACS2 with the following parameters: -nomodel, --broad (Zhang et al., 2008). Peaks were assigned to the gene with closest TSS. Differential analysis between D0 and any other samples( D3, D32, DR) were performed using diffReps with a window size 1000.(Love et al., 2014). Differential binding regions were called if the absolute log value of the fold change was more than 1 and FDR $<0.05$. Then the differential binding regions were compared and merged to ChIP peaks called form MACS2 To visualize peaks in each sample, bed graph file was generated using MACS2 with following parametes: --nomodel, --broad, --bdg, --SPMR. Then the generated bed graph file was converted into bigwig file by bedGraphToBigWig tool. The average of ChIP-seq signal was calculated and visualized by deepTools v3.0.2 (Ramírez et al., 2014). The read counts were normalized by RPKM. RPKM (per bin) $=$ number of reads per bin / (number of mapped reads (in millions) * bin length (kb)). To assess how $\mathrm{H} 3 \mathrm{~K} 4 \mathrm{me} 3$ and $\mathrm{H} 3 \mathrm{~K} 27 \mathrm{ac}$ signal of differential binding regions in the cyclic transition of M397 changed in multiple cell lines, we calculated the normalized read counts around the differential binding peaks $(+/-1 \mathrm{~kb})$ at the window size of $10 \mathrm{bp}$. The different peaks are called from the time-series ChIP-seq data we generated at this paper. To evaluate the average H3K4me 3 and $\mathrm{H} 3 \mathrm{~K} 27 \mathrm{ac}$ signal at $S O X 10$, we slid the $10 \mathrm{bp}$ window size from $-3 \mathrm{~Kb}$ to $+10 \mathrm{~Kb}$ around the TSS to counts the normalized reads and calculated the average value for 520-780 bins. 


\section{ATAC-seq analysis}

All experiments were performed once. First, adaptor sequences were trimmed form the reads using Cutadapt. Then Reads were aligned to hg19 with bowtie2 with standard parameters and a maximum fragment length of 2,000. (Langmead and Salzberg, 2012). The identical aligned reads were deduplicated to avoid PCR duplicates. These de-duplicated reads were then filtered for high quality (MAPQ $\geq 30$ ). Peaks were called on the merged set of all ATAC-seq reads of M397 using MACS2 with following parameters: --nomodel, -broad, -q 1e-5 (Zhang et al., 2008) and filtered to remove putative copy number varied regions (Denny et al., 2016). Differentially accessible regions between D0 and any other samples( D3, D32, DR) were identified using diffReps with a window size 500. (Shen et al., 2013)). Differential binding regions were called if the absolute log value of the fold change was more than 1 and FDR $<0.05$. Then the differential binding regions were compared and merged to ChIP peaks called form MACS2. To visualize peaks in each sample, the same routine in ChIP-seq analysis was applied. ATAC-seq profile of differentially accessible region in samples of M397 were generated by using ngs.plot.r with following parameters: -G hg19 -R bed -L 1000 -GO km -KNC 4 -SC 0,3.5.The profile of unchanged ATAC-seq peaks in samples of M397 was plotted by using ngs.plot.r with following parameters; -G hg19 -R bed -L 1000 -GO total -SC 0,3.5. HOMER was used to find over-represented motifs in the set of differentially accessible peaks by using a background set of peaks that did not significantly change, and using the parameter "-size given -len 6,8,10,12 -mset vertebrates -bg" (Denny et al., 2016). The average of ATAC-seq signal was calculated and visualized by deepTools v3.0.2 (Ramírez et al., 2014). The read counts were normalized by RPKM. RPKM (per bin)=number of reads per bin / (number of mapped reads (in millions) * bin length $(\mathrm{kb})$ ). For the calculation of the average ATAC-seq signal, we constructed the meaningful value around the different peaks $(+/-1 \mathrm{~Kb})$ at the window size of 10bp and calculated the average value for 140-160 bins. The different peaks are called from the time-series ATAC-seq data we generated at this paper.

\section{Inference of RelA downstream transcription factors}

To identify RelA-binding TFs/co-factors in the $\mathrm{M}_{\text {late }}$ process, we downloaded TF/co-factor list from AnimalTFDB 3.0. HOMER was used to annotate RelA-binding motif (HOMER Motif 208) at the whole genome level with the following parameters: annotatePeaks.pl tss hg19 -size 1800,400. Then, TF/co-factors containing RelA-binding-motif were selected out. Within this list of TF/co-factors, RelA-motif overlapped H3K4me3, H3K27ac and ATAC-seq peaks were analyzed by bedtools v2.27.1. Thirty-six TFs showed changes in the RelA-motif overlapped peaks across the adaptive transition (D32 vs D0). However, only two TFs, SOX10 and DNAJC1, displayed significant changes $(p<0.05)$ for all three epigenome alterations (Table S5). 


\section{Surprisal analysis and SOM visualization}

In order to analyze the dynamic transcriptome changes across all time points, we assume that many of them are coordinately changing together as a group (or gene module). Surprisal analysis has been well documented in deconvoluting the change of thousands of genes into the change of a couple of gene modules and one unchanged gene expression baseline (Remacle et al., 2010; Vasudevan et al., 2018; Zadran et al., 2013, 2014).

When applied here, surprisal analysis simplified the transcriptome dynamics into two major gene modules and one unchanged gene expression baseline. Briefly, the natural logarithm of the measured level of a transcript $i$ at a specific time point $t, \ln X_{i}(t)$, is expressed as a sum of a logtransformed gene expression baseline, term $\ln X_{i}^{0}$, and several gene modules $\lambda_{j}(t) \times G_{i j}$, representing deviations from the common expression baseline. Each deviation term is a product of a time-dependent module score $\lambda_{j}(t)$, and the time-independent module-specific contribution score $G_{i j}$ of the gene $i$. Gene $i$ that displays large positive or negative contribution to a module $j$ (high positive or negative $\mathrm{G}_{i j}$ value) represents a gene that is functionally positively or negatively correlated with the module $j$. In other words, the biological function of the module $j$ could be inferred by functional enrichment analysis of genes with positive and negative $\mathrm{G}_{i j}$ values.

To implement surprisal analysis, we first computed the singular value decomposition (SVD) of the matrix $\ln \mathbf{X}(t)$. As described previously (Remacle et al., 2010), the SVD factored this matrix in a way that determined the two sets of parameters that are required in the surprisal analysis: the Lagrange multipliers $\left(\lambda_{j}(t)\right)$ for all gene modules at a given time point and for all times, as well as the module-specific contribution scores $\left(G_{i j}\right)$ for all transcripts $i$ at each gene module $j$. Further enrichment analysis of the functions associated with each module were performed based on the module-specific contribution scores of the genes associated with that module. The module-1 and module-2 scores of other published datasets on melanoma cell lines (Su et al., 2017) or melanoma patients' biospecimens (Hugo et al., 2015, 2016) were calculated as $\sum_{i}\left(\ln X_{i}\right) \cdot G_{i j}$ which considered the both the gene expression as well as the respective gene contribution towards each gene module.

Natural log-transformed transcriptome dataset and contributions from each gene module $\left(\lambda_{j}(t) G_{i j}\right)$ calculated from surprisal analysis were visualized using self-organized maps (SOMs). Here, the SOMs plotted individual sample as a single 2-dimensional heatmap and, at the same time, displayed high-resolution patterns. Thousands of input genes were assigned to 625 rectangular "tiles" (SOM nodes), each of which represented a mini-cluster of genes, arranged to form a pattern within a 2-dimensional mosaic map on the SOM grid. Each mini-cluster of genes was mapped onto the same tiles in each map, and the color of each tile represented the relative average expression of the gene mini-cluster within that tile. Most similar clusters were placed adjacent to 
each other in the mosaic map. Tiles at the same location represented the same group of genes across different conditions. Gene Expression Dynamics Inspector (GEDI) package was utilized to implement the SOM visualization (Eichler et al., 2003).

\section{Dynamic system modeling of two gene modules}

In order to more precisely infer the mathematical inter-regulation relationship between two gene modules, we performed dynamic systems modeling with regards to the average gene expression of the top 500 genes that have the highest positive or negative $\mathrm{G}$ values (weights). More specifically, for drug treatment condition, we have G1 positive genes and G1 negative genes (genes that are positive or negatively correlated with $\mathrm{M}_{\mathrm{late}}$ ), which are paired with $\mathrm{G} 2$ positive and $\mathrm{G} 2$ negative genes (genes that are positive or negatively correlated with $\mathrm{M}_{\text {early }}$ ) respectively. Therefore, we have 4 different scenarios for drug treatment condition. Similarly, we also have 4 different scenarios for the drug removal condition.

We started with a system of first-order mass equations that can consider all possible interaction relationships between the two modules. This system initially included terms for baseline, constant basal regulation $\left(\mathrm{B}_{1}\right.$ and $\left.\mathrm{B}_{\mathrm{e}}\right)$ on each gene module, first-order autoregulation from itself $\left(\mathrm{M}_{\mathrm{e}-\mathrm{e}}\right.$ and $\mathrm{M}_{1-1}$ ), and first-order regulation by the genes from the other module $\left(\mathrm{M}_{\mathrm{e}-1}\right.$ and $\left.\mathrm{M}_{1-\mathrm{e}}\right)$. We simultaneously fitted all coefficients through unbiased search using Markov Chain Monte Carlo (MCMC) in Python 3.0 and Gaussian distribution of coefficient probability. Initial coefficients were set on random uniform distributions. We also constrained coefficients such that the resulting fit would not lead to artificial oscillations with a frequency beyond the Nyquist frequency of our experimental sampling. Using the fitted parameters, our simulated trajectories of module1 ( $\left.\mathrm{M}_{\text {late }}\right)$ and module2 $\left(\mathrm{M}_{\mathrm{early}}\right)$ can recapitulate the original experimental data.

\section{Gene set enrichment analysis}

Gene Set Enrichment Analysis (GSEA) was conducted based on GSEA v2.2.3 software with 1000 permutations and weighted enrichment statistics. Normalized enrichment score (NES) was assessed across the curated Molecular-Signatures Database (MSigDB) Hallmark, C2 curated gene sets, and MITF signature (Hoek et al., 2008). To calculate the single-sample gene set enrichment, we used the GSVA program (Hänzelmann et al., 2013) to derive the absolute enrichment scores of previously experimentally validated gene signatures. The normalized $\log 2$ RPKM values were utilized as input for GSVA in the RNA-seq mode. The patient transcriptomic data was based on the patient transcriptomic data was obtained from (Kwong et al., 2015). GSEA v3.0 was used with the same parameters described above. 


\section{Transcription factor target and motif enrichment analysis}

Two different approaches were utilized to identify driving transcription factor in module2 ( $\left.\mathrm{M}_{\text {early }}\right)$ process. For the first approach, we filtered the TF that are associated with module2 (Pearson correlation with module 2 amplitude ( 22$)$ bigger than 0.8 or less than -0.8 ) and define them as module2 associated TFs. We then acquired the downstream targets genes for all module2 associated TFs using public database TFtargets (https://github.com/slowkow/tftargets). The KDM5B gene targets were manually verified by ChIP-seq data (GSE101045). We then further filter the module2-associated TFs based on the overlap of their downstream target gene with certain module1 ( $\left.\mathrm{M}_{\text {late }}\right)$. More specifically, for a certain TF in module2 ( $\left.\mathrm{M}_{\text {early }}\right)$, if its' downstreamtargeted genes are over-represented in module1 $\left(\mathrm{M}_{\text {late }}\right)$ process (Hypergeometric test with Bonferroni correction, FDR $<=0.05$ ), then this $\mathrm{TF}$ is selected as a candidate for driving TF in module2. For the second approach, we use HOMER to find enriched motifs in the promoter sequence of module 1 associated gene set (Pearson correlation with module2 amplitude ( $\lambda 2$ ) bigger than 0.8 or less than -0.8 ) with following parameters: -len 6,8,10,12 -start -1800 -end 100 -b -mset vertebrates. Then, we infer the potential TFs based on the enriched motif information.

\section{GDSC data analysis}

Cell lines from skin cutaneous melanoma (SKCM) samples, containing the $B R A F^{\mathrm{V} 600 \mathrm{E}}$ genetic mutation from the GDSC project, were selected to analyze the association between the transcriptional states before drug treatment and their drug responses (Iorio et al., 2016). Gene expression levels of the selected cell lines were projected to two gene modules ( $\mathrm{M}_{\text {early }}$ and $\mathrm{M}_{\text {late}}$ ). BRAF inhibitors Dabrafenib is selected to analyze the association of transcriptome state and drug response. Pearson correlation analysis and linear regression modeling for the two gene modules and the log-transformed IC50 values (Drug concentration that reduces viability by 50\%) or AUC (area under the dose-response curve) values were carried out.

\section{CCLE and TCGA analysis}

GSVA analysis was utilized to analyze the pathway enrichment scores across RNA-seq data of melanoma patient from TCGA database (Akbani et al., 2015) and from melanoma cell line data from CCLE (Barretina et al., 2012) database. The enrichment score of $\mathrm{M}_{\text {early }}$ and $\mathrm{M}_{\text {late }}$ associated genesets across all samples within the (patient or cell line) dataset were utilized as input to calculate pairwise Pearson correlations, and the average Pearson values of all possible pairwise correlations are used as co-occurrence score of $\mathrm{M}_{\text {early }}$ and $\mathrm{M}_{\text {late }}$ genesets within each dataset. 


\section{Patient data analysis}

Paired patient data before and after the MAPKi treatments were used to evaluate relevant gene expression levels and gene sets enrichment. These data were collected from two published papers. The gene expression levels and associated patient identification numbers in the original papers were provided in Table S6. Patient survival analysis was performed with high expression vs. low expression of selected genes from the TCGA melanoma (SKCM) data set including all stage III and IV patients. Kaplan-Meier method was used to estimate the survival rate, along with a logrank statistical test comparing the survival distribution. All tests were two-sided, and $\mathrm{p}$ values less than 0.05 were considered statistically significant.

\section{DATA AND SOFTWARE AVAILABILITY}

The accession number for the gene expression, ATAC-seq and ChIP-seq data reported in this paper is GEO: GSE134459. 

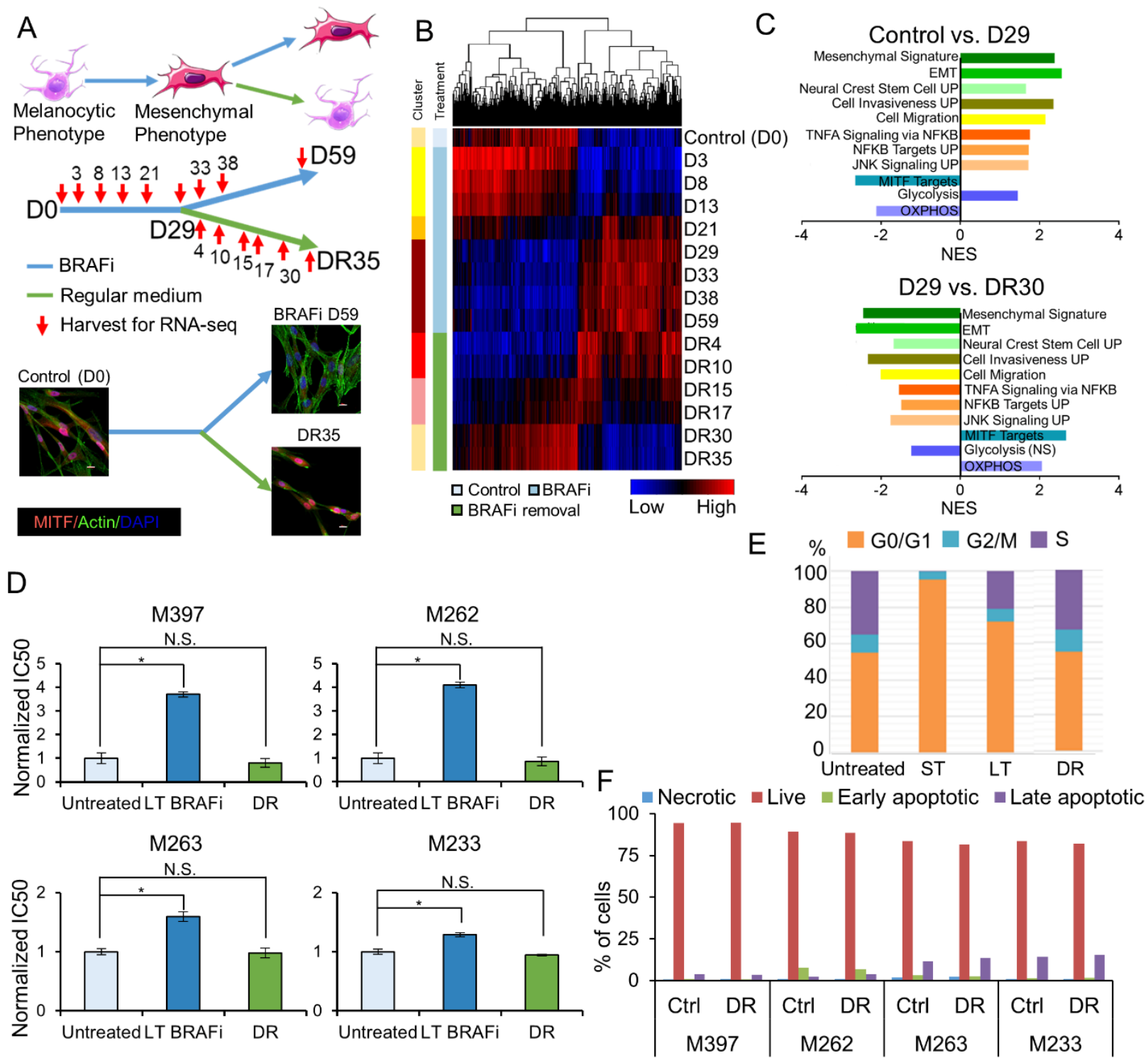

Figure 1. Adaptive drug resistance and reversibility across a panel of melanoma cell lines. (A) An illustration of the melanocyte-to-mesenchymal transition and the experimental timeline. Cells were treated with BRAF inhibitor (BRAFi) vemurafenib for 29 days (D29). BRAFi treatment continued for some cells up to D59, while other cells were followed over a 35 day period of drug removal (DR35). Cells were harvested for RNA-seq at the time points specified. (B) Heatmap of differential expressed genes (DEGs) at a series of time points of drug treatment and drug removal for M397 cells. Sidebars denote consensus clustering results of the variated genes from the samples (6 clusters) and their treatment conditions. DR30 and DR35 fall into the same cluster with the control sample. (C) Enriched molecular signatures associated with the adaptive transition in M397 cells. (D) Increased drug tolerance and reversed drug sensitivity across multiple melanoma cell lines with varying baseline sensitivities to BRAF inhibition evaluated by IC50 values of vemurafenib. LT: long-term; DR: drug release. Mean $\pm \mathrm{SD}$. (E) Cell cycle distribution across the reversible transition of M397 cells. (F) Cell viability and apoptotic profiles of untreated cells (Ctrl) 
bioRxiv preprint first posted online Aug. 5, 2019; doi: http://dx.doi.org/10.1101/724740. The copyright holder for this preprint (which

was not peer-reviewed) is the author/funder, who has granted bioRxiv a license to display the preprint in perpetuity.

All rights reserved. No reuse allowed without permission.

and reverted cells (upon 30 days drug removal) after 3-day BRAFi exposure. DR: drug removal. See also Figure S1 and Table S1 
A

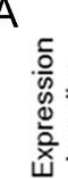
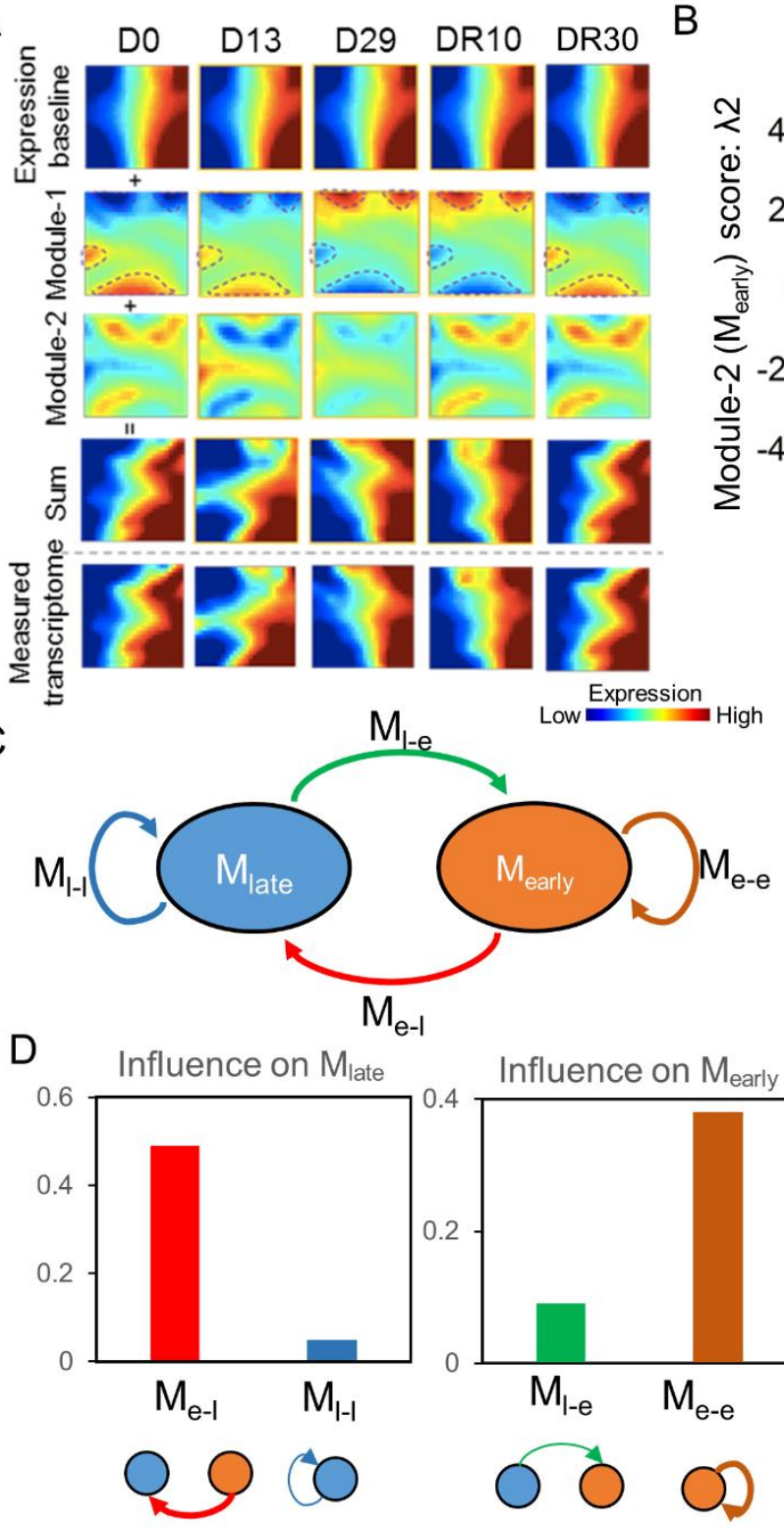

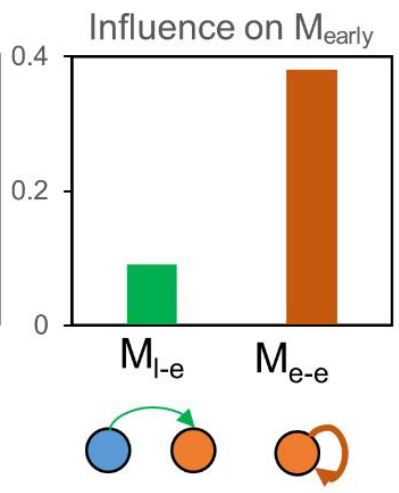

Module-1 $\left(\mathrm{M}_{\mathrm{lato}}\right)$ score: $\lambda 1$

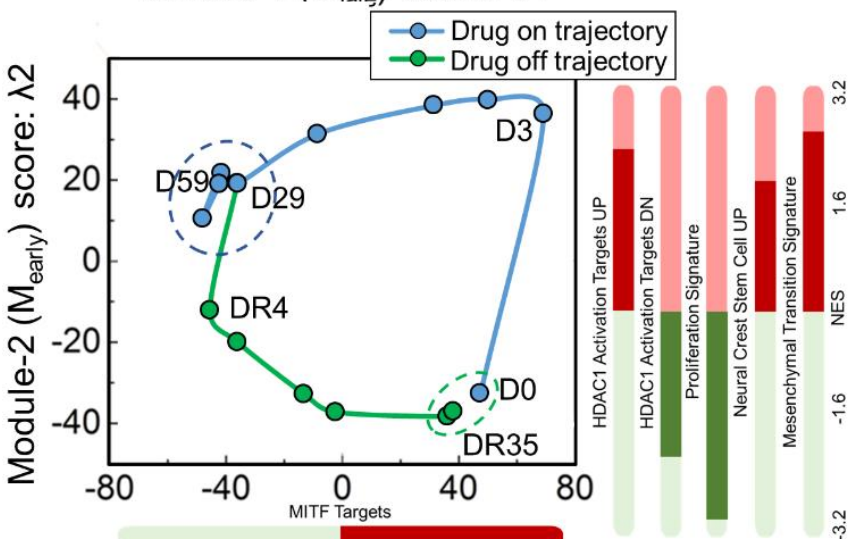

Mesenchymal Transition Signature

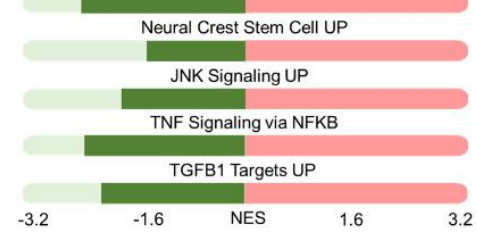

$E \cdot M_{\text {early }}$ genes (measured) $-M_{\text {early }}$ genes (predicted)

- $\mathrm{M}_{\text {later }}$ genes (measured) $-\mathrm{M}_{\text {late }}$ genes (predicted)

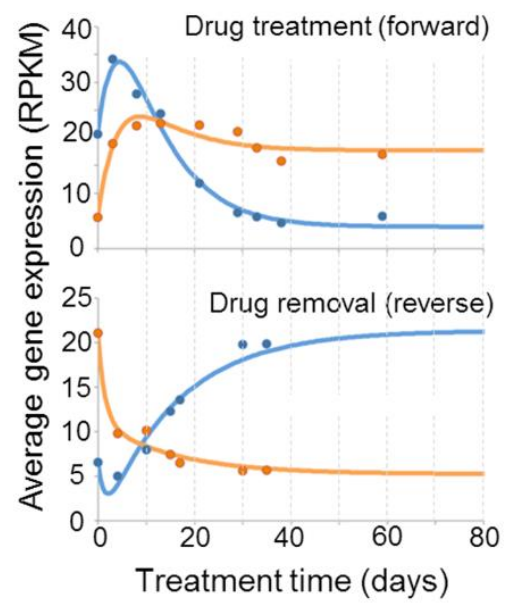

Figure 2. Information theoretic analysis and dynamic systems modeling of the reversible adaptive transition in M397 cells. (A) Application of surprisal analysis to the time-series transcriptome data over the transition. The transcriptome data, decomposed into a time invariant gene expression baseline plus two time-dependent gene modules, are illustrated as self-organizing maps (SOMs). Adding the baseline and first 2 gene modules recapitulates the experimentally measured transcriptome profiles. (B) The cyclic trajectory of the reversible transition plotted in the landscape defined by the first two gene modules. The blue and green dash lines circled the milieu of the mesenchymal-like drug-resistant state and drug naïve state, respectively. Selected enriched molecular processes (nominal $\mathrm{p}<0.05$ ) associated with each gene module are listed. NES: normalized enrichment score. (C) Schematic illustration of the simplified model for two gene module interactions. (D) The module-module interaction coefficient in the ordinary differential equations (ODEs) determined by fitting the model to the average expression level of the top 500 
genes associated with each gene module. (E) Experimentally measured and ODE fitted average expression levels of genes associated with the two modules in the forward and reverse directions of the cyclic transition. See also Figures S2, S3 and Tables S2, S3, S4. 


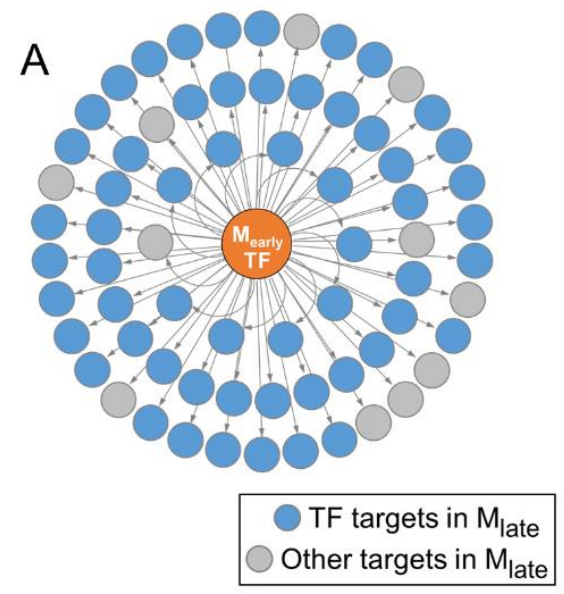

\section{B i. Target effector gene enrichment}

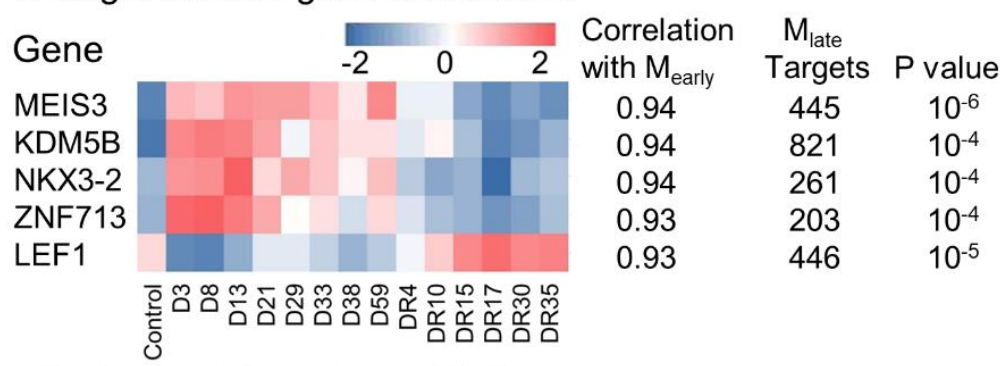

ii. cis-regulatory element inference
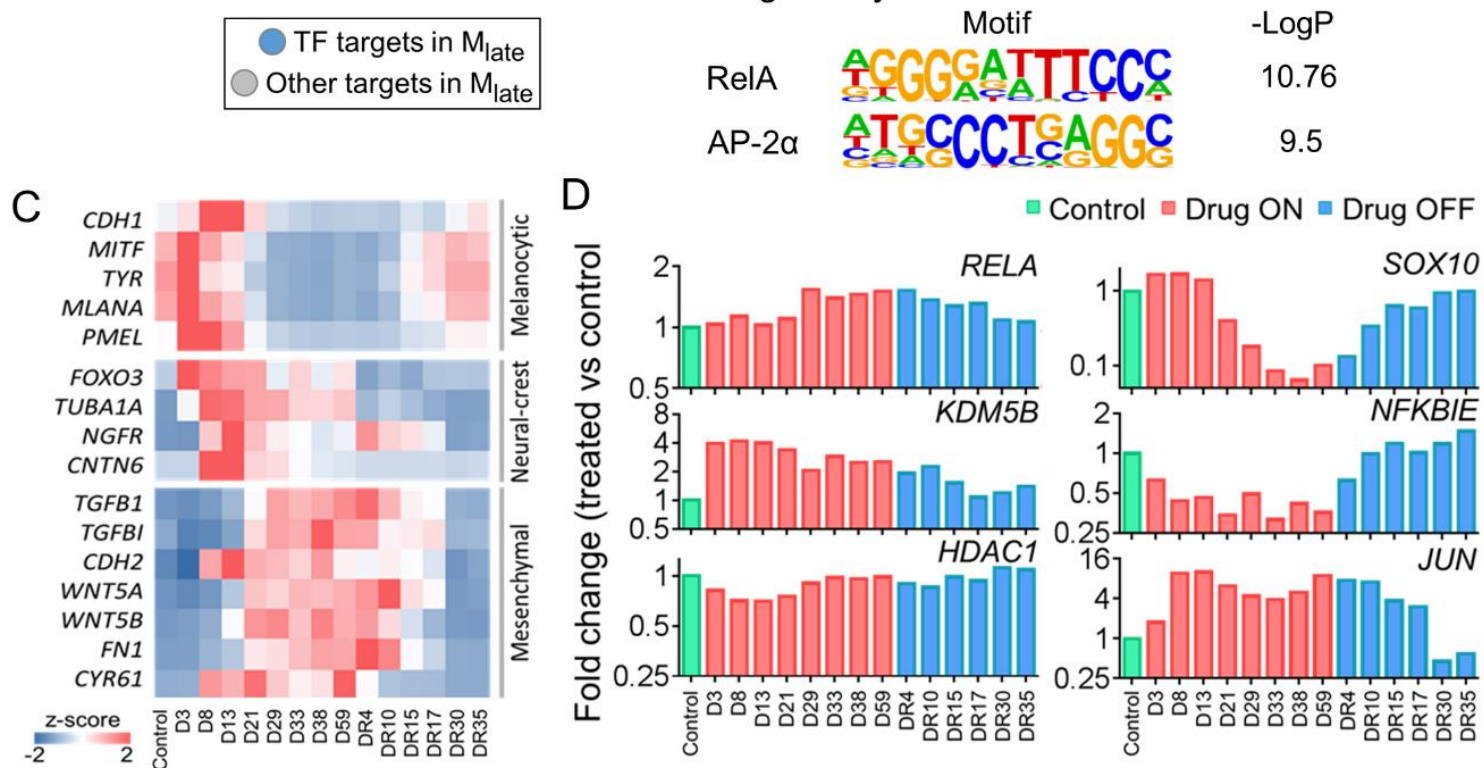

Figure 3. Bioinformatic inference of the critical regulators that drive the initiation of the reversible adaptive transition. (A) A scheme showing the target gene inferences. (B) Bioinformatic inferences based on the dynamic relationships between the two gene modules. (i) The list of enriched transcription factors (TF) and co-factors from target gene enrichment are ranked according to their absolute correlation coefficients with $\mathrm{M}_{\text {early }}$ scores with relative expression levels (z-score) shown as a heatmap. The target gene number and statistical significance ( $p$ values) for each enriched element are listed to the right. (ii) Enriched motifs from the cisregulatory elements of genes highly correlated $(\rho>0.8)$ with $\mathrm{M}_{\text {late. }}$ Top two significantly enriched motifs are listed. $-\log 2 p$ values are shown to the right. (C) Relative expression levels of cell-state specific genes over the course of the adaptive cyclic transition (D) Gene expression levels, normalized to D0, of the critical TFs/co-factors involved in the adaptive transition. See also Figure S4 

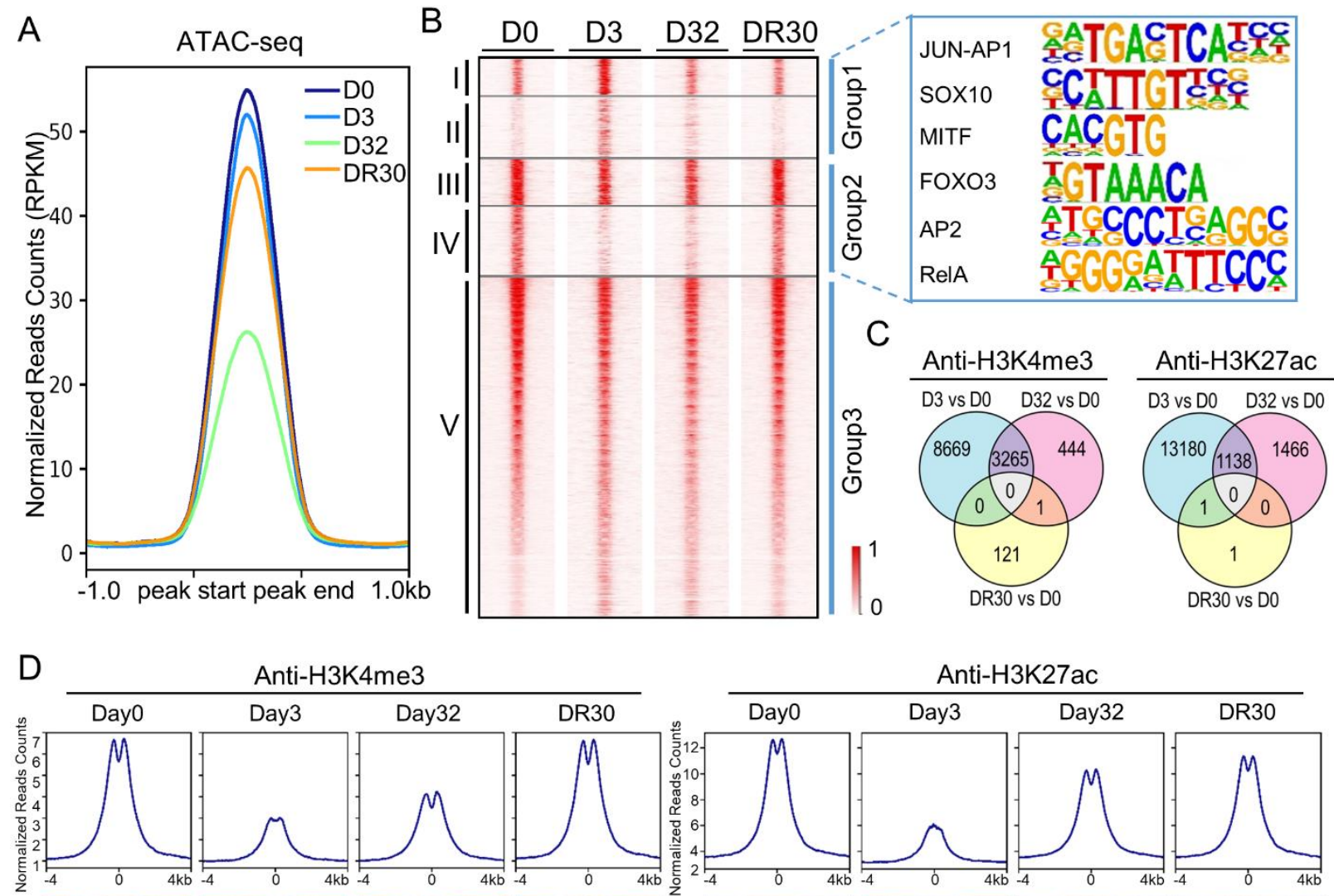

Anti-H3K27ac
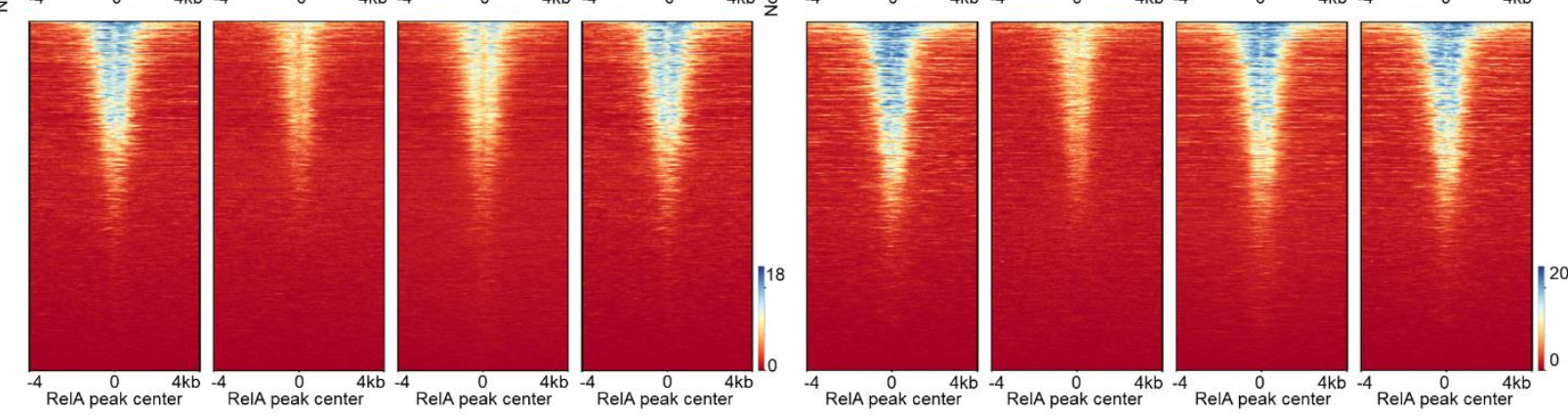

Figure 4. Epigenetic reversibility of the adaptive transition. (A) Chromatin accessibility change assessed by average peak signal of ATAC-seq across the entire genome. The $\mathrm{x}$-axis shows flanking regions of $+/-1 \mathrm{~kb}$ around the peak center. (B) Heatmap of chromatin accessibility changes assessed by average ATAC-seq peak signal across all peaks, at selected time points over the transition. K-mean clustering of rows identifies five chromatin regions that grouped into D3 enriched (group 1), D0/DR30 enriched (group 2), and independent of drug treatment (group 3 ). Color corresponds to the normalized ATAC-seq signal. Relevant transcription factor binding motifs are indicated for cluster groups 1 and 2. (C) Venn diagrams showing the numbers and overlaps of differential ChIP-seq peaks for H3K4me3 and H3K27ac. Each circle represents changes in those peaks between two time points, while the intersection of the circles represent changes that are shared between circles. (D) ChIP-seq profile plots show the average H3K4me3 (left) and H3K27ac (right) ChIP-seq signal across peaks identified by RelA ChIP-seq, with 
bioRxiv preprint first posted online Aug. 5, 2019; doi: http://dx.doi.org/10.1101/724740. The copyright holder for this preprint (which was not peer-reviewed) is the author/funder, who has granted bioRxiv a license to display the preprint in perpetuity.

All rights reserved. No reuse allowed without permission.

heatmap representation of each peak shown below. The $\mathrm{x}$-axis shows flanking regions of $+/-4 \mathrm{~kb}$ around the peak center. See also Figure S5 and Table S5. 
bioRxiv preprint first posted online Aug. 5, 2019; doi: http://dx.doi.org/10.1101/724740. The copyright holder for this preprint (which was not peer-reviewed) is the author/funder, who has granted bioRxiv a license to display the preprint in perpetuity.

All rights reserved. No reuse allowed without permission.

A

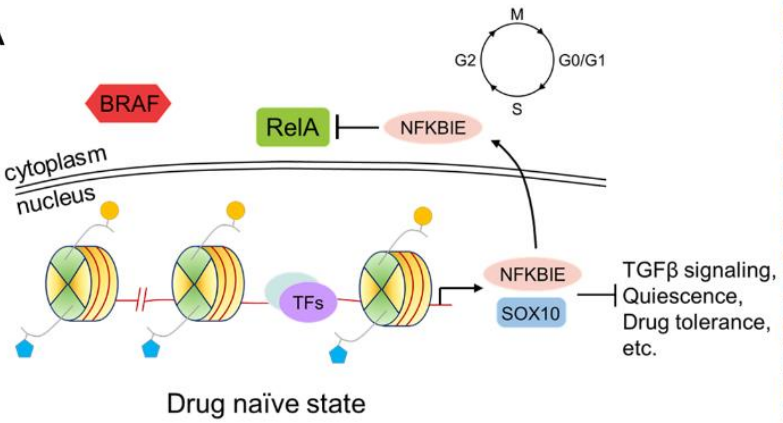

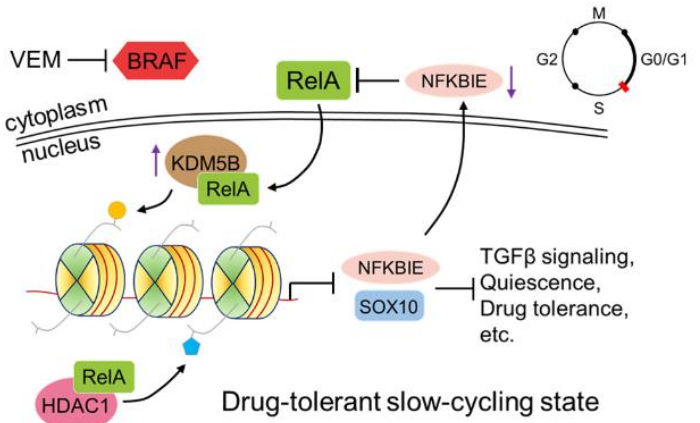

Drug-tolerant slow-cycling state
B
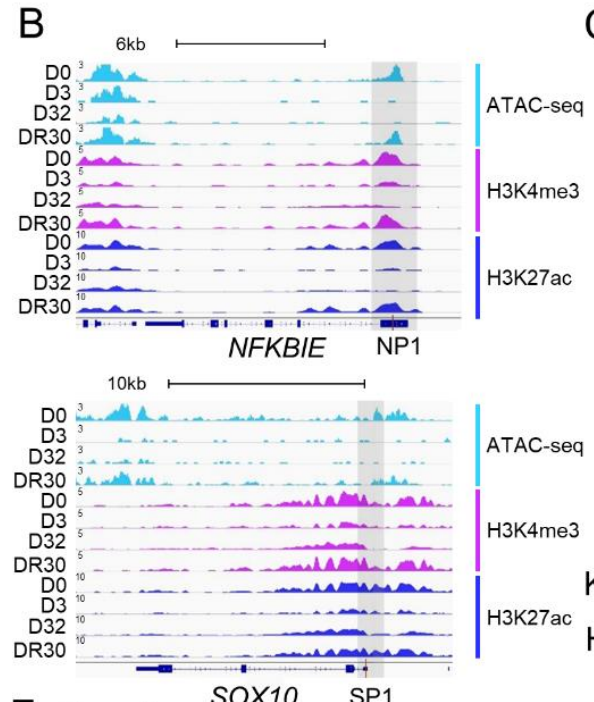

F
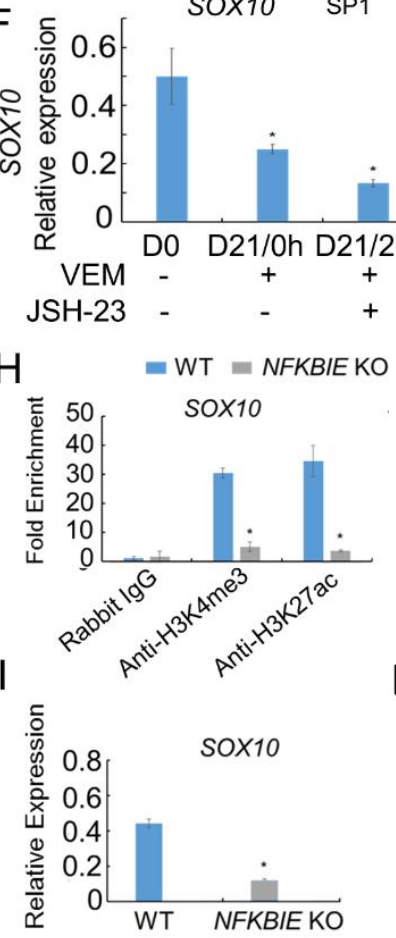

C
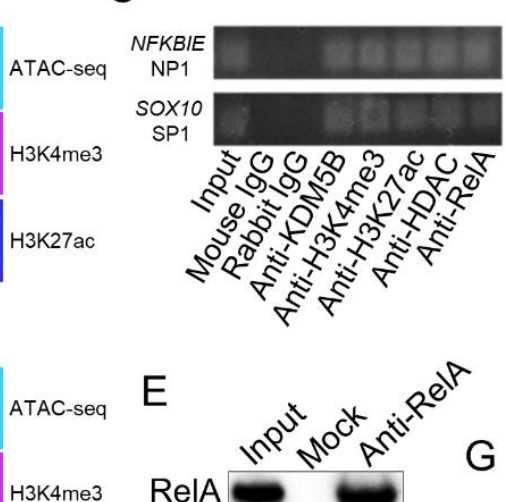

G

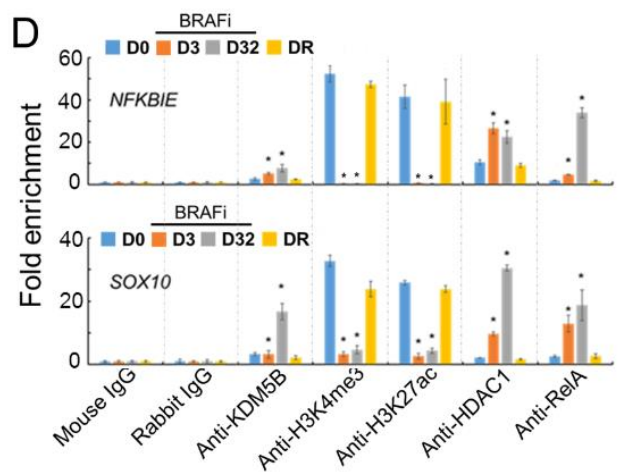

${ }^{\text {H3K27ac }}$ HDAC1

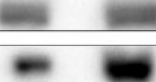

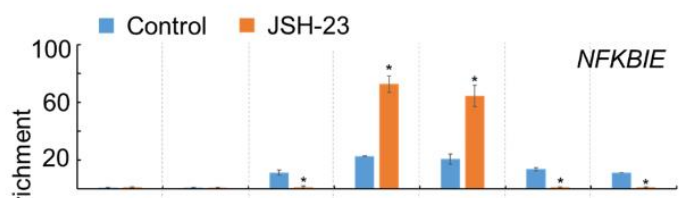
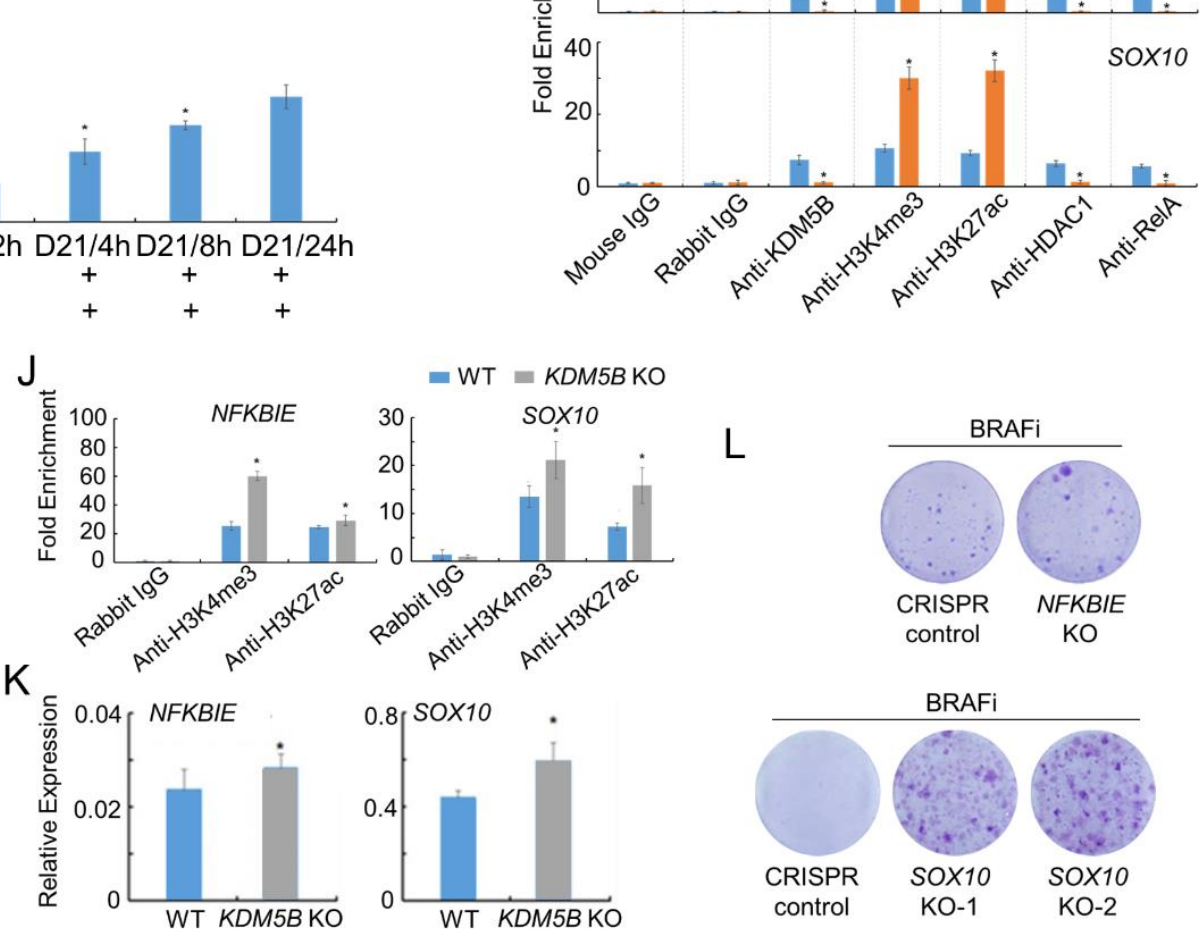

L
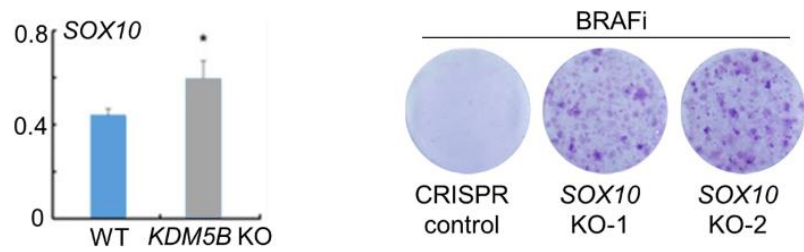

Figure 5 Molecular mechanism that underlies the reversible adaptive transition. (A) 
Illustration of the mechanism of epigenetic regulation before and after BRAF inhibition. Left panel: retention of RelA in the cytoplasm and open chromatin at the promoter regions of SOX10 and NFKBIE. Right panel: BRAFi induces translocation of RelA into the nucleus, allowing RelA to recruit histone modifiers $\mathrm{KDM} 5 \mathrm{~B}$ and $\mathrm{HDAC1}$ to the target genes to reduce chromatin accessibility and epigenetically repress of SOX10 and NFKBIE expression. Functional consequences, such as increased TGF $\beta$ signaling and adaptive drug resistance, result in turn. The illustrations of the cell cycle reflect the measured cell cycle arrest observed upon short-term BRAF inhibition. (B) ATAC-seq and ChIP-seq profiles at the promoter regions of NFKBIE and SOX10, at selected time points across the reversible transition. (C) ChIP-PCR data illustrates the binding and co-localization of RelA, KDM5B, HDAC1, H3K4me3, and H3K27ac to promoter regions of SOX10 and NFKBIE (labeled NP1 and SP1, respectively). (D) ChIP-qPCR assessment of the binding profiles of RelA, KDM5B, HDAC1, H3K4me3, and H3K27ac on the promoter regions of NFKBIE and SOX10 at a series of time points across the reversible transition. (E) Coimmunoprecipitation of RelA with KDM5B and HDAC1, confirming the binding between RelA and the two histone modifiers $(* \mathrm{P}<0.05$ compared to respective $\mathrm{D} 0)$. (F) The recovery of SOX10 gene expression levels of M397 cells pretreated with BRAFi for 21 days (D21), and then co-treated for 0-24 hours with BRAFi and JSH-23 (*P<0.05 compared to D0) (G) ChIP-qPCR assessment of the binding profiles of RelA, KDM5B, HDAC1, H3K4me3, and $\mathrm{H} 3 \mathrm{~K} 27 \mathrm{ac}$ on the prompter regions of NFKBIE and SOX10 for control and JSH-23 24h-treated cells ( $* \mathrm{P}<0.05$ compared to respective control). (H) ChIP-qPCR of the binding profiles of H3K4me3 and H3K27ac on the promoter region of SOX10 from NFKBIE KO M397 cells ( $* \mathrm{P}<0.05$ compared to respective WT). (I) SOX10 expression levels after NFKBIE KO compared to wild type (WT) $(* \mathrm{P}<0.05$ compared to WT) (J) ChIP-qPCR of the binding profiles of $\mathrm{H} 3 \mathrm{~K} 4 \mathrm{me} 3$ and $\mathrm{H} 3 \mathrm{~K} 27 \mathrm{ac}$ on the promoter regions of NFKBIE and SOX10 from KDM5B KO M397 cells (*P<0.05 compared to WT) (K) Expression levels of $S O X 10$ and NFKBIE after KDM5B KO compared to WT (*P<0.05 compared to WT). (L) Clonogenic assays of NFKBIE KO or $S O X 10 \mathrm{KO}$ cells related to respective controls. 
bioRxiv preprint first posted online Aug. 5, 2019; doi: http://dx.doi.org/10.1101/724740. The copyright holder for this preprint (which was not peer-reviewed) is the author/funder, who has granted bioRxiv a license to display the preprint in perpetuity.

All rights reserved. No reuse allowed without permission.
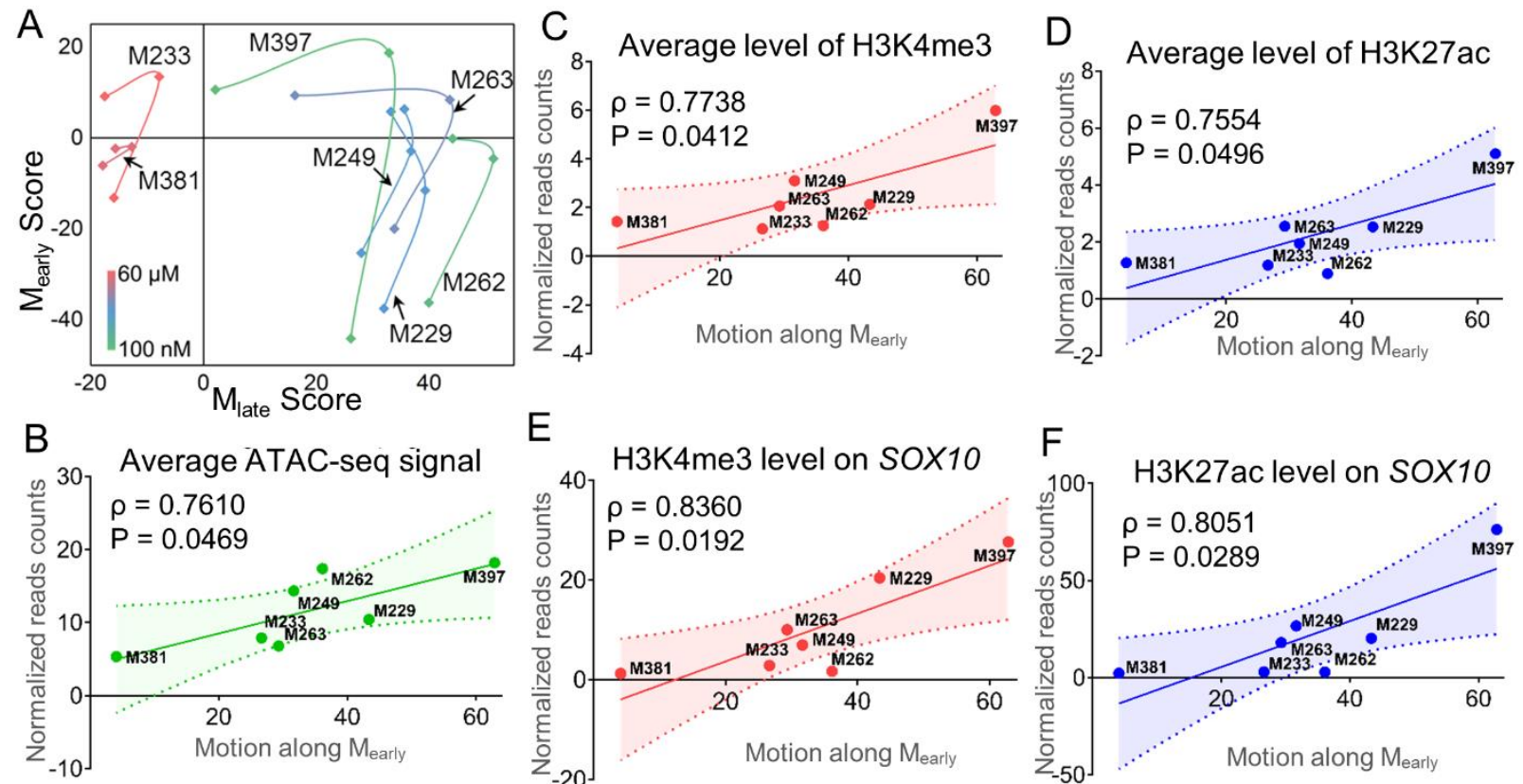

$\mathrm{E}$
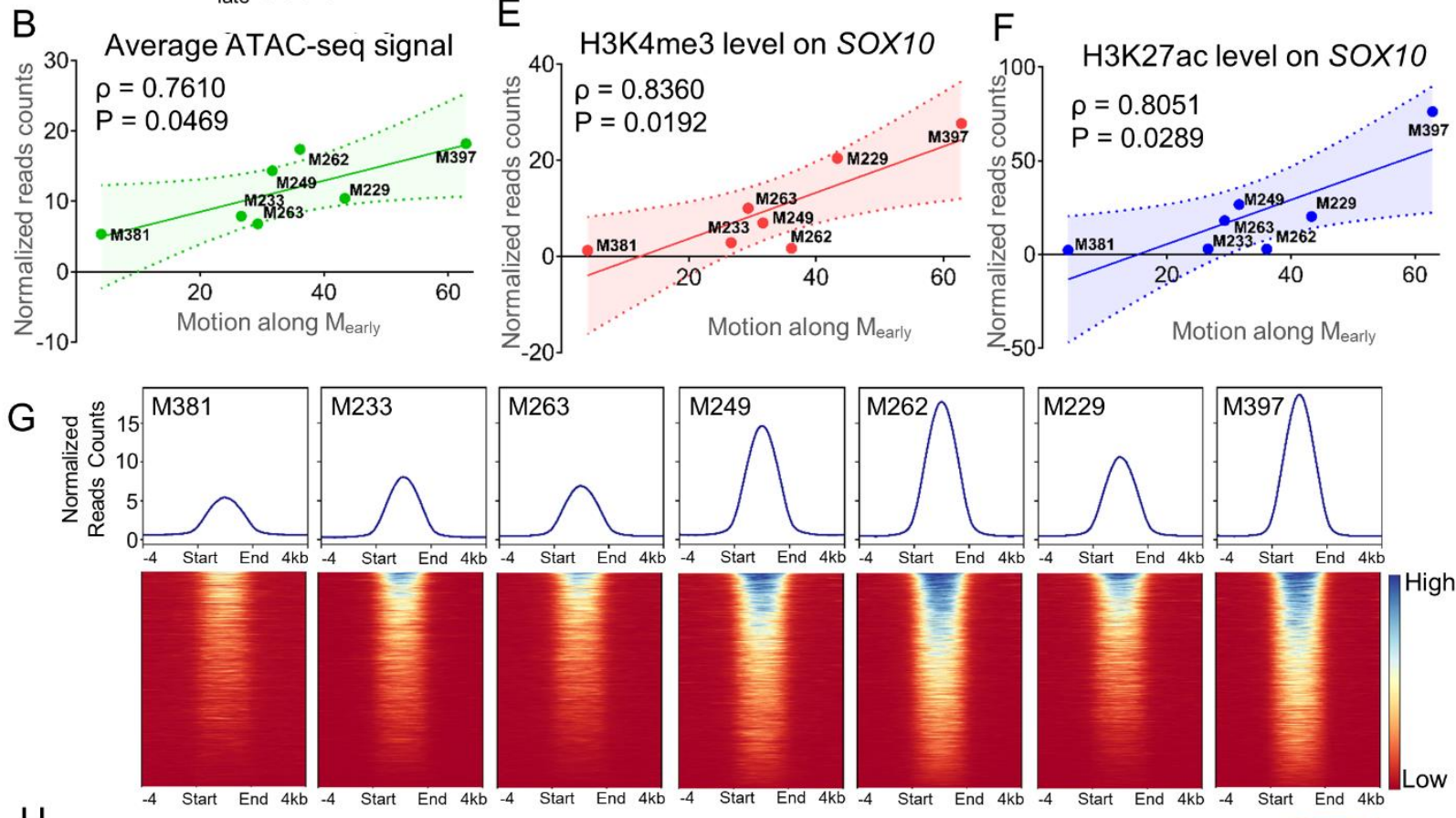

$\mathrm{H}$

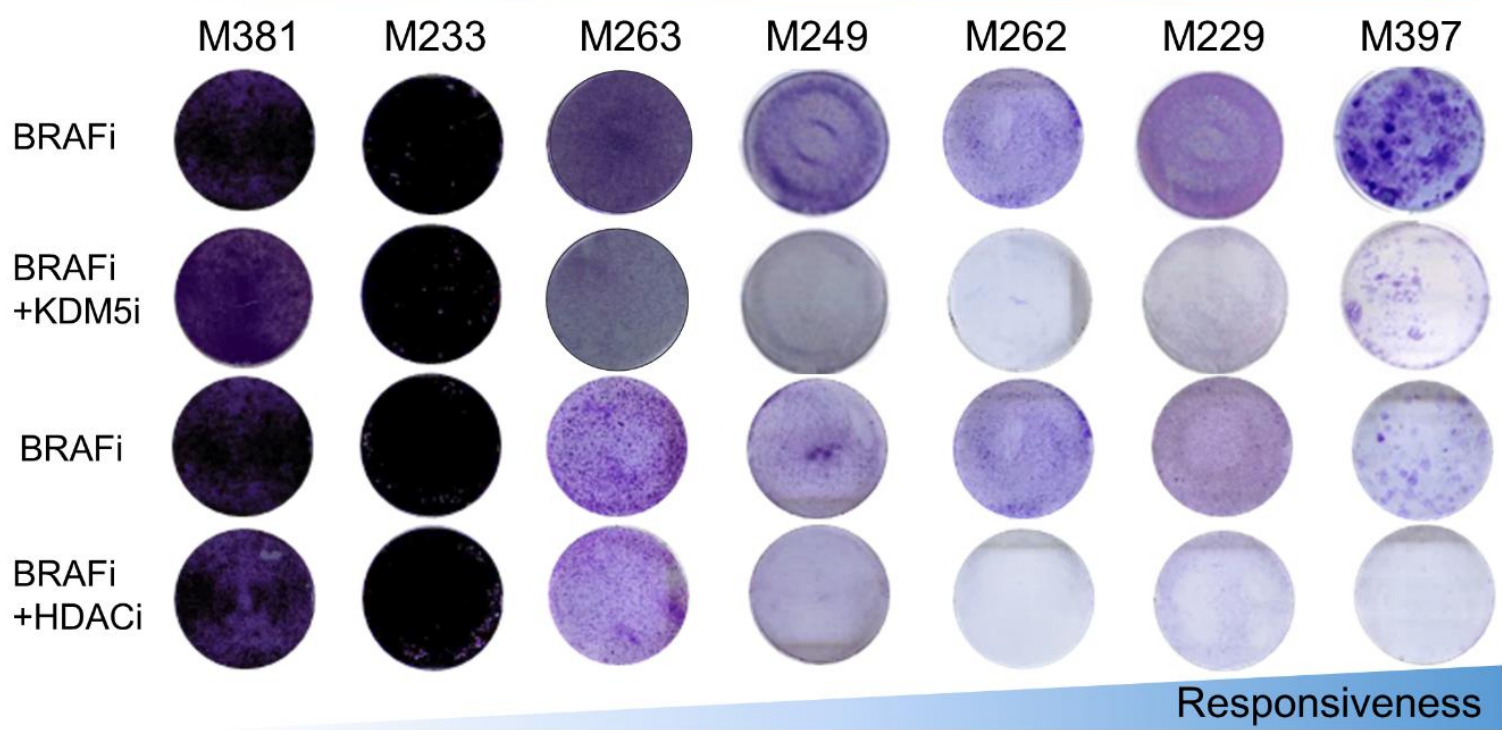

Figure 6. The generality and molecular underpinning of the phenotypic plasticity of patient- 
derived melanoma cell lines in response to BRAF inhibition. (A) Quantification of phenotypic plasticity upon BRAF inhibition across a panel of melanoma cell lines. The transcriptome data are projected to the 2D plane defined by the two gene modules ( $\mathrm{M}_{\text {early }}$ and $\left.\mathrm{M}_{\text {late }}\right)$ and connected by smooth lines. The data points denote 0-day (D0), D3, and D21 BRAFi treatment in counterclockwise order, respectively. The color of the line encodes the baseline IC50 value of the cell line. (B-F) The correlation between short-term plasticity (motion along $\mathrm{M}_{\text {early }}$ ) and the (B) average ATAC-seq signal, (C) average H3K4me3 (D) and H3K27ac ChIP-seq signal across all peaks, as well as (E) average H3K4me3 and (F) H3K27ac ChIP-seq signal on the transcription start site (TSS) region of SOX10 across all cell lines with Pearson correlation coefficients and pvalues as shown. The shaded regions of panels B-F denote $95 \%$ CIs of each linear fitting. (G) The chromatin accessibility of a panel of melanoma cell lines quantified by the average ATAC-seq signal across all peaks with heatmap view of each peak shown below. The $\mathrm{x}$-axis includes flanking regions of $+/-4 \mathrm{~kb}$ around the peak center. $(\mathrm{H})$ Clonogenic assays for BRAFi monotherapy and combination therapies simultaneously targeting the driver oncogene BRAF and histone modifiers KDM5B and HDAC1. The cell lines are ordered from left to right with increased plasticity. See also Figure S6. 

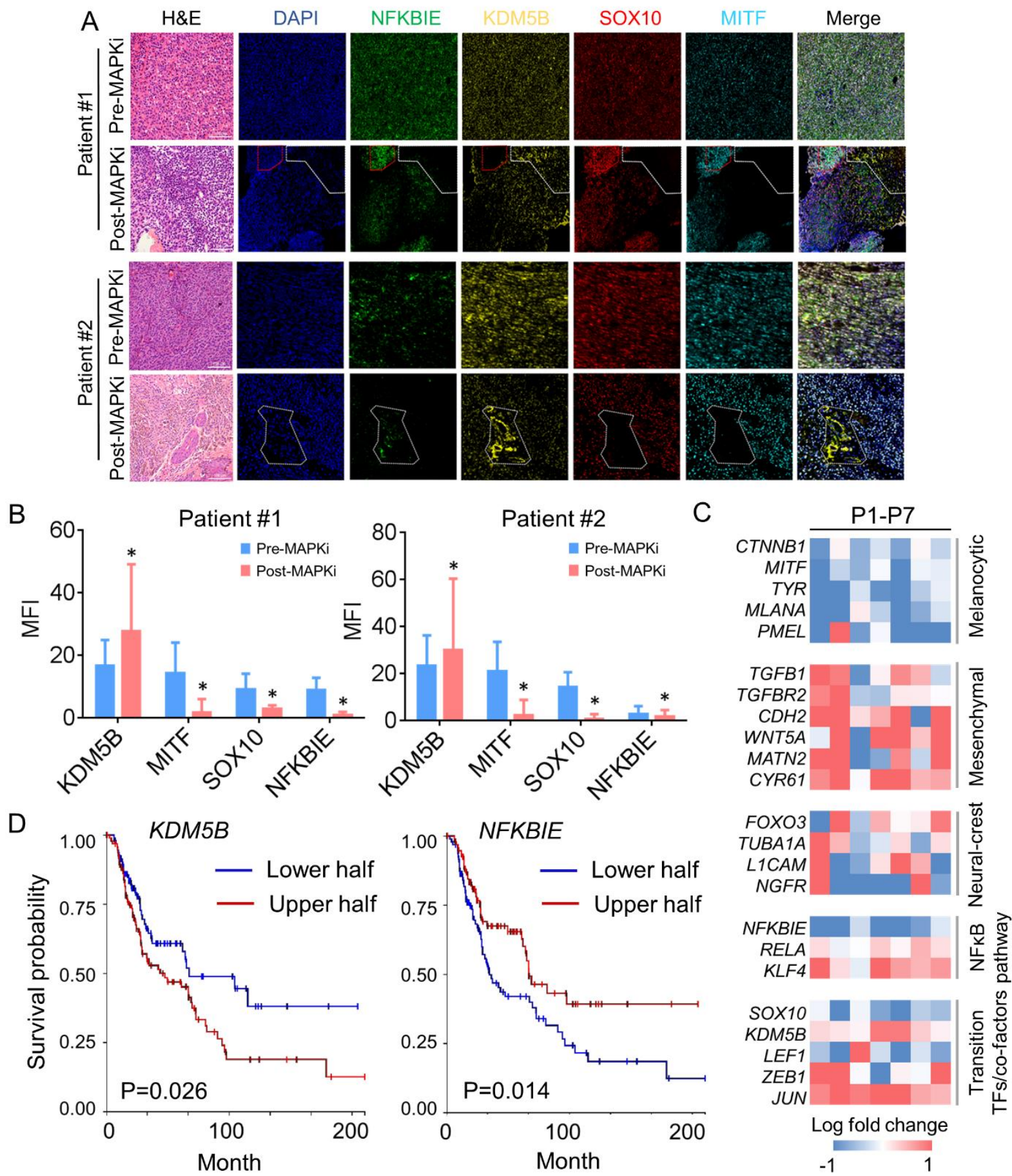

Figure 7. The clinical relevance of the drug-induced chromatin remodeling mechanism. (A) Immunohistochemical (IHC) staining of melanoma tissue biopsies from two patients bearing $B R A F$-mutant melanoma before and after MAPKi treatment. Hematoxylin and eosin (H\&E) staining shown in left column followed by a staining panel from pre-treatment or post-treatment biopsies. Post-treatment tissue was collected at the onset of tumor recurrence. The stains are DAPI nuclear stain (blue), NFKBIE (green), KDMB5 (yellow), SOX10 (red), MITF (cyan), and merge. The region highlighted by white dashed lines in the post-MAPKi tissues display reduced MITF, 
NFKBIE, and SOX10 expression and elevated KDM5B expression, consistent with the adaptive resistance mechanism. The region highlighted by red dashed lines retained the MITF, NFKBIE, and SOX10 expressions but with loss of KDM5B after treatment. (B) Digitized mean fluorescence intensity (MFI) of the areas highlighted by white dashed lines for the selected markers in two patients. Data are represented as mean $\pm \mathrm{SD}(* \mathrm{P}<0.05$ compared to respective pre-MAPKi). (C) Log-fold change in the expression of relevant genes (post-treatment vs baseline), collated from published datasets of BRAF-mutant melanoma patients treated with MAPK inhibitors (STAR Method). (D) Kaplan-Meier plots assembled using TCGA data sets of tumors from patients with stages III and IV melanomas with log-rank P values shown. See also Figure S7 and Table S6. 


\section{Supplemental Information for}

\section{Kinetic Inference Resolves Epigenetic Mechanism of Drug Resistance in Melanoma}

Yapeng Su, ${ }^{1,2,3,14}$ Xiang Lu, ${ }^{4,14}$ Guideng Li, ${ }^{3}$ Chunmei Liu, ${ }^{1}$ Yan Kong, ${ }^{5}$ Jihoon W. Lee, ${ }^{2}$ Rachel $\mathrm{Ng},{ }^{2}$ Stephanie Wong, ${ }^{3}$ Lidia Robert, ${ }^{6}$ Charles Warden, ${ }^{7}$ Victoria Liu, ${ }^{2}$ Jie Chen, ${ }^{8}$ Zhuo Wang, ${ }^{8}$ Yezi Yang, ${ }^{4}$ Hanjun Cheng, ${ }^{1}$ Alphonsus H. C. Ng, ${ }^{1}$ Guangrong Qin, ${ }^{1}$ Songming Peng, ${ }^{2}$ Min Xue, ${ }^{2}$ Dazy Johnson, ${ }^{4}$ Yu Xu, ${ }^{9}$ Jinhui Wang, ${ }^{7}$ Xiwei Wu,${ }^{7}$ Ilya Shmulevich, ${ }^{1}$ Qihui Shi,,${ }^{8,10}$ Raphael Levine, ${ }^{4,11}$ Antoni Ribas, ${ }^{4,6,12,13}$ David Baltimore, ${ }^{3}$ Jun Guo, ${ }^{5,15}$ James R. Heath, ${ }^{1,2,15, *}$ and Wei Wei ${ }^{1,4,13,15,16, *}$

*Correspondence: jheath@systemsbiology.org (J.R.H.), wwei@systemsbiology.org (W.W.) 
bioRxiv preprint first posted online Aug. 5, 2019; doi: http://dx.doi.org/10.1101/724740. The copyright holder for this preprint (which was not peer-reviewed) is the author/funder, who has granted bioRxiv a license to display the preprint in perpetuity.

All rights reserved. No reuse allowed without permission.

\section{Supplemental Figures}

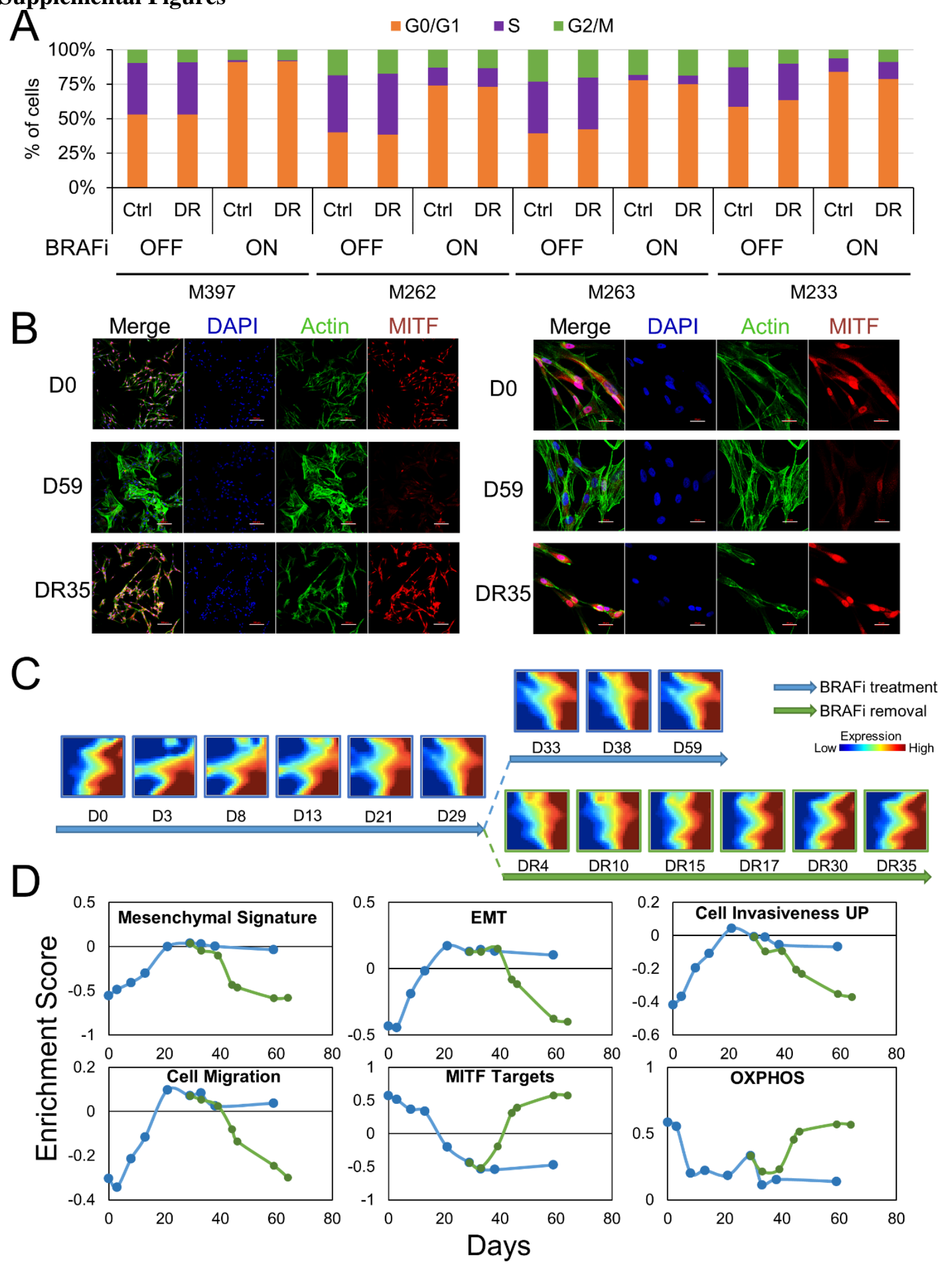

Figure S1. Reversible adaptive resistance across melanoma cell lines. Related to Figure 1. 
A. Stacked bar plot shows the fraction of cells viable in G0/G1, S, and G2/M phases (y-axis) for different melanoma cell lines. Each cell line has two different assay conditions: observed either before treatment, or after pretreated with BRAFi for 30 days and drug removal in normal medium for another 30 days. Cells in both conditions underwent cell cycle analysis at both drug-naïve condition and retreatment with BRAFi for another 3 days. Cells that have gone through drug treatment and drug removal have the same cell cycle distribution as cells that never receive drug treatment.

B. Immunostaining of M397 cells at different stages of reversible adaptive drug resistance. M397 cells before treatment (D0, first row), after treatment with BRAFi for 59 days (D59, second row), and pretreated with BRAFi for 29 days and then cultured with normal medium for another 35 days (DR35, third row) were used for immunostaining of MITF (red), actin (green), and DAPI (blue). The cell morphology at D0 is very similar to that at DR35. Cell morphology at D59 is very different from the ones at the other two conditions. Left panel scale bar 100um, right panel scale bar $20 \mathrm{um}$.

C. Two-dimensional self-organizing maps (SOMs) of overall transcriptome profiles of cells collected at different stages across the reversible adaptive drug response.

D. Enrichment scores of representative gene sets across different stages of the reversible adaptive response. Enrichment scores at different time points are shown as dots connected with solid lines. The scores stabilize after prolonged drug treatment (after 21 days of BRAFi, blue line) and return to the enrichment score of day 0 after long-term drug removal (green line). 


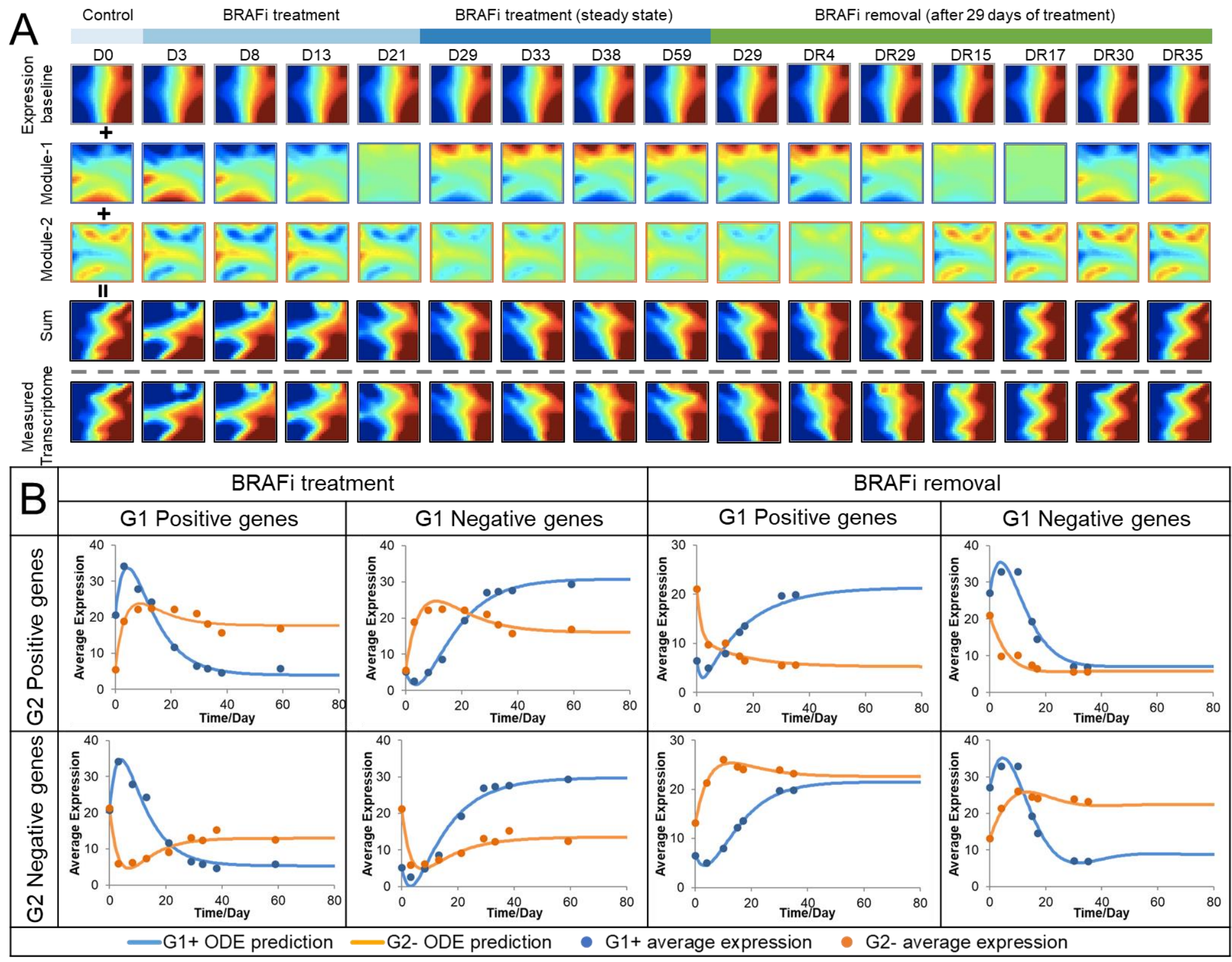

Figure S2. Surprisal analysis of the reversible adaptive transition in M397. Related to Figure 2. 
(A) Application of surprisal analysis to the kinetic transcriptome profile with respect to the reversible adaptive transition. The transcriptome data, decomposed into a time-invariant gene expression baseline plus two time-dependent gene modules, are illustrated as self-organizing maps (SOMs). Adding the expressions of the baseline gene module and time-dependent module-1 and module-2 recapitulates the experimentally measured transcriptome profiles visualized by the fact that the patterns of the SOMs in the last row (experimentally measured transcriptome profiles) are almost identical to those at second last row (predicted transcriptome profiles from surprisal analysis by adding first three rows).

(B) ODE predictions (smooth line) are consistent with the average expression levels of genes associated with module-1 and module2 from experimental measurements (dots) in the forward and reverse directions of the adaptive transition. More specifically, for

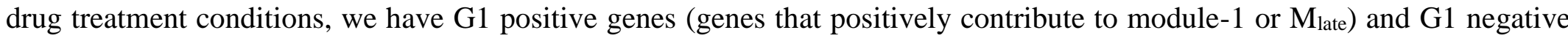
genes (genes that negatively contribute to module-1 or $\mathrm{M}_{\text {late }}$ ), which are paired with $\mathrm{G} 2$ positive and $\mathrm{G} 2$ negative genes (genes that positively and negatively contribute to module-2 or $\mathrm{M}_{\text {early }}$ ) respectively. Therefore, we have four different scenarios for drug treatment and four different scenarios for drug removal. 


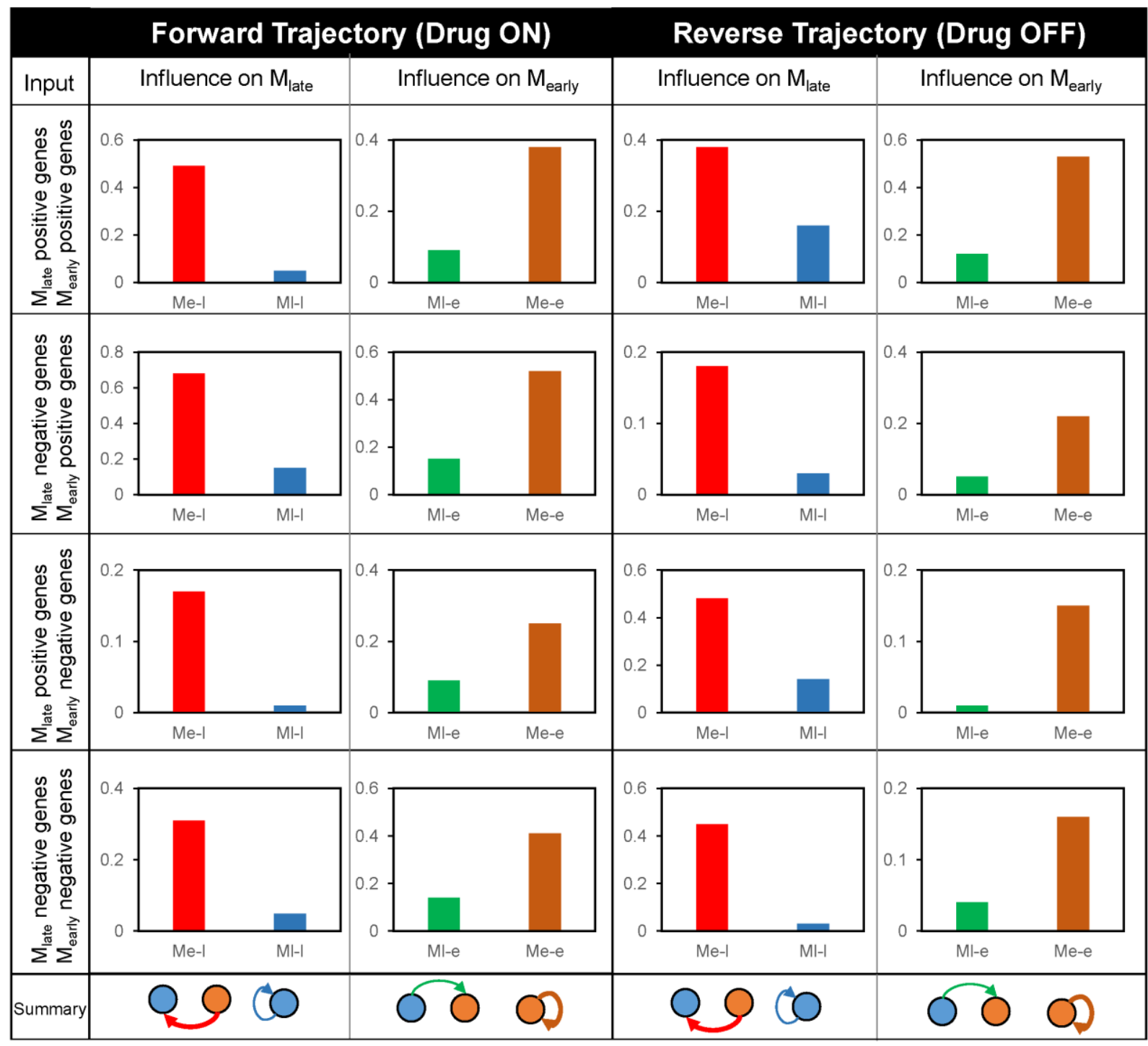

Figure S3. Best fitted parameters from dynamic system modeling of the reversible adaptive transition in M397. Related to Figure 2. The module-module interaction coefficients in the ordinary differential equations (ODEs) determined from fitting the ODE model to the average expression level of the top 500 genes associated with each gene module. More specifically, for drug treatment condition, we have G1 positive genes (genes that positively contribute to module1 or $\mathrm{M}_{\text {late }}$ ) and $\mathrm{G} 1$ negative genes (genes that negatively contribute to module-1 or $\mathrm{M}_{\text {late}}$ ), which are paired with G2 positive and G2 negative genes (genes that positively and negatively contribute to module-2 or $\mathrm{M}_{\text {early }}$ ), respectively. Therefore, we have four different scenarios for drug treatment. Similarly, we also have another four different scenarios for the drug removal. 


\section{A Strategy 1. Target effector gene inference:}

Find the TFs/co-factors correlated with $\mathrm{M}_{\text {early }}$ process, further filter for the ones whose target genes over-represented in the genes correlate with $\mathrm{M}_{\text {late }}$

TFs correlated with $\mathrm{M}_{\text {early }}$ scores with absolute correlation coefficient (CC) $\rho>0.9$

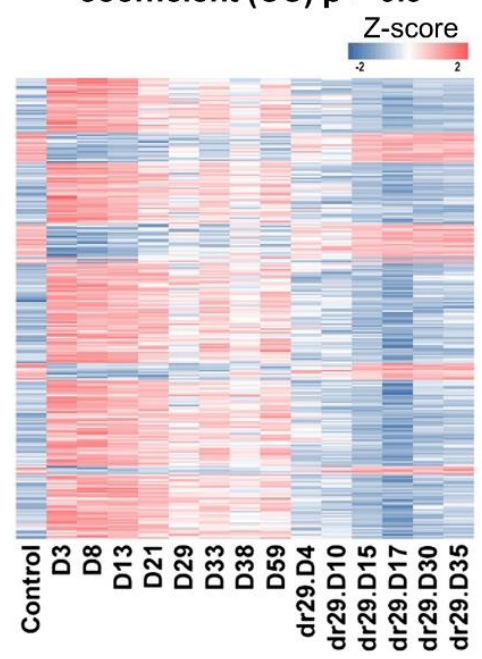

Identify targets of these TFs/cofactors using online database (TFtargets)
Quantify the target overlap with genes correlated with $M_{\text {late }}$ process

(absolute $\mathrm{CC} \rho>0.8$ )

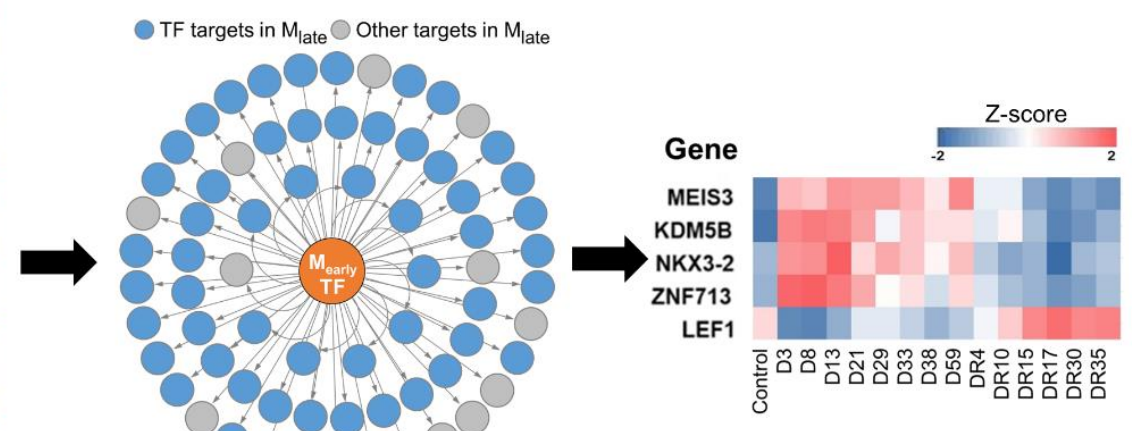

Inferred top driving TFs

B Strategy 2. cis-regulatory element inference:

Identify the common motifs from $\mathrm{M}_{\text {late }}$ gene promoters, and then infer the TFs that bind to those motifs and thus regulate the $\mathrm{M}_{\text {late }}$ genes.

Genes correlated with $\mathrm{M}_{\text {late }}$ scores

( absolute $\mathrm{CC} \rho>0.8$ )

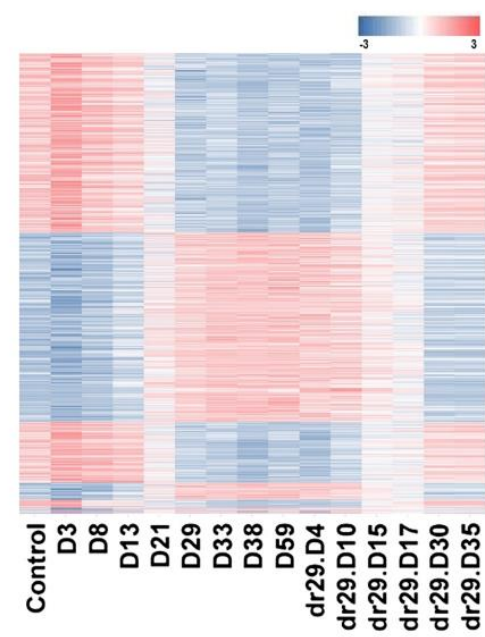

Enrich common motifs for gene promoters

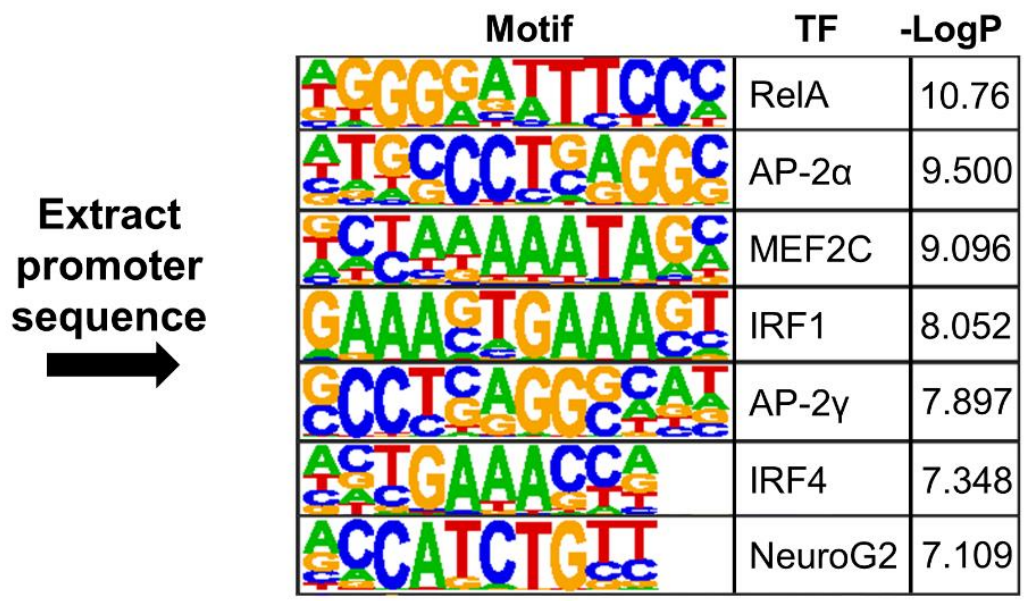

Figure S4. Strategies for inference of the critical regulators that drive the initiation of the reversible adaptive transition. Related to Figure 3.

(A) Target gene inference based on the dynamic relationships between the two gene modules. The TFs/co-factors whose expression kinetics are correlated with the module scores of 
$\mathrm{M}_{\text {early }}$ (left panel) are mapped to their target genes, followed by assessing the target gene enrichments in the genes correlated with $\mathbf{M}_{\text {late }}$ scores (middle panel). The inferred transition-driving TFs/co-factors whose target genes are significantly overrepresented in $\mathrm{M}_{\text {late }}$ are ranked by their absolute correlation coefficients with $\mathrm{M}_{\text {early }}$ scores. The top-5 elements were listed.

(B) Common motif inference to extract enriched motifs from genes highly correlated with $\mathrm{M}_{\text {late }}$ (left panel) and to identify the TFs that bind to these motifs and regulate cell state regression (right panel). Seven significantly enriched motifs with $\mathrm{p}<0.01$ (i.e. $-\log _{2} \mathrm{P}>6.64$ ) were identified and listed. 
bioRxiv preprint first posted online Aug. 5, 2019; doi: http://dx.doi.org/10.1101/724740. The copyright holder for this preprint (which was not peer-reviewed) is the author/funder, who has granted bioRxiv a license to display the preprint in perpetuity.

All rights reserved. No reuse allowed without permission.
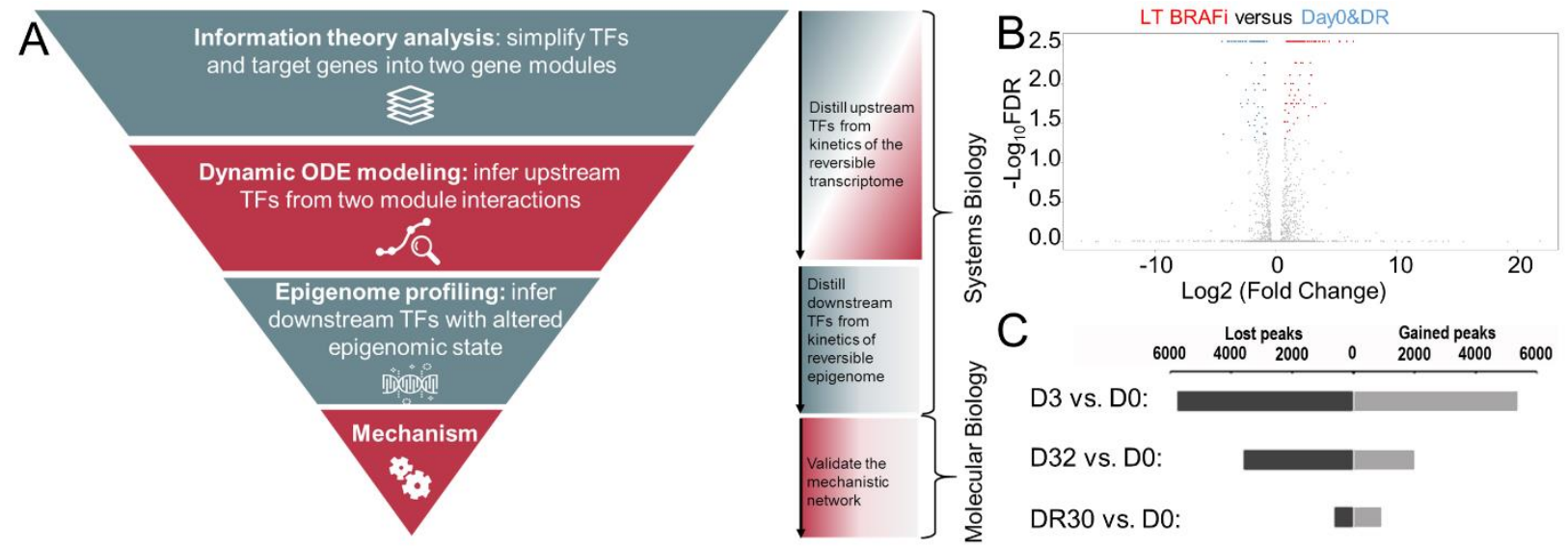

D

$\mathrm{H} 3 \mathrm{~K} 4 \mathrm{me} 3$


Figure S5. Epigenetic reversibility of the adaptive transition. Related to Figure 4. 
(A) Graphical illustration of our methodology that integrates systems biology, bioinformatics and molecular biology approaches for investigating the molecular mechanism of the adaptive resistance.

(B) A volcano plot showing the transcription factors/co-factors that display significant alterations between the drug-resistant state and the drug-sensitive state.

(C) Differential peaks of the ATAC-seq profiles between two different time points. D0, D3, D32, and DR30 denote day-0, day-3, day-32 and drug removal day-30 across the adaptive transition, respectively.

(D) Average H3K4me3 (left) and H3K27ac (right) ChIP-seq signal at the transcription start sites (TSS) across all genes with heatmap representation of each peak shown below. The $\mathrm{X}$-axis shows flanking regions of $+/-3 \mathrm{~kb}$ around the TSS.

(E) ATAC-seq and ChIP-seq profiles at the promoter regions of some representative cell state marker genes, at selected time points across the reversible transition. 
bioRxiv preprint first posted online Aug. 5, 2019; doi: http://dx.doi.org/10.1101/724740. The copyright holder for this preprint (which was not peer-reviewed) is the author/funder, who has granted bioRxiv a license to display the preprint in perpetuity.

All rights reserved. No reuse allowed without permission.

A

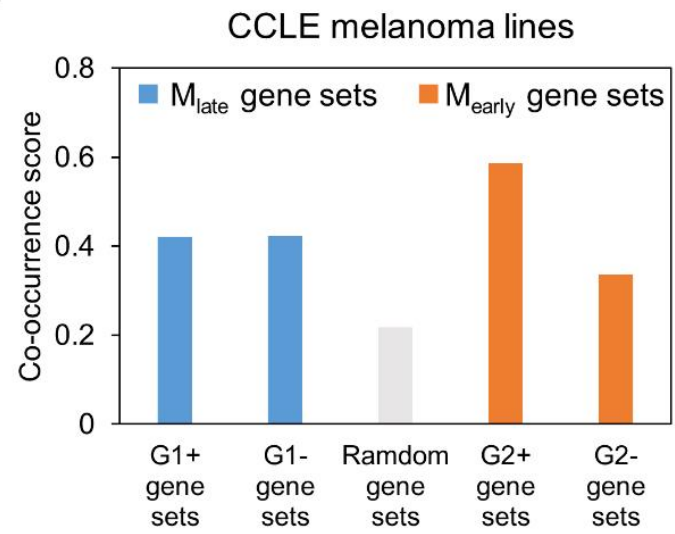

C

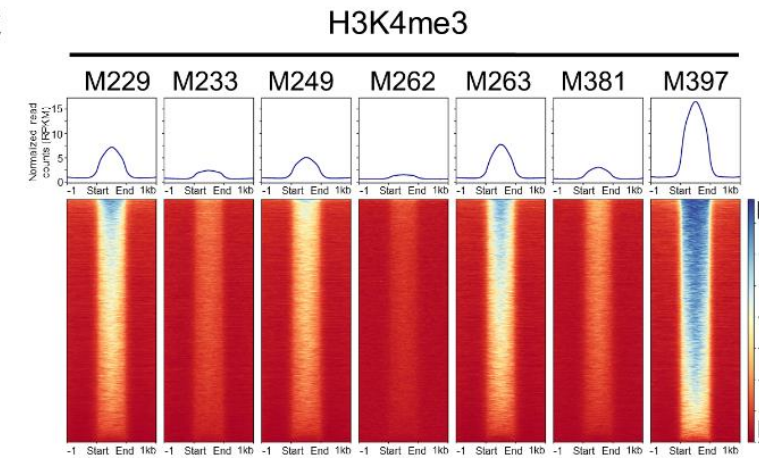

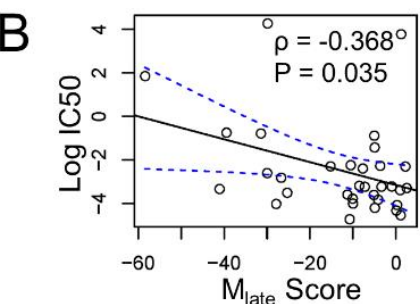
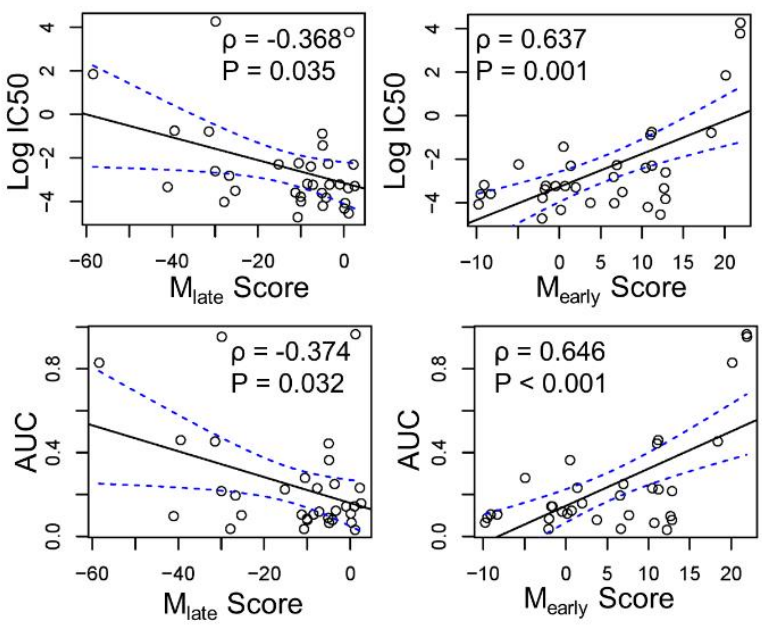

$\mathrm{D}_{30}$

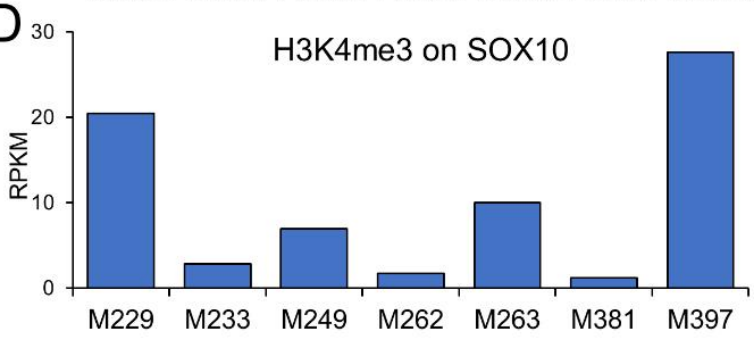

E

M229 M233 M249 M262 M263 M381 M397 CT:
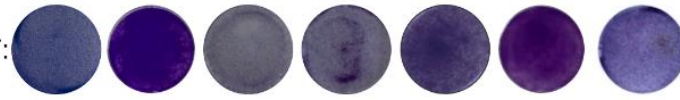

KDM5i:
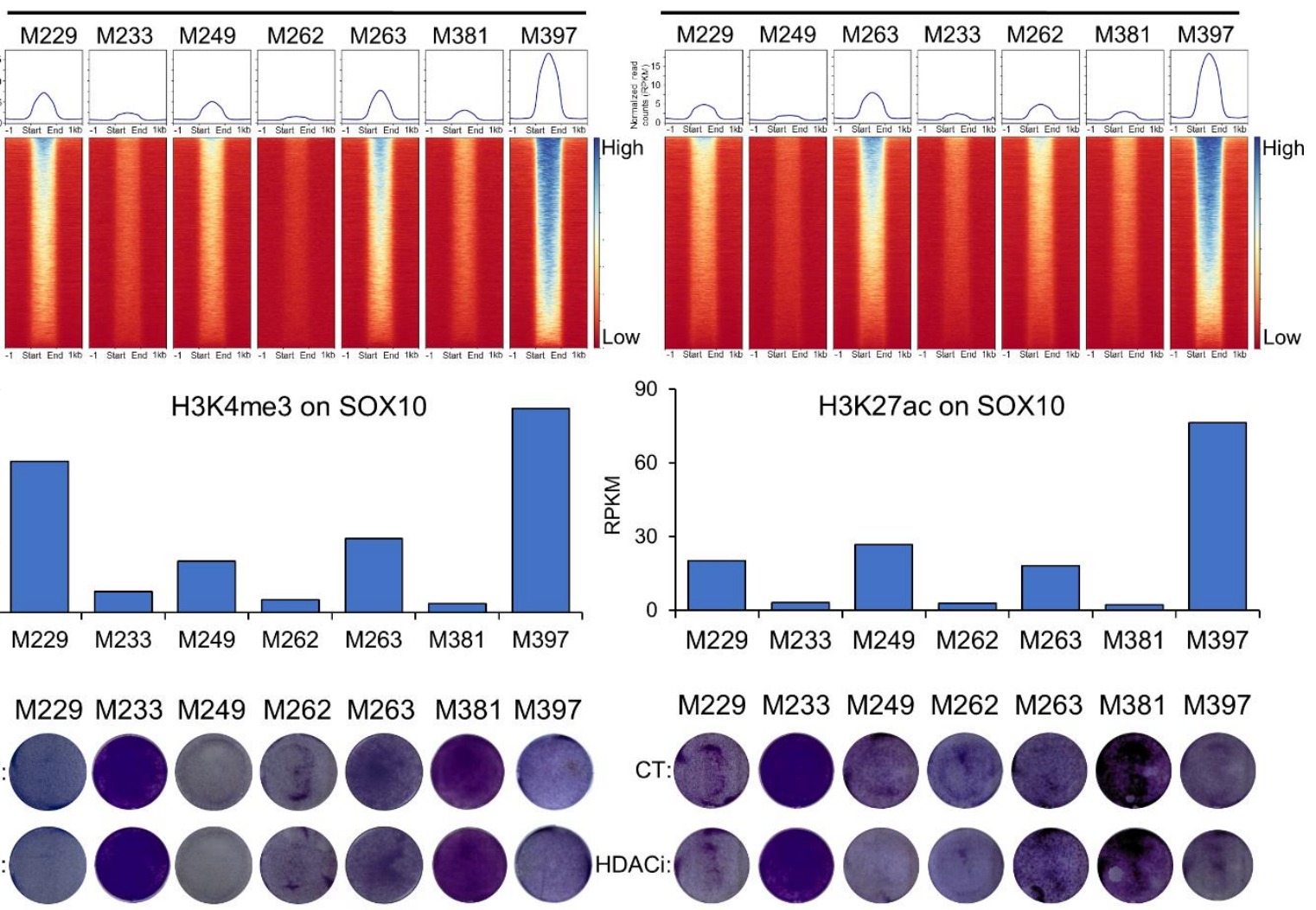

F $\quad$ M229 M233 M249 M262 M263 M381 M397 $\frac{1}{\text { CT CPI }} \frac{1}{\text { CTCPI }} \frac{1}{\text { CT CPI }} \frac{1}{\text { CT CPI }} \frac{1}{\text { CTCPI }} \frac{1}{\text { CT CPI }} \frac{1}{\text { CT CPI }}$ $\mathrm{H} 3 \mathrm{:}=-\infty-\infty-\infty-\infty$ H3K4me3: $--0-0-0-0$

Figure S6. The generality and molecular underpinning of the phenotypic plasticity of patient-derived melanoma cell lines in response to BRAF inhibition. Related to Figure 6. 


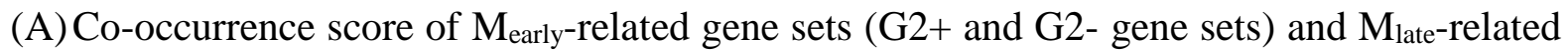
gene sets (G1+ and G1- gene sets) in comparison with randomly selected gene sets calculated from the transcriptome data across all melanoma cells from the CCLE database.

(B) Correlations between $\mathrm{M}_{\text {early }}$ or $\mathrm{M}_{\text {late }}$ module scores and BRAFi resistance levels across $B R A F^{\mathrm{V} 600 \mathrm{E}}$-mutant melanoma cell lines in the GDSC database. The BRAFi resistance levels are quantified by natural $\log$-transformed IC50 $(\mu \mathrm{M})$ values and AUC.

(C) Average H3K4me3 (left) and H3K27ac (right) ChIP-seq signal across promoter regions of all genes for a panel of melanoma cell lines, with heatmap view around TSS shown below. The $\mathrm{x}$-axis shows flanking regions of $+/-1 \mathrm{~kb}$ around each peak center. The $\mathrm{y}$-axis of the top panel represents the read counts normalized by RPKM.

(D) Average H3K4me3 (left) and H3K27ac (right) ChIP-seq signal at the transcription start site (TSS) region of SOX10.

(E) Short-term clonogenic assay of KDM5 inhibitor (left) and HDAC inhibitor (right) across a panel of melanoma cell lines showing no significant toxicity to the cells at the dose used.

(F) Left, western blot of lysates from melanoma either untreated control (CT) or treated with KDM5B inhibitor (CPI) and HDAC inhibitor (Q). H3 is used as loading control. 

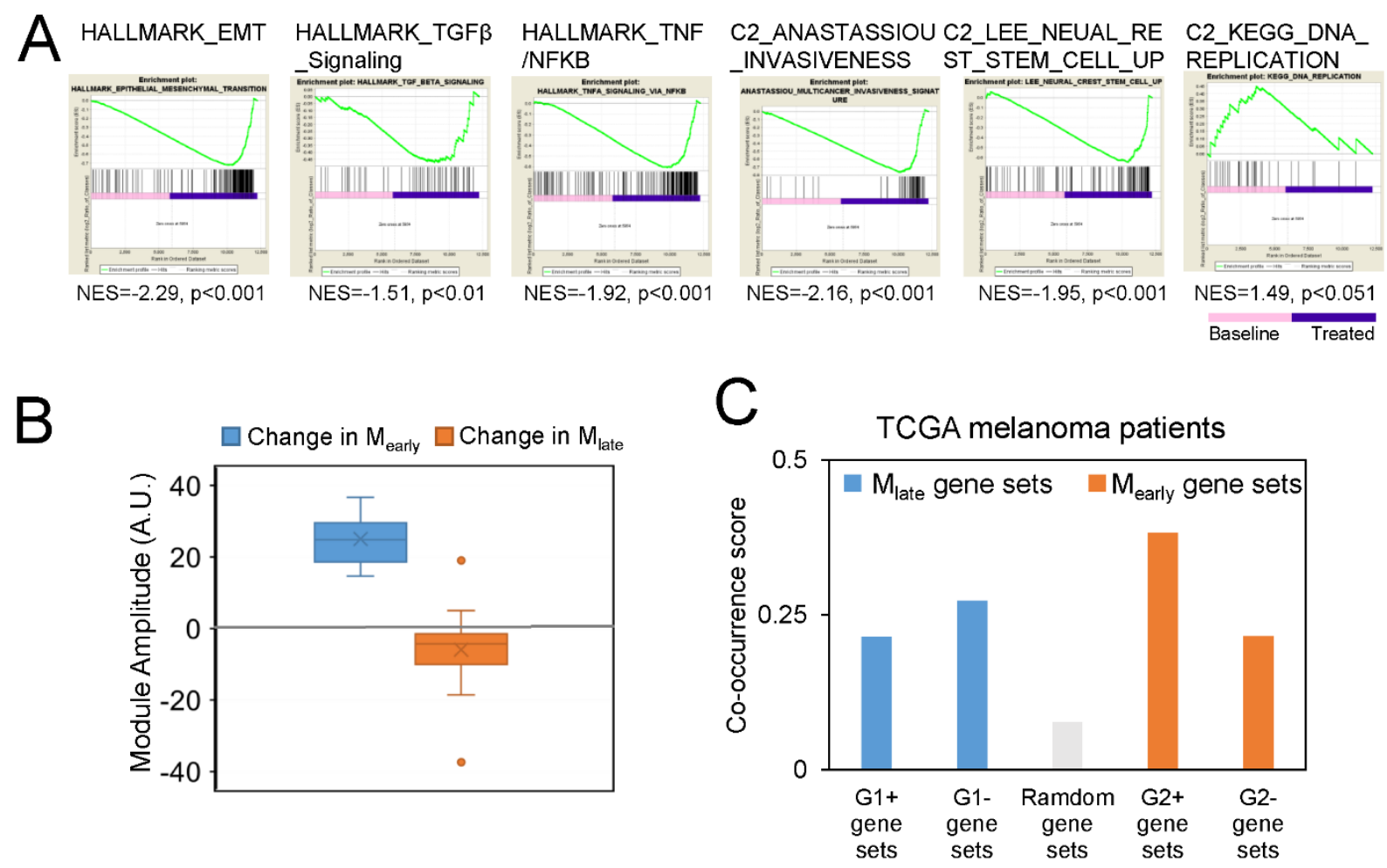

$\mathrm{D}$

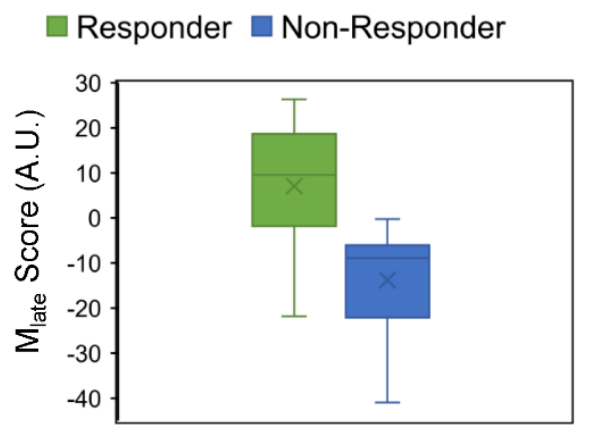

Figure S7. Generality and clinical relevance of adaptive epigenetic mechanism in melanoma patients. Related to Figure 7.

(A) Gene set enrichment analysis (GSEA) on published transcriptome data from a melanoma patient (P2 in Table S6) before and after MAPKi treatment for selected gene sets relevant to the reversible transition observed in our system. NES, normalized enrichment score.

(B) The change in $\mathrm{M}_{\text {early }}$ and $\mathrm{M}_{\text {late }}$ module scores calculated by published transcriptome data from melanoma patients before and after MAPKi treatment.

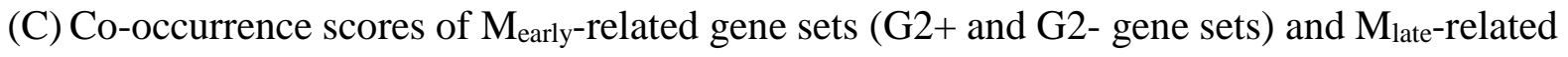
gene sets $(\mathrm{G} 1+$ and $\mathrm{G} 1$ - gene sets) relative to randomly selected gene sets calculated from transcriptome data across all melanoma patients from the TCGA database.

(D) Average $\mathrm{M}_{\text {late }}$ module scores of responders and non-responders to PD-1 checkpoint blockade calculated by published transcriptome data of melanoma patients under PD-1 checkpoint inhibitor treatment (see STAR Methods) 


\section{Supplemental Tables}

Table S1: RNA-seq data (in RPKM) for time-course experiments of M397 and relevant gene module scores from information theory analysis. Data are provided as Excel spreadsheets. 
Table S2. GSEA analysis for selected gene sets between different time points. Statistically significant positive enrichments are highlighted in yellow and negative enrichments in blue.

\begin{tabular}{|c|c|c|c|c|c|c|c|c|c|c|c|c|c|c|c|c|c|c|c|c|c|c|c|c|c|c|}
\hline \multirow[b]{3}{*}{ Gene Set } & \multirow{3}{*}{$\begin{array}{l}\text { MsigDB } \\
\text { Category }\end{array}$} & \multirow{3}{*}{\begin{tabular}{|c|} 
Gene \\
Number
\end{tabular}} & \multicolumn{3}{|c|}{ D3 vs. Do } & \multicolumn{3}{|c|}{ D13 vs. D0 } & \multicolumn{3}{|c|}{ D21 vs. Do } & \multicolumn{3}{|c|}{ D29 vs. D0 } & \multicolumn{3}{|c|}{ D59 vs. DO } & \multicolumn{3}{|c|}{ D21 vs. dr29.D15 } & \multicolumn{3}{|c|}{ dr29.D15 vs. dr29.D30 } & \multicolumn{3}{|c|}{ dr29.D30 vs. D29 } \\
\hline & & & & NOM & FDR & & NOM & FDR & & NOM & FDR & & NOM & & & NOM & FDR & & NOM & & & NOM & & & NOM & FDR \\
\hline & & & NES & p-value & q-value & NES & p-value & q-value & NES & p-value & q-value & NES & p-value & q-value & NES & p-value & q-value & NES & p-value & q-value & NES & p-value & q-value & NES & p-value & q-value \\
\hline Oxidative Phoshorylation & HALLMARK & 198 & -0.84 & 0.854 & 0.856 & -2.22 & 0.000 & 0 & -2.31 & 0.000 & 0.000 & -2.12 & 0.000 & 0.000 & -2.25 & 0.000 & 0.000 & -1.77 & 0.000 & 0.005 & -2.39 & 0.000 & 0.000 & 2.06 & 0.000 & 0.000 \\
\hline Glycolysis & HALLMARK & 198 & & N/A & & & N/A & & 1.28 & 0.034 & 0.187 & 1.45 & 0.007 & 0.083 & 1.16 & 0.123 & 0.316 & & $\mathrm{~N} / \mathrm{A}$ & & 1.4 & 0.009 & 0.104 & -1.24 & 0.065 & 0.261 \\
\hline & & & & 0.056 & 0.500 & -1.79 & 0.000 & 0.002 & -2.49 & 0.000 & 0.000 & -2.65 & 0.000 & 0.000 & -2.65 & 0.000 & 0.000 & -1.79 & 0.000 & 0.003 & -2.66 & 000 & 0.000 & 2.67 & 000 & 0.000 \\
\hline HAN_JNK_Signaling_UP & C2:CGP & 35 & & N/A & & 1.82 & 0.000 & 0.002 & 1.96 & 0.000 & 0.000 & 1.72 & 0.000 & 0.006 & 1.84 & 0.000 & 0.001 & 1.40 & 0.043 & 0.189 & 1.72 & 0.000 & 0.003 & -1.76 & 0.004 & 0.004 \\
\hline PHONG_TNF_Response_via_P & & 222 & & N/A & & 40 & 03 & 0.115 & 1.47 & 00 & & 1.15 & 0.123 & 0.353 & 1.36 & 0.012 & 0.135 & 1.08 & 0.212 & 0.525 & 1.56 & 000 & 0.025 & -1.18 & 090 & 0.327 \\
\hline HINATA_NFKB_Targets_Keratinoyte_Up & C2:CGP & 90 & & N/A & & 1.57 & 0.000 & 0.039 & 1.71 & 0.000 & 0.006 & 1.73 & 0.000 & 0.005 & 1.72 & 0.000 & 0.006 & & N/A & & 2.01 & 0.000 & 0.000 & -1.49 & 0.007 & 0.069 \\
\hline TNFA_Signaling_via_NFKB & HALLMARK & & & N/A & & 1.74 & & 0.006 & 92 & & 0.000 & 1.76 & 0.000 & 0.003 & 1.95 & 0.000 & 0.000 & & $\mathrm{~N} / \mathrm{A}$ & & 1.74 & 000 & 0.006 & -1.55 & 000 & 0.043 \\
\hline WU_Cell_Migration & C2:CGP & 18 & & N/A & & 1.67 & 0.000 & 0.015 & 2.10 & 0.000 & 0.000 & 2.15 & 0.000 & 0.000 & 2.14 & 0.000 & 0.000 & & $\mathrm{~N} / \mathrm{A}$ & & 2.19 & 0.000 & 0.000 & -2.01 & .000 & 0.000 \\
\hline CHUETZ_Breast_Cancer_Ductal_Invasive_Up & & & & $\mathrm{N} / \mathrm{A}$ & & 2.07 & 0.000 & 0 & 2.38 & 0.000 & 0.000 & 2.35 & 0.000 & 0.000 & 2.26 & 0.000 & 0.000 & 1.72 & 0.000 & 0.021 & 2.10 & 000 & 0.000 & 2.33 & .000 & 0.000 \\
\hline LEE Neural Crest Stem Cell Up & C2:CGP & 142 & 1.13 & 0.151 & 0.627 & 1.88 & 0.000 & 0 & 1.89 & 0.000 & 0.000 & 1.66 & 0.000 & 0.013 & 1.46 & 0.007 & 0.076 & 1.68 & 0.000 & 0.032 & 1.57 & 0.000 & 0.024 & -1.68 & 0.000 & 0.012 \\
\hline senymal_T & & 96 & & $\mathrm{~N} / \mathrm{A}$ & & 2.42 & 0.000 & & 2.54 & 0.000 & 0.000 & 2.56 & 0.000 & 0.000 & 2.45 & $0.000>>>2>0$ & 0.000 & 2.02 & 0 & 0.000 & 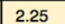 & 000 & 000 & 2.63 & & \\
\hline
\end{tabular}

Table S3. GSEA analysis of the genes associated with $M_{\text {early }}$ (G2) and $M_{\text {late }}$ (G1) gene modules for selected gene sets. Statistically significant positive enrichments are highlighted in yellow and negative enrichments in blue.

\begin{tabular}{|c|c|c|c|c|c|c|c|c|}
\hline \multirow[b]{2}{*}{ Gene Set } & \multirow[b]{2}{*}{$\begin{array}{l}\text { MsigDB } \\
\text { Category }\end{array}$} & \multirow[b]{2}{*}{$\begin{array}{c}\text { Gene } \\
\text { Number }\end{array}$} & \multicolumn{3}{|c|}{ G1 } & \multicolumn{3}{|c|}{ G2 } \\
\hline & & & NES & $\begin{array}{c}\text { NOM } \\
\text { p-value }\end{array}$ & $\begin{array}{c}\text { FDR } \\
\text { q-value }\end{array}$ & NES & $\begin{array}{c}\text { NOM } \\
\text { p-value }\end{array}$ & $\begin{array}{c}\text { FDR } \\
\text { q-value }\end{array}$ \\
\hline Oxidative Phoshorylation & HALLMARK & 198 & 2.26 & 0.000 & 0.000 & -1.51 & 0.005 & 0.082 \\
\hline Glycolysis & HALLMARK & 198 & -1.59 & 0.000 & 0.031 & 1.16 & 0.123 & 0.316 \\
\hline MITF targets* & N/A & 102 & 3.19 & 0.000 & 0.000 & -1.99 & 0.000 & 0.002 \\
\hline HAN_JNK_Signaling_UP & C2:CGP & 35 & -1.45 & 0.002 & 0.085 & 1.42 & 0.047 & 0.183 \\
\hline PHONG_TNF_Response_via_P38_Complete & C2:CGP & 222 & -1.18 & 0.090 & 0.327 & 0.87 & 0.783 & 0.869 \\
\hline HINATA_NFKB_Targets_Keratinoyte_Up & C2:CGP & 90 & -2.12 & 0.000 & 0.000 & & N/A & \\
\hline TNFA_Signaling_via_NFKB & HALLMARK & 198 & -2.31 & 0.000 & 0.000 & & N/A & \\
\hline WU_Cell_Migration & C2:CGP & 181 & -2.33 & 0.000 & 0.000 & & N/A & \\
\hline SCHUETZ_Breast_Cancer_Ductal_Invasive_Up & C2:CGP & 338 & -2.36 & 0.000 & 0.000 & 1.87 & 0.000 & 0.007 \\
\hline LEE_Neural_Crest_Stem_Cell_Up & C2:CGP & 142 & -1.42 & 0.023 & 0.104 & 1.85 & 0.000 & 0.008 \\
\hline Epithelial_Mesenymal_Transition & HALLMARK & 196 & -2.56 & 0.000 & 0.000 & 2.45 & 0.000 & 0.000 \\
\hline
\end{tabular}


Table S4. Fitting parameters used in the ODE modeling.

\begin{tabular}{|c|c|c|c|c|c|c|c|}
\hline & \multicolumn{2}{|c|}{ Input genes for ODE parameter fitting } & $\begin{array}{c}\text { Basal } \\
\text { term }\end{array}$ & \multicolumn{2}{|c|}{$\begin{array}{l}\text { Influence on } \\
\mathrm{M}_{\text {late }}\end{array}$} & \multicolumn{2}{|c|}{$\begin{array}{c}\text { Influence on } \\
\mathrm{M}_{\text {early }}\end{array}$} \\
\hline & $\mathrm{M}_{\text {early }}$ input & $\mathrm{M}_{\text {late }}$ input & $\mathrm{B}_{1} \quad \mathrm{~B}_{\mathrm{e}}$ & $M_{e-1}$ & $M_{1-1}$ & $\mathrm{M}_{1-\mathrm{e}}$ & $M_{e-c}$ \\
\hline \multirow{4}{*}{$\begin{array}{l}\text { Drug } \\
\text { ON }\end{array}$} & $\mathrm{M}_{\text {early_positive_genes }}$ & $\mathrm{M}_{\text {late_positive_genes }}$ & 8.396 .33 & 0.49 & -0.05 & 0.09 & 0.38 \\
\hline & $M_{\text {early_}}$ negative_genes & $\mathrm{M}_{\text {late_positive_genes }}$ & -9.67 .59 & -0.68 & -0.15 & -0.15 & 0.52 \\
\hline & $\mathrm{M}_{\text {early_positive_genes }}$ & $\mathrm{M}_{\text {late_}}$ negative_genes & -3.126 .83 & -0.17 & -0.01 & -0.09 & 0.25 \\
\hline & $M_{\text {early_negative_genes }}$ & $\mathrm{M}_{\text {late_}}$ negative_genes & 2.751 .49 & 0.31 & -0.048 & 0.14 & 0.41 \\
\hline \multirow{4}{*}{$\begin{array}{l}\text { Drug } \\
\text { OFF }\end{array}$} & $\mathrm{M}_{\text {early_}}$ positive_genes & $\mathrm{M}_{\text {late_positive_genes }}$ & 5.475 .35 & 0.38 & 0.16 & -0.12 & 0.53 \\
\hline & $\mathrm{M}_{\text {early-n }}$ negative_genes & $\mathrm{M}_{\text {late_positive_genes }}$ & $-3.46 \quad 6.2$ & -0.18 & 0.03 & -0.05 & 0.22 \\
\hline & $\mathrm{M}_{\text {early }}$ positive_genes & $M_{\text {late- }}$ negative_genes & -1.830 .93 & -0.48 & 0.14 & -0.01 & 0.15 \\
\hline & $M_{\text {early_negative_genes }}$ & $\mathrm{M}_{\text {late}-}$ negative_genes & 10.233 .23 & 0.45 & 0.03 & 0.04 & 0.16 \\
\hline
\end{tabular}


bioRxiv preprint first posted online Aug. 5, 2019; doi: http://dx.doi.org/10.1101/724740. The copyright holder for this preprint (which was not peer-reviewed) is the author/funder, who has granted bioRxiv a license to display the preprint in perpetuity.

All rights reserved. No reuse allowed without permission.

Table S5. The statistical significance of the alterations of chromatin accessibility and histone marks across the adaptive transition. The epigenetic alterations were evaluated in the RelA binding regions of a list of TFs and co-factors that are strongly associated with $\mathrm{M}_{\text {late }}$ and contain RelA binding motifs. The TFs/co-factors that show statistically significant changes across all three epigenome alterations are shown in black.

\begin{tabular}{|c|c|c|c|}
\hline \multirow{2}{*}{ TF name } & H3K4me3 & H3K27ac & ATAC-seq \\
\hline & \multicolumn{3}{|c|}{ P values } \\
\hline SOX10 & $3.2 \times 10^{-11}$ & $1.7 \times 10^{-4}$ & $5.9 \times 10^{-5}$ \\
\hline DNAJC1 & $5.9 \times 10^{-5}$ & $1.2 \times 10^{-3}$ & $9.5 \times 10^{-3}$ \\
\hline $\mathrm{ACTN1}$ & $3.4 \times 10^{-9}$ & $4.0 \times 10^{-5}$ & 0.13 \\
\hline MMS19 & $3.0 \times 10^{-9}$ & $1.2 \times 10^{-4}$ & $6.1 \times 10^{-2}$ \\
\hline REPIN1 & $7.0 \times 10^{-9}$ & $7.3 \times 10^{-4}$ & 0.18 \\
\hline IKZF5 & $7.0 \times 10^{-7}$ & $1.2 \times 10^{-4}$ & 0.15 \\
\hline SIX 4 & $5.1 \times 10^{-8}$ & $6.9 \times 10^{-4}$ & 0.42 \\
\hline VEGFA & $5.8 \times 10^{-7}$ & $5.5 \times 10^{-4}$ & 0.48 \\
\hline KLF10 & $1.1 \times 10^{-6}$ & $8.8 \times 10^{-4}$ & 0.73 \\
\hline E2F3 & $5.4 \times 10^{-6}$ & $1.3 \times 10^{-3}$ & 0.16 \\
\hline HIVEP2 & $2.1 \times 10^{-6}$ & $6.5 \times 10^{-4}$ & 0.95 \\
\hline IRX3 & $1.9 \times 10^{-6}$ & $1.4 \times 10^{-3}$ & 0.60 \\
\hline SGK1 & $4.1 \times 10^{-5}$ & $3.1 \times 10^{-4}$ & 0.14 \\
\hline TADA3 & $1.1 \times 10^{-6}$ & $4.0 \times 10^{-3}$ & 0.97 \\
\hline SERTAD3 & $7.0 \times 10^{-5}$ & $3.8 \times 10^{-4}$ & 0.44 \\
\hline $\mathrm{MAF}$ & $3.1 \times 10^{-5}$ & $5.8 \times 10^{-3}$ & 0.17 \\
\hline SOX5 & $3.1 \times 10^{-6}$ & $1.3 \times 10^{-2}$ & 0.89 \\
\hline PHTF1 & $3.2 \times 10^{-5}$ & $4.2 \times 10^{-3}$ & 0.32 \\
\hline SATB2 & $6.3 \times 10^{-5}$ & $2.6 \times 10^{-3}$ & 0.49 \\
\hline HOXA13 & $5.3 \times 10^{-5}$ & $2.8 \times 10^{-3}$ & 0.68 \\
\hline ZBTB24 & $3.5 \times 10^{-5}$ & $9.4 \times 10^{-3}$ & 0.32 \\
\hline FHIT & $3.5 \times 10^{-5}$ & $8.5 \times 10^{-3}$ & 0.53 \\
\hline IRF1 & $4.9 \times 10^{-5}$ & $1.3 \times 10^{-2}$ & 0.58 \\
\hline ZNF670 & $3.2 \times 10^{-4}$ & $2.6 \times 10^{-3}$ & 0.53 \\
\hline VDR & $9.8 \times 10^{-5}$ & $1.5 \times 10^{-2}$ & 0.79 \\
\hline YAF2 & $9.5 \times 10^{-5}$ & $2.9 \times 10^{-2}$ & 0.47 \\
\hline RNF25 & $4.7 \times 10^{-4}$ & $2.1 \times 10^{-2}$ & 0.67 \\
\hline $\mathrm{ZNF} 280 \mathrm{~B}$ & $1.3 \times 10^{-2}$ & $3.4 \times 10^{-3}$ & 0.55 \\
\hline ETS1 & $1.9 \times 10^{-2}$ & $4.1 \times 10^{-3}$ & 0.36 \\
\hline
\end{tabular}




\begin{tabular}{|c|c|c|c|}
\hline CTDSP1 & $4.5 \times 10^{-3}$ & $1.7 \times 10^{-2}$ & 0.85 \\
\hline TAF10 & $2.0 \times 10^{-2}$ & $7.5 \times 10^{-3}$ & 0.89 \\
\hline RFX2 & $6.1 \times 10^{-3}$ & 0.12 & 0.53 \\
\hline FOXP1 & $4.0 \times 10^{-3}$ & 0.37 & 0.53 \\
\hline STAT1 & $9.4 \times 10^{-2}$ & 0.22 & 0.75 \\
\hline TARBP1 & 0.18 & 0.14 & 0.94 \\
\hline ZNF280A & 0.16 & 0.61 & 0.53 \\
\hline
\end{tabular}

Table S6. Relevant gene expression levels (RPKM) of selected patients before and after the MAPKi treatments from published data. Data are provided as Excel spreadsheets. 